\title{
SYMBOLIC DYNAMICS FOR NONUNIFORMLY HYPERBOLIC SYSTEMS
}

\author{
YURI LIMA
}

\begin{abstract}
This survey describes the recent advances in the construction of Markov partitions for nonuniformly hyperbolic systems. One important feature of this development comes from a finer theory of nonuniformly hyperbolic systems, which we also describe. The Markov partition defines a symbolic extension that is finite-to-one and onto a non-uniformly hyperbolic locus, and this provides dynamical and statistical consequences such as estimates on the number of closed orbits and properties of equilibrium measures. The class of systems includes diffeomorphisms, flows, and maps with singularities.
\end{abstract}

\section{Contents}

Introduction

Acknowledgements

Part 1. Charts, graph transforms, and invariant manifolds

1. Uniformly hyperbolic systems

1.1. Definitions and examples 6

1.2. Preliminaries on the geometry of $M$

1.3. Lyapunov inner product

1.4. Diagonalization of derivative 11

1.5. Lyapunov charts, change of coordinates

1.6. Graph transforms: construction of invariant manifolds

1.7. Higher dimensions

2. Nonuniformly hyperbolic systems

2.1. Definitions and examples 17

2.2. The nonuniformly hyperbolic locus $\mathrm{NUH}_{\chi}$

2.3. Diagonalization of derivative 20

2.4. Pesin charts, the parameter $Q(x)$, change of coordinates 20

2.5. Temperedness and the parameter $q(x)$ 21

2.6. Sizes of invariant manifolds: the parameters $q^{s}(x), q^{u}(x) \quad 22$

2.7. Graph transforms: construction of invariant manifolds 23

2.8. Higher dimensions 24

3. Maps with discontinuities and bounded derivative 25

3.1. Definitions and examples 26

Date: June 16, 2020.

1991 Mathematics Subject Classification. 37-02, 37B10, 37C05, 37C35, 37C83, 37D25, 37D35.

Key words and phrases. Markov partition, Pesin theory, symbolic dynamics.

The author is supported by CNPq and Instituto Serrapilheira, grant "Jangada Dinâmica: Impulsionando Sistemas Dinâmicos na Região Nordeste". 
3.2. Nonuniform hyperbolicity 26

4. Maps with discontinuities and unbounded derivative 28

4.1. Definitions and examples [28

4.2. Nonuniform hyperbolicity 29

Part 2. Symbolic dynamics 30

5. Symbolic dynamics for uniformly hyperbolic systems 32

5.1. Markov partitions/sections 33

5.2. Markov partitions/sections generate symbolic models 35

5.3. Markov partitions for two-dimensional hyperbolic toral automorphisms 355

5.4. The method of successive approximations for diffeomorphisms 36

5.5. The method of successive approximations for flows

5.6. The method of pseudo-orbits 39

6. Symbolic dynamics for nonuniformly hyperbolic systems 44

6.1. Preliminaries 45

6.2. Coarse graining 48

6.3. Improvement lemma 49

6.4. Inverse theorem 50

6.5. Bowen-Sinaŭ refinement 51

6.6. Affiliation and Bowen relation 53

6.7. Higher dimensions 54

6.8. Uniform hyperbolicity versus nonuniform hyperbolicity 54

6.9. Surface maps with discontinuities

Part 3. Applications

7. Estimates on the number of closed orbits

8. Equilibrium measures 60

References 62

\section{INTRODUCTION}

Markov partitions are a powerful tool in the modern theories of dynamical systems and ergodic theory. They were introduced to these fields at the end of the sixties, see the foundational works of Adler \& Weiss and Sinar [AW67, AW70, Sin68b, Sin68a and references therein, and have played a crucial role ever since. Roughly speaking, a Markov partition is a partitioning of the phase space of a system into pieces, that allows to represent trajectories by paths on a graph. The dynamics of paths on a graph is much simpler to understand, and many of its statistical properties can therefore be pushed to the original dynamical system. This approach was extensively developed in the late sixties and early seventies to uniformly hyperbolic systems, and its consequences include many breakthroughs in smooth ergodic theory. The method developed by Bowen Bow08 will be of particular importance to us: locally representing the dynamics as a small perturbation of a hyperbolic matrix, he used the theory of pseudo-orbits used by Anosov Ano70 and by himself Bow70b, Bow71 Bow72b to obtain a Markov cover and then refine it to a Markov partition. Due to uniform hyperbolicity, the Markov partitions are finite. 
Following Bowen's philosophy, Katok showed that a hyperbolic ergodic measure that is invariant under a $C^{1+\beta}$ diffeomorphism has horseshoes approximating its entropy. A measure is hyperbolic if its Lyapunov exponents are non-zero, and this introduces the concept of nonuniform hyperbolicity: the hyperbolicity is not necessarily observed at every iteration but only on the average. In the late seventies, Pesin developed a global theory to treat $C^{1+\beta}$ nonuniformly hyperbolic systems Pes76, Pes77a, Pes77b], nowadays known as Pesin theory. See the book BP07]. Pesin's idea was to construct local charts, nowadays called Pesin charts, to represent the dynamics of a nonuniformly hyperbolic diffeomorphism again as a small perturbation of a hyperbolic matrix. The difference from the uniformly hyperbolic situation is that the domain of the Pesin chart is no longer uniform in size and depends on the quality of hyperbolicity at the point. In [Kat80], Katok combined Pesin theory with a fine theory of pseudo-orbits and, to avoid the possible degeneracy of Pesin charts, restricted the analysis to Pesin blocks, which are non-invariant subsets of the phase space where nonuniform hyperbolicity is essentially uniform. For details, see the supplementary chapter by Katok \& Mendoza KH95. Since a horseshoe naturally carries a Markov partition, Katok's result can be seen as the construction of finite Markov partitions that approximate the topological entropy. The applications using the now called Katok horseshoes are countless. Nevertheless, this approach is not genuinely nonuniformly hyperbolic, since it does not treat at once regions where the degeneracy of Pesin charts occurs. In other words, a single Pesin block does not encompass the whole dynamics (for instance, it usually does not have full topological entropy).

This difficulty stood unsolved for many years, until Sarig recently bypassed it, constructing countable Markov partitions with full topological entropy for $C^{1+\beta}$ surface diffeomorphisms [Sar13]. His methods are more suitable for adaptations and generalizations, and are now being further refined to settings in which the previous theory was not able to reach, such as billiard maps. Here are some of the developments:

- Lima and Sarig for three dimensional flows without fixed points LS19].

- Lima and Matheus for surface maps with discontinuities [LM18].

- Ben Ovadia for diffeomorphisms in any dimension BO18.

Now, the Markov partitions are countable. This is unavoidable to treat the regions where the Pesin charts degenerate. Not only these latter results are stronger than the previous ones in the literature, they also cover much broader classes of examples such as geodesic flows on surfaces with nonpositive curvature and Bunimovich stadia. Using them, many dynamical and statistical properties were established: counting on the number of closed orbits Sar13, LS19, LM18, BD20, BO18, Buz20, counting on the number of measures of maximal entropy [Sar13. LS19, BO18, BCS18, ergodic properties of equilibrium measures [Sar11, LLS16], the almost Borel structure of surface diffeomorphisms [BB17, and the generic simplicity of Lyapunov spectrum of nonuniformly hyperbolic diffeomorphisms [BPVL19].

Generally speaking, countable Markov partitions are indeed necessary to code nonuniformly hyperbolic systems: while the set of topological entropies associated to finite Markov partitions is countable, the set of topological entropies of nonuniformly hyperbolic systems is $[0, \infty)$. This occurs e.g. among $C^{\infty}$ diffeomorphisms in surfaces, where the topological entropy is continuous [New89, Thm. 6]. For 
instance, consider the two-dimensional disc: the identity map has zero entropy, and Smale's horseshoe has topological entropy equal to $\log 2$ (see Section 1.1 for details on this latter example). Since these maps are homotopic, the set of values for the topological entropy contains $[0, \log 2]$ and thus, taking powers, it is equal to $[0, \infty)$. The same occurs for $C^{\infty}$ diffeomorphisms in the two-dimensional torus: in the one-parameter family of standard maps $f_{k}(x, y)=(-y+2 x+k \sin (2 \pi x), x)$, the topological entropy reaches arbitrarily large values Dua94.

As already mentioned, Markov partitions provide many dynamical and statistical consequences because the dynamics of paths on a graph is simple to understand. In general, any partition generates a symbolic representation of the system, given by the shift map acting on a subset of the symbolic space of paths on a graph. For Markov partitions, such symbolic representation is defined not only on a subset but on the whole space of paths on the graph. This is already a big advantage, but for effectiveness of applications it is important to understand the coding map, that relates real trajectories to paths on the graph. If, for instance, the coding map is finite-to-one (i.e. every point has finitely many pre-images) then measures on the original system are related to measures on the symbolic space, and the relation preserves entropy (by the Abramov-Rokhlin formula). This happens for uniformly hyperbolic systems almost automatically, but constitutes a major difficulty for nonuniformly hyperbolic ones. Indeed, all previous attempts before Sarig failed exactly at this point. Sarig did not prove that the coding map is finite-to-one, but that it is morally finite-to-one: after passing to recurrent subsets (defined by some recurrence assumptions), the coding map is finite-to-one. This was the motivation to perform Pesin theory in a much finer way, which has a central importance in the recent constructions of Markov partitions. Having this in mind, this survey has two main goals:

- Discuss the theory of nonuniformly hyperbolic systems.

- Use this theory to construct countable Markov partitions that generate finite-toone coding maps.

Since the main reason to construct Markov partitions and finite-to-one codings is to understand dynamical and statistical properties of smooth dynamical system, we also provide applications in this context.

Two words of caution. Firstly, we do not provide a historical account on the developments of Markov partitions. Secondly, we do not discuss symbolic dynamics in great extent, but only finite-to-one codings for systems with uniform and nonuniform hyperbolicity. Away from these contexts, there are various tools in symbolic dynamics that are important on their own and provide far reaching conclusions, such as Milnor-Thurston's theory of kneading sequences [MT88], Hofbauer towers [Tak73, Hof78, Hof79 Hof81, symbolic extensions [BD04, Bur11, Dow11], Yoccoz puzzles [Yoc15], Young towers YYou98], and more.

We divide the survey into three parts. In Part 1, we discuss the theory of invariant manifolds for uniformly and nonuniformly hyperbolic systems, including the construction of local charts and graph transforms. For simplicity of exposition, most of the arguments will be discussed in dimension two, both for diffeomorphisms and maps with discontinuities, but we also sketch how to make the constructions in higher dimension. In Part 2, we extend this theory to pseudo-orbits, and explain 
how to use them to construct Markov partitions and finite-to-one coding maps. In Part 3, we provide applications.

\section{ACKNOWLEDGEMENTS}

These notes grew up from a series of minicourses the author has given in the past three years, in various institutions: ICERM (Providence, USA), Jagiellonian University (Krakow, Poland), ICTP (Trieste, Italy), Brigham Young University (Provo, USA), PUC-Chile (Santiago, Chile), University of Warwick (Coventry, UK), and Tsinghua University (Beijing, China). The author would like to thank the institutions and local organizers for the invitations, and Mark Demers, Gerhard Knieper for valuable suggestions. The first version of this document was prepared while the author was supported by $\mathrm{CNPq}$ and by Instituto Serrapilheira, grant Serra-1709-20498.

\section{Part 1. Charts, graph transforms, and invariant manifolds}

We introduce tools that allow to pass from the infinitesimal information given by the assumption on the derivative of the system to a representation of its local dynamics. The main goal is to introduce three tools:

- Local charts, which locally represent the dynamics as a small perturbation of a hyperbolic matrix.

- Graph transforms, which explore the hyperbolic feature of the local representation to identify points that remain close to trajectories.

- Invariant manifolds, which provide dynamical coordinates and allow to separate the future and past behavior of the system.

For methodological reasons, the discussion is divided into sections, each of them treating a different class of systems. In Section 1 we deal with uniformly hyperbolic diffeomorphisms. In Section 2 we consider nonuniformly hyperbolic diffeomorphisms. In the last two sections, we discuss nonuniformly hyperbolic surface maps with discontinuities: in Section 3 we assume bounded derivative (e.g. Poincaré return maps of flows without fixed points), and in Section 4 we allow the derivative to grow polynomially fast to infinity (e.g. billiard maps). The discussion in each new section emphasizes the new input that is necessary to make the construction work, so we recommend the reader to follow the text as presented here.

\section{UNIFORMLY HYPERBOLIC SYSTEMS}

Uniformly hyperbolic systems are at the heart of the great developments that tailored the beginning of the modern theories of dynamical systems and ergodic theory, and constitute one of the nicest situations in which a system shows chaos in almost any sense of the word: exponential divergence of the trajectories, denseness of periodic orbits, among others. The study of uniformly hyperbolic systems now has a long history, that began already in the 19th century with the study of geodesic flows on surfaces of constant negative curvature by Hadamard Had98. This topic was extensively developed between 1920 and 1940, among which we mention the work of Morse [Mor34], Hedlund Hed39], and Hopf Hop39. Hop40. About 1940, it became clear that geodesic flows were a particular case of the real setup of interest, and Anosov realized that the theory goes through under a more general condition, 
that he called the (U)-condition. In his own words, a system satisfies the (U)condition if it has "exponential dichotomy of solutions" [Ano69, pp. 22]. Anosov made fundamental contributions to the study of (U)-systems, including their ergodicity [Ano69, Thm. 4]. Nowadays, (U)-systems are called Anosov systems, and the assumption on exponential dichotomy of solutions is called hyperbolicity.

While the Russian school focused on the probabilistic aspects of dynamical systems, the American school led by Smale focused on the topological aspects. Smale discovered the horseshoe, which is the first example of a system shown to have infinitely many periodic points and yet being structurally stable. The history of the discovery is explained in [Sma98, where Smale claims that "the horseshoe is a natural consequence of a geometrical way of looking at the equations of CartwrightLittlewood and Levinson". A horseshoe has similar properties to Anosov systems, because the recurrent (but not all) trajectories are hyperbolic. For the purpose of dynamics, this is satisfactory because a non-recurrent trajectory is uninteresting for dynamical purposes. Having this in mind, Smale introduced the notion of Axiom A systems, where hyperbolicity is required to hold only on the non-wandering set. For transitive Anosov systems, the notions of Anosov and Smale coincide, but there are Axiom A systems that are not Anosov. What we call uniformly hyperbolic are Anosov and Axiom A systems. Nowadays there are great textbooks describing such systems, see e.g. BS02, Shu87, KH95.

The main result of this section is the existence of local invariant manifolds. It holds for $C^{1}$ uniformly hyperbolic systems, see e.g. [Shu87], but to keep an analogy with the nonuniformly hyperbolic context to be discussed in Section 2, we will assume for most of the time that the system is $C^{1+\beta}$, see definition in Section 1.2 .

1.1. Definitions and examples. Let $M$ be a closed (compact without boundary) connected smooth Riemannian manifold, and let $f: M \rightarrow M$ be a $C^{1}$ diffeomorphism.

Anosov Diffeomorphism: We call $f$ an Anosov diffeomorphism if there is a continuous splitting $T M=E^{s} \oplus E^{u}$ and constants $C>0, \kappa<1$ s.t.:

(1) INVARIANCE: $d f\left(E_{x}^{s / u}\right)=E_{f(x)}^{s / u}$ for all $x \in M$.

(2) Contraction:

- Vectors in $E^{s}$ contract in the future: $\left\|d f^{n} v\right\| \leq C \kappa^{n}\|v\|$ for all $v \in E^{s}, n \geq 0$.

- Vectors in $E^{u}$ contract in the past: $\left\|d f^{-n} v\right\| \leq C \kappa^{n}\|v\|$ for all $v \in E^{u}, n \geq 0$.

A closed $f$-invariant set $\Lambda$ satisfying the above properties is called uniformly hyperbolic or simply hyperbolic, hence a diffeomorphism is Anosov if the whole phase space $M$ is hyperbolic. The continuity condition of the splitting in the definition indeed follows from the other assumptions, see e.g. [BS02, Proposition 5.2.1]. As a matter of fact, the splitting is Hölder continuous, as proved by Anosov [Ano67], see also the appendix of Bal95 for a simpler proof. Condition (2) is the exponential dichotomy of solutions mentioned by Anosov. Usually, the above assumptions are rather restrictive because they require the properties on all of $M$, and sometimes parts of $M$ are not dynamically relevant. The set where interesting dynamics can occur is called the non-wandering set. 
NON-WANDERING SET $\Omega(f)$ : The non-wandering set of $f$, denoted by $\Omega(f)$, is the set of all $x \in M$ s.t. for every neighborhood $U \ni x$ there exists $n \neq 0$ s.t. $f^{n}(U) \cap U \neq \varnothing$.

In other words, a point is non-wandering if, no matter how small we choose a neighborhood, it does self-intersect in the future or in the past. In particular, every periodic point is non-wandering. Let $\operatorname{Per}(f)$ denote the set of periodic points of $f$.

Axiom A diffeomorphism: We call $f$ an Axiom A diffeomorphism if:

(1) Denseness of Periodic orbits: $\overline{\operatorname{Per}(f)}=\Omega(f)$.

(2) Hyperbolicity: $\Omega(f)$ is hyperbolic, i.e. there exists a continuous splitting $T_{\Omega(f)} M=E^{s} \oplus E^{u}$ and constants $C>0, \kappa<1$ s.t.:

○ $d f\left(E_{x}^{s / u}\right)=E_{f(x)}^{s / u}$ for all $x \in \Omega(f)$.

- $\left\|d f^{n} v\right\| \leq C \kappa^{n}\|v\|$ for all $v \in E^{s}, n \geq 0$.

○ $\left\|d f^{-n} v\right\| \leq C \kappa^{n}\|v\|$ for all $v \in E^{u}, n \geq 0$.

Every Anosov diffeomorphism is Axiom A, but the converse is false. Now let $\varphi: M \rightarrow M$ be a flow generated by a vector field $X$ of class $C^{1}$. The definitions of uniformly hyperbolic flows are similar to the ones above, having in mind that in the flow direction there is no contraction nor expansion. Below, $\langle X\rangle$ represents the subbundle generated by $X$, whose vector space at $x$ is the line generated by $X_{x}$.

Anosov flow: We call $\varphi$ an Anosov flow if $X \neq 0$ everywhere and if there is a continuous splitting $T M=E^{s} \oplus\langle X\rangle \oplus E^{u}$ and constants $C>0, \kappa<1$ s.t.:

(1) INVARIANCE: $d \varphi^{t}\left(E_{x}^{s / u}\right)=E_{\varphi^{t}(x)}^{s / u}$ for all $x \in M, t \in \mathbb{R}$.

(2) Contraction:

- Vectors in $E^{s}$ contract in the future: $\left\|d \varphi^{t} v\right\| \leq C \kappa^{t}\|v\|$ for all $v \in E^{s}, t \geq 0$.

- Vectors in $E^{u}$ contract in the past: $\left\|d \varphi^{-t} v\right\| \leq C \kappa^{t}\|v\|$ for all $v \in E^{u}, t \geq 0$.

Similarly, a closed $f$-invariant set $\Lambda$ satisfying the above properties is called uniformly hyperbolic or simply hyperbolic.

NON-WANDERING SET $\Omega(\varphi)$ : The non-wandering set of $\varphi$, denoted by $\Omega(\varphi)$, is the set of all $x \in M$ s.t. for every neighborhood $U \ni x$ and for every $t>0$ there exists $T \in \mathbb{R}$ with $|T|>t$ s.t. $\varphi^{T}(U) \cap U \neq \varnothing$.

The above definition is natural, since $\varphi^{t}(U) \cap U \neq \varnothing$ for any $t$ sufficiently small. Let $\operatorname{Per}(\varphi)$ denote the set of periodic points of $\varphi$.

Axiom A flow: We call $\varphi$ an Axiom A flow if $X \neq 0$ on $\Omega(\varphi)$ and:

(1) Denseness of PeRiodic orbits: $\overline{\operatorname{Per}(\varphi)}=\Omega(\varphi)$.

(2) Hyperbolicity: $\Omega(\varphi)$ is hyperbolic, i.e. there exists a continuous splitting $T_{\Omega(f)} M=E^{s} \oplus\langle X\rangle \oplus E^{u}$ and constants $C>0, \kappa<1$ s.t.:

$\circ d \varphi^{t}\left(E_{x}^{s / u}\right)=E_{\varphi(x)}^{s / u}$ for all $x \in \Omega(f), t \in \mathbb{R}$.

- $\left\|d \varphi^{t} v\right\| \leq C \kappa^{t}\|v\|$ for all $v \in E^{s}, t \geq 0$.

- $\left\|d \varphi^{-t} v\right\| \leq C \kappa^{t}\|v\|$ for all $v \in E^{u}, t \geq 0$.

We call a system uniformly hyperbolic if it is either Anosov or Axiom A. Here are three classical examples. 
1. Every hyperbolic matrix 1$]$ $f=f_{A}: \mathbb{T}^{n} \rightarrow \mathbb{T}^{n}$ on the $n$-dimensional torus $\mathbb{T}^{n}=\mathbb{R}^{n} / \mathbb{Z}^{n}$. For $A=\left[\begin{array}{ll}2 & 1 \\ 1 & 1\end{array}\right]$, the Anosov diffeomorphism $f$ is known as Arnold's cat map or simply cat map. Although the dynamics of $A$ is simple, the dynamics of $f$ as seen on the canonical fundamental domain $[0,1]^{2}$ of $\mathbb{T}^{2}$ is rather complicated, see Figure 1 . See also



Figure 1. Arnold's cat map.

BS02, §1.7].

2. Smale's horseshoe, generated by the geometrical configuration in Figure 2 below. See [Shu87, BS02].

\begin{tabular}{|l|}
\hline $\mathrm{A}$ \\
\hline $\mathrm{B}$ \\
\hline $\mathrm{C}$ \\
\hline
\end{tabular}
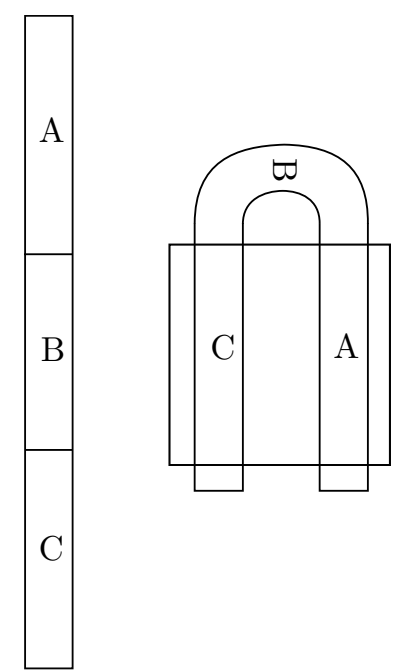

Figure 2. The geometrical mechanism in the creation of a horseshoe.

\footnotetext{
${ }^{1} \mathrm{~A}$ matrix is hyperbolic if none of its eigenvalues lie on the unit circle.
} 
3. The geodesic flow on a closed manifold with negative sectional curvature is Anosov, see Figure 3. Its hyperbolicity is more complicated to describe, since it is defined on the (unit) tangent bundle of the manifold and its derivative in the tangent bundle of this (unit) tangent bundle. We refer the reader to BP13, Chapter 1] for a discussion on the two-dimensional case with constant curvature, to Ebe01 for a more general discussion, and to Kni02, Section 1.3] for a proof of hyperbolicity.

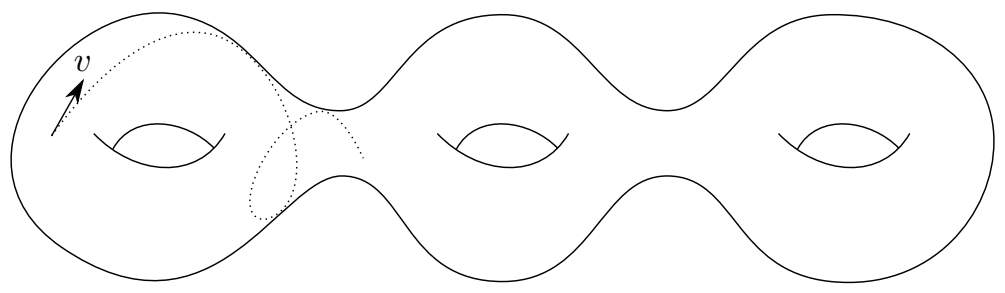

Figure 3. A surface with negative curvature, whose geodesic flow is Anosov. Attention: the figure is somewhat misleading because it induces to think that the curvature in the extreme left and right hand sides is positive.

1.2. Preliminaries on the geometry of $M$. It is easy to define Hölder continuity for maps on euclidean spaces. For instance, $f: U \subset \mathbb{R}^{n} \rightarrow \mathbb{R}^{m}$ is $\beta$-Hölder if there is $\mathfrak{K}>0$ s.t. $\|f(x)-f(x)\| \leq \mathfrak{K}\|x-y\|^{\beta}$ for all $x, y \in U$. Similarly, $f$ is $C^{1+\beta}$ if it is $C^{1}$ and there is $\mathfrak{K}>0$ s.t. $\left\|d f_{x}^{ \pm 1}-d f_{y}^{ \pm 1}\right\| \leq \mathfrak{K}\|x-y\|^{\beta}$ for all $x, y \in U$. For general manifolds, this is slightly more complicated because the derivatives are defined in different tangent spaces and so we need to compare the geometry of nearby tangent spaces. For that we use the local charts provided by the exponential maps, which are intrinsic of the geometry of $M$. For the inexperienced reader, we suggest to make the calculations in the euclidean situation, where all exponential maps are identity.

We are assuming $M$ is a closed connected smooth Riemannian manifold. We denote open balls in $M$ by $B(x, r)$. Given $r>0$, let $B_{x}[r] \subset T_{x} M$ be the open ball with center 0 and radius $r$. For each $x \in M$, let $\exp _{x}: T_{x} M \rightarrow M$ be the exponential map at $x$, i.e. $\exp _{x}(v)=\gamma(1)$ where $\gamma$ is the unique geodesic s.t. $\gamma^{\prime}(0)=v$. Given $x \in M$, let $\operatorname{inj}(x)$ be the radius of injectivity at $x$, i.e. $\operatorname{inj}(x)$ is the largest $r>0$ s.t. the restriction of $\exp _{x}$ to $B_{x}[r]$ is a diffeomorphism onto its image. Choose $\mathfrak{r}_{0}>0$ s.t. for $D_{x}:=B\left(x, 2 \mathfrak{r}_{0}\right)$ the following holds:

○ $\exp _{x}: B_{x}\left[2 \mathfrak{r}_{0}\right] \rightarrow M$ is a 2-bi-Lipschitz diffeomorphism onto its image.

○ If $y \in D_{x}$ then $\operatorname{inj}(y) \geq 2 \mathfrak{r}_{0}$ and $\exp _{y}^{-1}: D_{x} \rightarrow T_{y} M$ is a 2 -bi-Lipschitz diffeomorphism onto its image.

Such $\mathfrak{r}_{0}>0$ exists because $d\left(\exp _{x}\right)_{0}=\operatorname{Id}$ and $M$ is compact.

For $x, x^{\prime} \in M$, let $\mathscr{L}_{x, x^{\prime}}:=\left\{A: T_{x} M \rightarrow T_{x^{\prime}} M: A\right.$ is linear $\}$ and $\mathscr{L}_{x}:=\mathscr{L}_{x, x}$. If $d(x, y)<\operatorname{inj}(x)$, then there is a unique radial geodesic $\gamma$ joining $x$ to $y$, and the parallel transport $P_{x, y}$ along this geodesic is in $\mathscr{L}_{x, y}$. Let $x, x^{\prime} \in M$ and $y, z \in M$ s.t. $d(x, y)<\operatorname{inj}(x)$ and $d\left(x^{\prime}, z\right)<\operatorname{inj}\left(x^{\prime}\right)$. Given $A \in \mathscr{L}_{y, z}$, let $\widetilde{A} \in \mathscr{L}_{x, x^{\prime}}, \widetilde{A}:=$ $P_{z, x^{\prime}} \circ A \circ P_{x, y}$. By definition, $\widetilde{A}$ depends on $x, x^{\prime}$ but different base points define a map that differs from $\widetilde{A}$ by pre and post composition with isometries. In particular, 
$\|\widetilde{A}\|$ does not depend on the choice of $x, x^{\prime}$. Similarly, if $A_{i} \in \mathscr{L}_{y_{i}, z_{i}}$ then $\left\|\widetilde{A_{1}}-\widetilde{A_{2}}\right\|$ does not depend on the choice of $x, x^{\prime}$. With this notation, we say that $f$ is a $C^{1+\beta}$ diffeomorphism if $f \in C^{1}$ and there exists $\mathfrak{K}>0$ s.t.:

- If $y_{1}, y_{2} \in D_{x}$ and $f\left(y_{1}\right), f\left(y_{2}\right) \in D_{x^{\prime}}$ then $\left\|\widetilde{d f_{y_{1}}}-\widetilde{d f_{y_{2}}}\right\| \leq \mathfrak{K} d\left(y_{1}, y_{2}\right)^{\beta}$. ○ If $y_{1}, y_{2} \in D_{x}$ and $f^{-1}\left(y_{1}\right), f^{-1}\left(y_{2}\right) \in D_{x^{\prime \prime}}$ then $\left\|\widetilde{d f_{y_{1}}^{-1}}-\widetilde{d f_{y_{2}}^{-1}}\right\| \leq \mathfrak{K} d\left(y_{1}, y_{2}\right)^{\beta}$.

1.3. Lyapunov inner product. By the definitions in Subsection 1.1. hyperbolicity implies that the restriction of $d f$ to $E^{s}$ is eventually a contraction, exactly when $n$ is large enough so that $C \kappa^{n}<1$, and the same occurs to the restriction of $d f^{-1}$ to $E^{u}$. It turns out that we can define a new inner product, equivalent to the original, for which $d f \uparrow_{E^{s}}$ and $d f^{-1} \uparrow_{E^{u}}$ are contractions already since the first iterate. Such inner product is known as adapted metric or Lyapunov inner product. For consistency with the nonuniformly hyperbolic situation, we will use the later notation. The idea of changing an eventual contraction to a contraction is a popular trick in dynamics, and it appears in various contexts, from Picard's theorem on existence and uniqueness of solutions of ordinary differential equations to the construction of invariant manifolds, as we will see here. There are many different ways of defining such inner product, see e.g. [Shu87, Proposition 4.2]. Here, we follow an approach similar to BS02, Proposition 5.2.2].

We assume that $f: M \rightarrow M$ is a uniformly hyperbolic diffeomorphism, and we let $\langle\cdot, \cdot\rangle$ be the Riemannian metric on $M$. For simplicity of notation, we assume that $f$ is Anosov, with invariant splitting $T M=E^{s} \oplus E^{u}$ (for Axiom A, the definitions are made inside $\Omega(f))$. Fix $\kappa<\lambda<1$.

LYAPUNOV INNER PRODUCT: We define an inner product $\langle\langle\cdot, \cdot\rangle\rangle$ on $M$, called Lyapunov inner product, by the following identities:

○ For $v_{1}^{s}, v_{2}^{s} \in E^{s}$ :

$$
\left\langle\left\langle v_{1}^{s}, v_{2}^{s}\right\rangle\right\rangle=2 \sum_{n \geq 0} \lambda^{-2 n}\left\langle d f^{n} v_{1}^{s}, d f^{n} v_{2}^{s}\right\rangle
$$

○ For $v_{1}^{u}, v_{2}^{u} \in E^{u}$ :

$$
\left\langle\left\langle v_{1}^{u}, v_{2}^{u}\right\rangle\right\rangle=2 \sum_{n \geq 0} \lambda^{-2 n}\left\langle d f^{-n} v_{1}^{u}, d f^{-n} v_{2}^{u}\right\rangle .
$$

- For $v^{s} \in E^{s}$ and $v^{u} \in E^{u}$ :

$$
\left\langle\left\langle v^{s}, v^{u}\right\rangle=0 .\right.
$$

We can show, using the uniform hyperbolicity, that $\langle\langle\cdot, \cdot\rangle\rangle$ is equivalent to and as smooth as $\langle\cdot, \cdot\rangle$. That is why it is also called adapted metric. Letting $\|\cdot\| \cdot \|$ denote the norm induced by $\langle\langle\cdot, \cdot\rangle\rangle$, if $v^{s} \in E^{s} \backslash\{0\}$ then

$$
\left\|d f v^{s}\right\|^{2}=2 \sum_{n \geq 0} \lambda^{-2 n}\left\|d f^{n}\left(d f v^{s}\right)\right\|^{2}=\lambda^{2}\left[\left\|v^{s}\right\|^{2}-2\right]<\lambda^{2}\left\|v^{s}\right\|^{2},
$$

hence $\left\|d f v^{s}\right\|<\lambda\left\|v^{s}\right\|$. Similarly, if $v^{u} \in E^{u} \backslash\{0\}$ then $\left\|d f^{-1} v^{u}\right\|<\lambda\left\|v^{u}\right\|$.

When $M$ is a surface, the information of the Lyapunov inner product at each $x \epsilon$ $M$ can be recorded by three parameters $s(x), u(x), \alpha(x)$, which we now introduce. The bundles $E^{s}, E^{u}$ are one-dimensional, so there are vectors $e_{x}^{s} \in E_{x}^{s}$ and $e_{x}^{u} \in E_{x}^{u}$, unitary in the metric $\langle\cdot, \cdot\rangle$. In the Lyapunov inner product $\langle\langle\cdot, \cdot\rangle\rangle$, we have that $\left\|e_{x}^{s}\right\|,\left\|e_{x}^{u}\right\| \in\left[\sqrt{2}, C \lambda \sqrt{\frac{2}{\lambda^{2}-\kappa^{2}}}\right]$ are uniformly bounded away from zero and infinity 2 ,

2. Indeed: $2<\left\|e_{x}^{s}\right\|^{2}=2 \sum_{n \geq 0} \lambda^{-2 n}\left\|d f^{n} e_{x}^{s}\right\|^{2} \leq 2 C^{2} \sum_{n \geq 0}\left(\frac{\kappa}{\lambda}\right)^{2 n}=\frac{2 C^{2} \lambda^{2}}{\lambda^{2}-\kappa^{2}}$. 
PARAmeters $s(x), u(x), \alpha(x)$ :

$$
\begin{aligned}
& s(x)=\left\|e_{x}^{s}\right\|=\sqrt{2}\left(\sum_{n \geq 0} \lambda^{-2 n}\left\|d f^{n} e_{x}^{s}\right\|^{2}\right)^{1 / 2} \\
& u(x)=\left\|e_{x}^{u}\right\|=\sqrt{2}\left(\sum_{n \geq 0} \lambda^{-2 n}\left\|d f^{-n} e_{x}^{u}\right\|^{2}\right)^{1 / 2} \\
& \alpha(x)=\angle\left(E_{x}^{s}, E_{x}^{u}\right) .
\end{aligned}
$$

As observed, $s(x), u(x)$ are uniformly bounded away from zero and infinity. Since the splitting $E^{s} \oplus E^{u}$ is continuous, $\alpha(x)$ is also uniformly bounded away from zero and $\pi$.

1.4. Diagonalization of derivative. For the ease of exposition, we continue assuming that $M$ is a surface. We now use the Lyapunov inner product (or, more specifically, the parameters $s(x), u(x), \alpha(x))$ to represent $d f_{x}$ as a hyperbolic matrix. Let $e_{1}=\left[\begin{array}{l}1 \\ 0\end{array}\right]$ and $e_{2}=\left[\begin{array}{l}0 \\ 1\end{array}\right]$ be the canonical basis of $\mathbb{R}^{2}$.

LiNEAR MAP $C(x):$ For $x \in M$, let $C(x): \mathbb{R}^{2} \rightarrow T_{x} M$ be the linear map s.t.

$$
C(x): e_{1} \mapsto \frac{e_{x}^{s}}{s(x)}, C(x): e_{2} \mapsto \frac{e_{x}^{u}}{u(x)} .
$$

The linear transformation $C(x)$ sends the canonical inner product on $\mathbb{R}^{2}$ to the Lyapunov inner product $\left\langle\langle\cdot \cdot \cdot\rangle\right.$ on $T_{x} M$. For a geometer, this may be a simple description of $\langle\langle\cdot \cdot \cdot\rangle\rangle$, but for practical reasons we do not explore such description. Instead, we study the relation of $C(x)$ with the parameters $s(x), u(x), \alpha(x)$. Given a linear transformation, let $\|\cdot\|$ denote its sup norm and $\|\cdot\|_{\text {Frob }}$ its Frobenius norm ${ }^{3}$ These two norms are equivalent, with $\|\cdot\| \leq\|\cdot\|_{\text {Frob }} \leq \sqrt{2}\|\cdot\|$. The next lemma proves that $C$ diagonalizes $d f$.

Lemma 1.1. There is $\mathscr{L}>1$ s.t. the following holds for all $x \in M$ :

(1) $\|C(x)\|_{\text {Frob }} \leq 1$ and $\left\|C(x)^{-1}\right\|_{\text {Frob }}=\frac{\sqrt{s(x)^{2}+u(x)^{2}}}{|\sin \alpha(x)|}$, with $\|C(x)\|,\left\|C(x)^{-1}\right\| \leq \mathscr{L}$.

(2) $C(f(x))^{-1} \circ d f_{x} \circ C(x)$ is a diagonal matrix with diagonal entries $A, B \in \mathbb{R}$ s.t. $|A|,\left|B^{-1}\right|<\lambda$.

Proof. (1) In the basis $\left\{e_{1}, e_{2}\right\}$ of $\mathbb{R}^{2}$ and the basis $\left\{e_{x}^{s},\left(e_{x}^{s}\right)^{\perp}\right\}$ of $T_{x} M, C(x)$ takes the form $\left[\begin{array}{cc}\frac{1}{s(x)} & \frac{\cos \alpha(x)}{u(x)} \\ 0 & \frac{\sin \alpha(x)}{u(x)}\end{array}\right]$, hence $\|C(x)\|_{\text {Frob }}^{2}=\frac{1}{s(x)^{2}}+\frac{1}{u(x)^{2}} \leq 1$. The inverse of $C(x)$ is $\left[\begin{array}{cc}s(x) & -\frac{s(x) \cos \alpha(x)}{\sin \alpha(x)} \\ 0 & \frac{u(x)}{\sin \alpha(x)}\end{array}\right]$, therefore $\left\|C(x)^{-1}\right\|_{\text {Frob }}=\frac{\sqrt{s(x)^{2}+u(x)^{2}}}{|\sin \alpha(x)|}$. Finally, since $s(x), u(x), \alpha(x)$ are uniformly bounded away from zero and infinity, there is $\mathscr{L}>1$ s.t. $\|C(x)\|,\left\|C(x)^{-1}\right\| \leq \mathscr{L}$ for all $x \in M$.

(2) It is clear that $e_{1}, e_{2}$ are eigenvectors of $C(f(x))^{-1} \circ d f_{x} \circ C(x)$. We start calculating the eigenvalue of $e_{1}$. Since $d f e_{x}^{s}= \pm\left\|d f e_{x}^{s}\right\| e_{f(x)}^{s},\left[d f_{x} \circ C(x)\right]\left(e_{1}\right)=$

${ }^{3}$ The Frobenius norm of a $2 \times 2$ matrix $A=\left[\begin{array}{ll}a & b \\ c & d\end{array}\right]$ is $\|A\|_{\text {Frob }}=\sqrt{a^{2}+b^{2}+c^{2}+d^{2}}$. 
$d f_{x}\left[\frac{e_{x}^{s}}{s(x)}\right]= \pm \frac{\left\|d f e_{x}^{s}\right\|}{s(x)} e_{f(x)}^{s}$, hence $\left[C(f(x))^{-1} \circ d f_{x} \circ C(x)\right]\left(e_{1}\right)= \pm\left\|d f e_{x}^{s}\right\| \frac{s(f(x))}{s(x)} e_{1}$. Thus $A:= \pm\left\|d f e_{x}^{s}\right\| \frac{s(f(x))}{s(x)}$ is the eigenvalue of $e_{1}$. Since

$$
s(f(x))^{2}=\frac{2 \lambda^{2}}{\left\|d f e_{x}^{s}\right\|^{2}} \sum_{n \geq 1} \lambda^{-2 n}\left\|d f^{n} e_{x}^{s}\right\|^{2}=\frac{\lambda^{2}}{\left\|d f e_{x}^{s}\right\|^{2}}\left(s(x)^{2}-2\right)<\frac{\lambda^{2} s(x)^{2}}{\left\|d f e_{x}^{s}\right\|^{2}},
$$

we have $|A|<\lambda$. Similarly, $B:= \pm\left\|d f e_{x}^{u}\right\| \frac{u(f(x))}{u(x)}$ is the eigenvalue of $e_{2}$. Observing that $\left\|d f^{-1} e_{f(x)}^{u}\right\| \cdot\left\|d f e_{x}^{u}\right\|=1$, we have

$$
u(f(x))^{2}=2+\sum_{n \geq 1} \lambda^{-2 n}\left\|d f^{-n} e_{f(x)}^{u}\right\|^{2}=2+\frac{u(x)^{2}}{\lambda^{2}\left\|d f e_{x}^{u}\right\|^{2}}=2+\frac{u(f(x))^{2}}{B^{2} \lambda^{2}}>\frac{u(f(x))^{2}}{B^{2} \lambda^{2}},
$$

and so $|B|>\lambda^{-1}$.

Although $s, u, \alpha, C$ depend on the choice of $\lambda$, we will not emphasize this dependence because all calculations will be made for some a priori fixed $\lambda$.

1.5. Lyapunov charts, change of coordinates. From now on, we assume that $f$ is $C^{1+\beta}$. The next step is to compose the linear transformation $C(x)$ with the exponential map to obtain a local chart of $M$ in which $f$ itself becomes a small perturbation of a hyperbolic matrix. Since this is a natural consequence of the use of the Lyapunov inner product, we will call these charts Lyapunov charts, as in BP13, Section 6.4.2]. Fix a small number $\varepsilon \in\left(0, \mathfrak{r}_{0}\right)$ (how small depends on a finite number of inequalities that $\varepsilon$ has to satisfy). Let $Q=\varepsilon^{3 / \beta}$.

LyAPUNOV CHART: The Lyapunov chart at $x$ is the map $\Psi_{x}:[-Q, Q]^{2} \rightarrow M$ defined by $\Psi_{x}:=\exp _{x} \circ C(x)$.

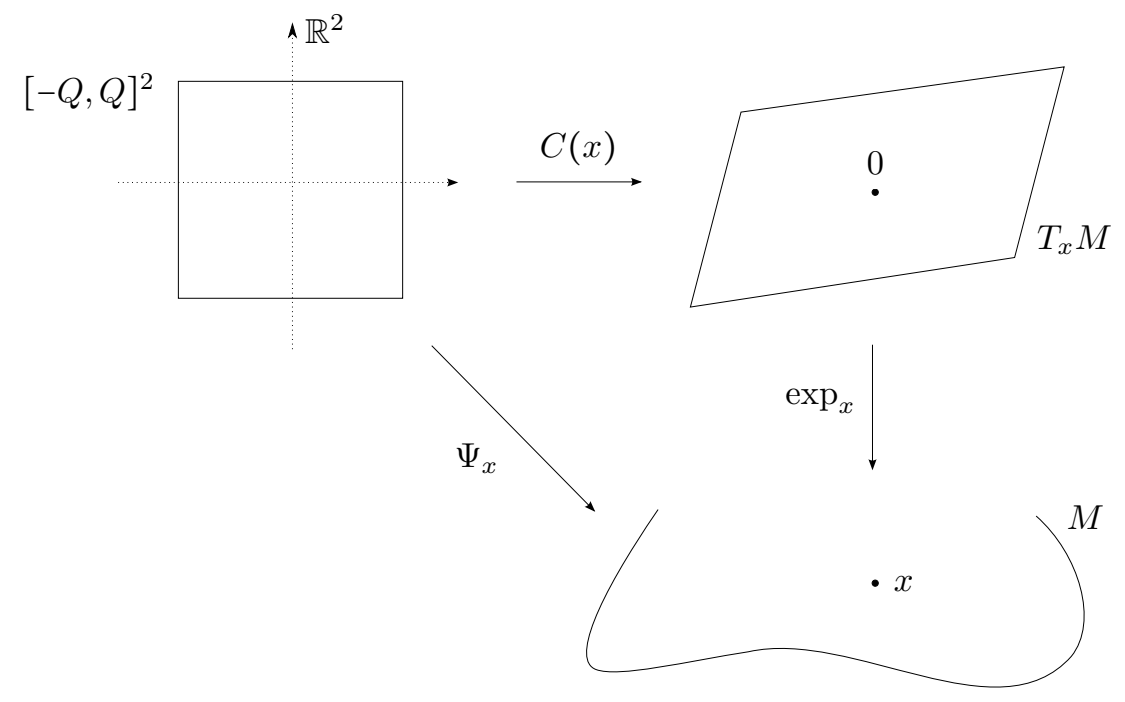

FiguRE 4. The Lyapunov chart $\Psi_{x}$ at $x$.

Since $Q<\varepsilon<\mathfrak{r}_{0}$ and $C(x)$ is a contraction, we have $C(x)\left([-Q, Q]^{2}\right) \subset B_{x}\left[2 \mathfrak{r}_{0}\right]$ and so $\Psi_{x}$ is a diffeomorphism onto its image. By Lemma 1.1 (1), $\Psi_{x}$ is 2-Lipschitz and its inverse is $2 \mathscr{L}$-Lipschitz. In Lyapunov charts, $f$ takes the form $f_{x}:=\Psi_{f(x)}^{-1}$ 。 
$f \circ \Psi_{x}$. The next theorem shows that $f_{x}$ is a small perturbation of a hyperbolic matrix.

Theorem 1.2. The following holds for all $\varepsilon>0$ small enough.

(1) $d\left(f_{x}\right)_{0}=C(f(x))^{-1} \circ d f_{x} \circ C(x)=\left[\begin{array}{cc}A & 0 \\ 0 & B\end{array}\right]$ with $|A|,\left|B^{-1}\right|<\lambda$, cf. Lemma 1.1 .

(2) $f_{x}\left(v_{1}, v_{2}\right)=\left(A v_{1}+h_{1}\left(v_{1}, v_{2}\right), B v_{2}+h_{2}\left(v_{1}, v_{2}\right)\right)$ for $\left(v_{1}, v_{2}\right) \in[-Q, Q]^{2}$ where:

(a) $h_{1}(0,0)=h_{2}(0,0)=0$ and $\nabla h_{1}(0,0)=\nabla h_{2}(0,0)=0$.

(b) $\left\|h_{1}\right\|_{1+\beta / 2}<\varepsilon$ and $\left\|h_{2}\right\|_{1+\beta / 2}<\varepsilon$, where the norms are taken in $[-Q, Q]^{2}$.

A similar statement holds for $f_{x}^{-1}:=\Psi_{x}^{-1} \circ f^{-1} \circ \Psi_{f(x)}$.

Proof. Property (1) is clear since $d\left(\Psi_{x}\right)_{0}=C(x)$ and $d\left(\Psi_{f(x)}\right)_{0}=C(f(x))$. By Lemma $1.1 d\left(f_{x}\right)_{0}=\left[\begin{array}{cc}A & 0 \\ 0 & B\end{array}\right]$ with $|A|,\left|B^{-1}\right|<\lambda$. Define $h_{1}, h_{2}:[-Q, Q]^{2} \rightarrow \mathbb{R}$ by $f_{x}\left(v_{1}, v_{2}\right)=\left(A v_{1}+h_{1}\left(v_{1}, v_{2}\right), B v_{2}+h_{2}\left(v_{1}, v_{2}\right)\right)$. Then (a) is automatically satisfied. It remains to prove (b), which will follow estimating $\left\|d\left(f_{x}\right)_{w_{1}}-d\left(f_{x}\right)_{w_{2}}\right\|$. For the inexperienced reader, we suggest to make the calculation in the euclidean situation (hence all exponential maps are identity). Below we do the general case. For $i=1,2$, define

$$
A_{i}=d\left(\exp _{f(x)}^{-1}{\overline{\left(f \circ \exp _{x}\right.}}_{\left(w_{i}\right)}, B_{i}=d \widetilde{\exp _{x}\left(w_{i}\right)}, C_{i}=d\left(\overline{\left.\exp _{x}\right)_{w_{i}}} .\right.\right.
$$

We first estimate $\left\|A_{1} B_{1} C_{1}-A_{2} B_{2} C_{2}\right\|$. Note that:

- $A_{1}, A_{2}$ are derivatives of the map $\exp _{f(x)}^{-1}$ at nearby points, and so $\left\|A_{1}-A_{2}\right\| \leq$ $\mathscr{H}\left\|w_{1}-w_{2}\right\|$, where $\mathscr{H}>0$ is a constant that only depends on the regularity of exponential maps and their inverses.

- $B_{1}, B_{2}$ are derivatives of $f$ at nearby points, so $\left\|B_{1}-B_{2}\right\| \leq 2 \mathfrak{K}\left\|w_{1}-w_{2}\right\|^{\beta}$.

- $C_{1}, C_{2}$ are derivatives of exponential maps at nearby points, so $\left\|C_{1}-C_{2}\right\| \leq$ $\mathscr{H}\left\|w_{1}-w_{2}\right\|$.

Applying some triangle inequalities, we obtain that

$$
\left\|A_{1} B_{1} C_{1}-A_{2} B_{2} C_{2}\right\| \leq 24 \mathfrak{K} \mathscr{H}\left\|w_{1}-w_{2}\right\|^{\beta} .
$$

Now we estimate $\left\|d\left(f_{x}\right)_{w_{1}}-d\left(f_{x}\right)_{w_{2}}\right\|$ :

$$
\begin{aligned}
& \left\|d\left(f_{x}\right)_{w_{1}}-d\left(f_{x}\right)_{w_{2}}\right\| \leq\left\|C(f(x))^{-1}\right\|\left\|A_{1} B_{1} C_{1}-A_{2} B_{2} C_{2}\right\|\|C(x)\| \\
& \leq 24 \mathfrak{K} \mathscr{H} \mathscr{L}\left\|w_{1}-w_{2}\right\|^{\beta} .
\end{aligned}
$$

Since $\left\|w_{1}-w_{2}\right\|<4 Q$, if $\varepsilon>0$ is small enough then $24 \mathfrak{K} \mathscr{H} \mathscr{L}\left\|w_{1}-w_{2}\right\|^{\beta / 2} \leq$ $96 \mathfrak{K} \mathscr{H} \mathscr{L} \varepsilon^{3 / 2}<\varepsilon$, hence $\left\|d\left(f_{x}\right)_{w_{1}}-d\left(f_{x}\right)_{w_{2}}\right\| \leq \varepsilon\left\|w_{1}-w_{2}\right\|^{\beta / 2}$.

1.6. Graph transforms: construction of invariant manifolds. A consequence of the hyperbolic behavior of $f_{x}$ is that it sends curves that are almost parallel to the vertical axis to curves with the same property; similarly, the inverse map $f_{x}^{-1}$ sends curves that are almost parallel to the horizontal axis to curves with the same property. This geometrical feature allows to construct local stable and unstable manifolds. According to Anosov Ano69, pp. 23], this construction has been more or less known to Darboux, Poincaré and Lyapunov, but their proofs required additional assumptions on the system. Hadamard and Perron were the ones to observe that hyperbolicity is a sufficient condition. Below, we explain the method of Hadamard. The idea is to find the local invariant manifolds among graphs of functions, which we call admissible manifolds. The maps $f_{x}^{ \pm 1}$ define 
operators on the spaces of admissible manifolds, called graph transforms, and the local invariant manifolds are limit points of compositions of such operators. As already mentioned, usually $f$ is only assumed to be $C^{1}$, but we take $f \in C^{1+\beta}$ to keep the analogy with the remaining of the text, and make use of the Lyapunov charts constructed in Subsection 1.5.

AdMissible MANIFOLDS: An $s$-admissible manifold at $\Psi_{x}$ is a set of the form $V^{s}=\Psi_{x}\{(t, F(t)):|t| \leq Q\}$, where $F:[-Q, Q] \rightarrow \mathbb{R}$ is a $C^{1}$ function s.t. $F(0)=$ $0, F^{\prime}(0)=0$ and $\left\|F^{\prime}\right\|_{C^{0}} \approx 0$. Similarly, a $u$-admissible manifold at $\Psi_{x}$ is a set of the form $V^{u}=\Psi_{x}\{(G(t), t):|t| \leq Q\}$, where $G:[-Q, Q] \rightarrow \mathbb{R}$ is a $C^{1}$ function s.t. $G(0)=0, G^{\prime}(0)=0$ and $\left\|G^{\prime}\right\|_{C^{0}} \approx 0$.

We call $F, G$ the representing functions of $V^{s}, V^{u}$ respectively. We prefer not to specify the quantifier for the condition $\left\|F^{\prime}\right\|_{C^{0}},\left\|G^{\prime}\right\|_{C^{0}} \approx 0$. Instead, think of an $s / u$-admissible as an almost horizontal/vertical curve that is tangent to the horizontal/vertical axis at the origin. Let $\mathscr{M}_{x}^{s}, \mathscr{M}_{x}^{u}$ be the space of all $s, u$-admissible manifolds at $\Psi_{x}$ respectively. Introduce a metric on $\mathscr{M}_{x}^{s}$ as follows: for $V_{1}, V_{2} \in \mathscr{M}_{x}^{s}$ with representing functions $F_{1}, F_{2}$, let

$$
\operatorname{dist}\left(F_{1}, F_{2}\right):=\left\|F_{1}-F_{2}\right\|_{C^{0}} .
$$

A similar definition holds for $\mathscr{M}_{x}^{u}$.

GRAPH TRANSFORMS $\mathscr{F}_{x}^{s}, \mathscr{F}_{x}^{u}$ : The stable graph transform $\mathscr{F}_{x}^{s}: \mathscr{M}_{f(x)}^{s} \rightarrow \mathscr{M}_{x}^{s}$ is the map that sends $V^{s} \in \mathscr{M}_{f(x)}^{s}$ to the unique $\mathscr{F}_{x}^{s}\left[V^{s}\right] \in \mathscr{M}_{x}^{s}$ with representing function $F$ s.t. $\Psi_{x}\{(t, F(t)):|t| \leq Q\} \subset f^{-1}\left(V^{s}\right)$. Similarly, the unstable graph transform $\mathscr{F}_{x}^{u}: \mathscr{M}_{x}^{u} \rightarrow \mathscr{M}_{f(x)}^{u}$ is the map that sends $V^{u} \in \mathscr{M}_{x}^{u}$ to the unique $\mathscr{F}_{x}^{u}\left[V^{u}\right] \in \mathscr{M}_{f(x)}^{u}$ with representing function $G$ s.t. $\Psi_{f(x)}\{(G(t), t):|t| \leq Q\} \subset f\left(V^{u}\right)$.

In other words, $\mathscr{F}_{x}^{s}$ sends an $s$-admissible manifold at $\Psi_{f(x)}$ with representing function $F$ to an $s$-admissible manifold at $\Psi_{x}$ whose graph of the representing function is contained in the graph of $f_{x}^{-1} \circ F$, and $\mathscr{F}_{x}^{u}$ sends a $u$-admissible manifold at $\Psi_{x}$ with representing function $G$ to a $u$-admissible manifold at $\Psi_{f(x)}$ whose graph of the representing function is contained in the graph of $f_{x} \circ G$. See Figure 5 below.

Theorem 1.3. $\mathscr{F}_{x}^{s}$ and $\mathscr{F}_{x}^{u}$ are well-defined contractions.

The proof of the theorem follows from the hyperbolicity of $f_{x}$. Using it, we can construct local stable and unstable manifolds.

Stable/unstable MAnifolds: The stable manifold of $x \in M$ is the $s$-admissible manifold $V^{s}[x] \in \mathscr{M}_{x}^{s}$ defined by

$$
V^{s}[x]:=\lim _{n \rightarrow \infty}\left(\mathscr{F}_{x}^{s} \circ \cdots \circ \mathscr{F}_{f^{n-1}(x)}^{s}\right)\left[V_{n}\right]
$$

for some (any) sequence $\left\{V_{n}\right\}_{n \geq 0}$ with $V_{n} \in \mathscr{M}_{f^{n}(x)}^{s}$. The unstable manifold of $x \in M$ is the $u$-admissible manifold $V^{u}[x] \in \mathscr{M}_{x}^{u}$ defined by

$$
V^{u}[x]:=\lim _{n \rightarrow-\infty}\left(\mathscr{F}_{f^{-1}(x)}^{u} \circ \cdots \circ \mathscr{F}_{f^{n}(x)}^{u}\right)\left[V_{n}\right]
$$

for some (any) sequence $\left\{V_{n}\right\}_{n \leq 0}$ with $V_{n} \in \mathscr{M}_{f^{n}(x)}^{u}$.

The sets $V^{s}[x]$ and $V^{u}[x]$ are well-defined because the graph transforms are contractions (Theorem 1.3 above), and they are indeed admissible curves. Note that $V^{s}[x]$ only depends on the future $\left\{f^{n}(x)\right\}_{n \geq 0}$, while $V^{u}[x]$ only depends on 

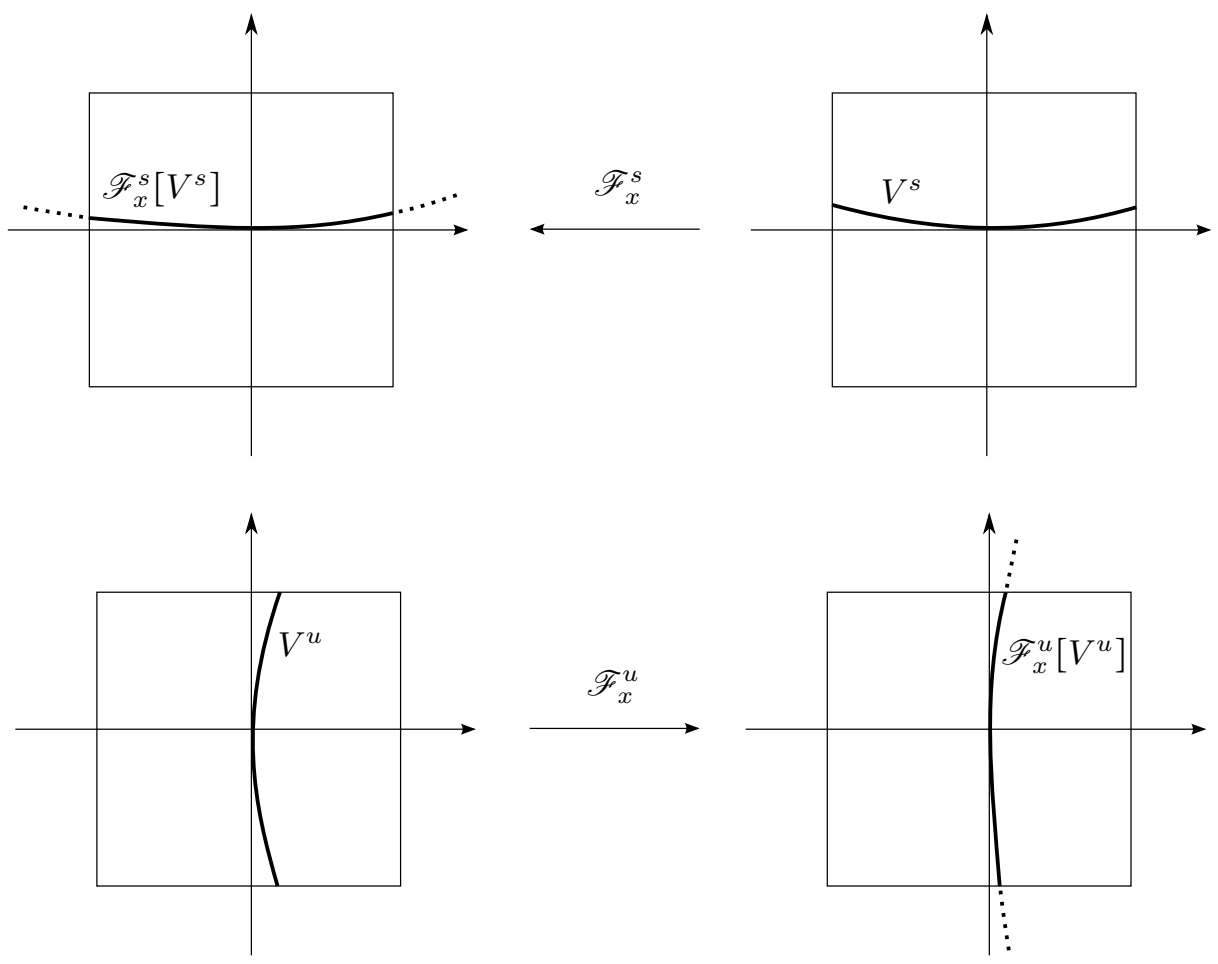

FiguRE 5. Graph transforms: the stable graph transform $\mathscr{F}_{x}^{s}$ sends an $s$-admissible manifold at $\Psi_{f(x)}$ to an $s$-admissible manifold at $\Psi_{x}$, while the unstable graph transform $\mathscr{F}_{x}^{u}$ sends a $u_{-}^{-}$ admissible manifold at $\Psi_{x}$ to a $u$-admissible manifold at $\Psi_{f(x)}$.

the past $\left\{f^{n}(x)\right\}_{n \leq 0}$. Also, since $\Psi_{x}$ and its inverse have uniformly bounded norms (Lemma 1.1(a)), the stable and unstable manifolds have uniform sizes.

1.7. Higher dimensions. We sketch how to make the construction in higher dimension. As we have done, the definition of Lyapunov inner product works in any dimension, but not the definition of $C(x)$. Since this matrix is used to send the canonical inner product of $\mathbb{R}^{2}$ to the Lyapunov inner product on $T_{x} M$, in arbitrary dimension we can similarly define $C(x): \mathbb{R}^{n} \rightarrow T_{x} M$ to be a linear transformation s.t. $\langle v, w\rangle_{\mathbb{R}^{n}}=\left\langle\langle C(x) v, C(x) w\rangle\right.$ for all $v, w \in \mathbb{R}^{n}$, i.e. $C(x)$ is an isometry between $\left(\mathbb{R}^{n},\langle\cdot, \cdot\rangle_{\mathbb{R}^{n}}\right)$ and $\left(T_{x} M,\langle\langle\cdot, \cdot\rangle\rangle\right)$. Let $d_{s}, d_{u} \in \mathbb{N}$ be the dimensions of $E^{s}, E^{u}$. The map $C(x)$ is not uniquely defined, and we can assume that it sends $\mathbb{R}^{d_{s}} \times\{0\}$ to $E_{x}^{s}$ and $\{0\} \times \mathbb{R}^{d_{u}}$ to $E_{x}^{u}$. Doing this for all $x \in M$, we obtain a family $\{C(x)\}_{x \in M}$ of linear transformations. Although the splitting $T M=E^{s} \oplus E^{u}$ is continuous, we cannot always take $x \in M \mapsto C(x)$ continuously, because $E^{s}$ and $E^{u}$ may have nonreal exponents, causing rotations inside them. But for our purpose, what matters is the behavior of the sequence $\left\{C\left(f^{n}(x)\right)\right\}_{n \in \mathbb{Z}}$ for each $x \in M$. For what it is worth, $x \in M \mapsto C(x)$ can be chosen measurably, see e.g. BO18, Footnote at page 48]. 
The composition $C(f(x))^{-1} \circ d f_{x} \circ C(x)$ takes the block form

$$
C(f(x))^{-1} \circ d f_{x} \circ C(x)=\left[\begin{array}{cc}
D_{s} & 0 \\
0 & D_{u}
\end{array}\right]
$$

where $D_{s}$ is a $d_{s} \times d_{s}$ matrix s.t. $\left\|D_{s} v\right\| \leq \lambda\|v\|$ for all $v \in \mathbb{R}^{d_{s}}$, and $D_{u}$ is a $d_{u} \times d_{u}$ matrix s.t. $\left\|D_{u}^{-1} w\right\| \leq \lambda\|w\|$ for all $w \in \mathbb{R}^{d_{u}}$. This is the higher dimensional counterpart of Lemma 1.1 (2). Define the Lyapunov chart $\Psi_{x}$ as in Subsection 1.5. which satisfies a higher dimensional version of Theorem 1.2 with respect to the above block form. Defining an $s$-admissible manifold at $\Psi_{x}$ as a set of the form $V^{s}=\Psi_{x}\left\{(t, F(t)): t \in[-Q, Q]^{d_{s}}\right\}$, where $F:[-Q, Q]^{d_{s}} \rightarrow \mathbb{R}^{d_{u}}$ is a $C^{1}$ function s.t. $F(0)=0, F^{\prime}(0)=0$ and $\left\|F^{\prime}\right\|_{C^{0}} \approx 0$, and similarly $u$-admissible manifolds at $\Psi_{x}$, Theorem 1.3 holds. Hence, every $x \in M$ has local stable and unstable manifolds.

\section{NONUNIFORMLY HYPERBOLIC SYSTEMS}

As discussed in Section 1, uniformly hyperbolic systems had a big impact on the development of dynamical systems and ergodic theory. Unfortunately, uniform hyperbolicity is a condition that is hard to be satisfied. For instance, if a three dimensional manifold admits an Anosov flow then its fundamental group has exponential growth PT72. During the seventies, new notions of hyperbolicity were proposed. These notions substitute the uniform assumption to weaker ones. One of them, called nonuniform hyperbolicity, was introduced by Pesin Pes76, Pes77a, Pes77b. In contrast to uniform hyperbolicity, which requires hyperbolicity to hold every time (for all $n \in \mathbb{Z}$ or $t \in \mathbb{R}$ ) and everywhere (for all $x \in M$ ), the notion of nonuniform hyperbolicity assumes an asymptotic hyperbolicity (on the average) not necessarily in the whole phase space (almost everywhere), i.e. hyperbolicity occurs but in a nonuniform way.

Here is a simplistic way of comparing uniform and nonuniform hyperbolicity. To prepare the dough for bread, a baker needs to contract and stretch repeatedly the dough. An ideal baker would make such operation at every movement, all over the dough. This is uniform hyperbolicity. On the other hand, a real-life baker performs nonuniform hyperbolicity: he does not make the operation at every movement (he might get tired from time to time) and he can forget some tiny parts of the dough. As it turns out, it is the notion of nonuniform hyperbolicity that allows for applications outside of Mathematics.

At the same time that nonuniform hyperbolicity is weak enough to include many new examples and applications, it is strong enough to recover many of the properties of uniformly hyperbolic systems, such as stable manifolds and graph transforms. This is one of the reasons of the success of the theory of nonuniformly hyperbolic systems, known as Pesin theory. Since its beginning, it constitutes an important tool for the understanding of ergodic and statistical properties of smooth dynamical systems. Nowadays, Pesin theory is classical and there are great textbooks on the topic, see e.g. [FHY83 KM95, BP07. For the applications to symbolic dynamics in Part 2, we follow the modern approach that was recently developed by Sarig [Sar13], which has been slightly improved in the past two years or so [LS19 LM18

Now, we make essential use of the $C^{1+\beta}$ regularity. Indeed, the theory is just not true under $C^{1}$ regularity, see e.g. Pugh's example in [BP13, Chapter 15]. 
2.1. Definitions and examples. Let $M$ be a closed (compact without boundary) connected smooth Riemannian manifold, and let $f: M \rightarrow M$ be a $C^{1}$ diffeomorphism. The objects that identify the asymptotic hyperbolicity are the Lyapunov exponents.

LyAPUNOV EXPONENT: For a nonzero vector $v \in T M$, the Lyapunov exponent of $f$ at $v$ is defined by

$$
\chi(v):=\lim _{n \rightarrow+\infty} \frac{1}{n} \log \left\|d f^{n} v\right\|
$$

when the limit exists.

The mere existence of the limit should not be taken for granted, even for uniformly hyperbolic systems. It comes from the Oseledets theorem, which is a measuretheoretic statement that we now explain. Assume that $\mu$ is a probability measure on $M$, invariant under $f$. For simplicity, assume that $\mu$ is ergodic. The Oseledets theorem proves that, for $\mu$-a.e. $x \in M$, the Lyapunov exponents of every nonzero $v \in T_{x} M$ exist Ose68. Furthermore, they exists and are equal for future or past iterations. In our context, we state it as follows.

Theorem 2.1 (Oseledets). Let $(f, \mu)$ be as above. Then there is a $f$-invariant subset $\widetilde{M} \subset M$ with $\mu[\widetilde{M}]=1$, real numbers $\chi_{1}<\chi_{2}<\cdots<\chi_{k}$, and a splitting $T \widetilde{M}=E^{1} \oplus E^{2} \oplus \cdots \oplus E^{k}$ satisfying:

(1) INVARIANCE: $d f\left(E_{x}^{i}\right)=E_{f(x)}^{i}$ for all $x \in \widetilde{M}$ and $i=1, \ldots, k$.

(2) Lyapunov exponents: For all $x \in \widetilde{M}$ and all nonzero $v \in E_{x}^{i}$,

$$
\chi(v)=\lim _{n \rightarrow \pm \infty} \frac{1}{n} \log \left\|d f^{n} v\right\|=\chi_{i} .
$$

When $\mu$ is not ergodic, we can apply a standard argument of ergodic decomposition to conclude that $\chi(v)$ exists $\mu$-a.e., but now its value depends on $x$. Theorem 2.1 above follows from the general version of the Oseledets theorem on cocycles satisfying an integrability condition, see e.g. the recent survey of Filip Fil19. In our setting, the integrability condition is that $\log \left\|d f^{ \pm 1}\right\| \in L^{1}(\mu)$. Since $f$ is a diffeomorphism on a closed manifold, this condition is automatically satisfied.

The notion of nonuniform hyperbolicity also depends on a measure. Let $(f, \mu)$ be as above, where $\mu$ is not necessarily ergodic.

NONUNIFORMLY HYPERBOLIC DIFFEOMORPHISM: The pair $(f, \mu)$ is called nonuniformly hyperbolic if for $\mu$-a.e. $x \in M$ we have $\chi(v) \neq 0$ for all nonzero $v \in T_{x} M$. In this case, $\mu$ is called a hyperbolic measure.

If $f$ is uniformly hyperbolic (see the notation of Subsection 1.1), then $\chi(v) \leq$ $\log \kappa<0$ for nonzero $v \in E^{s}$, and $\chi(v) \geq-\log \kappa>0$ for nonzero $v \in E^{u}$, wherever the Lyapunov exponents exist. Thus a uniformly hyperbolic diffeomorphism is nonuniformly hyperbolic, for any invariant probability measure. Here is another example: if $f$ is a diffeomorphism and $p \in M$ is a hyperbolic periodic point with period $n$, then $\mu=\frac{1}{n} \sum_{k=0}^{n-1} \delta_{f^{k}(p)}$ is a hyperbolic measure. We will usually assume that the Lyapunov exponents are bounded away from zero.

$\chi$-HYPerbolic measure: Given $(f, \mu)$ nonuniformly hyperbolic and $\chi>0, \mu$ is called $\chi$-hyperbolic if for $\mu$-a.e. $x \in M$ we have $|\chi(v)|>\chi$ for all nonzero $v \in T_{x} M$.

In the latter example, $\mu$ is $\chi$-hyperbolic for all $\chi$ smaller than the multiplier of $p$. Now let $\varphi: M \rightarrow M$ be a flow generated by a vector field $X$ of class $C^{1}$, and 
let $\mu$ be a $\varphi$-invariant probability measure on $M$. Since $d \varphi^{t} \circ X=X \circ \varphi^{t}$, we have $\chi\left(X_{x}\right)=0$ for all $x \in M$, hence the assumption of nonzero exponents is required along the remaining directions.

NONUNIFORMLY HYPERBOLIC FLOW: The pair $(\varphi, \mu)$ is called nonuniformly hyperbolic if for $\mu$-a.e. $x \in M$ we have $\chi(v) \neq 0$ for all $v \in T_{x} M$ not proportional to $X_{x}$. When this happens, the measure $\mu$ is called hyperbolic.

Again, it is easy to see that a uniformly hyperbolic flow is nonuniformly hyperbolic for any invariant probability measure, and that the Dirac measure of a hyperbolic closed orbit is hyperbolic. The notion of $\chi$-hyperbolic measure is defined accordingly.

$\chi$-HYPERBolic MEASURE: Given $(\varphi, \mu)$ nonuniformly hyperbolic and $\chi>0, \mu$ is called $\chi$-hyperbolic if for $\mu$-a.e. $x \in M$ we have $|\chi(v)|>\chi$ for all nonzero $v \in T_{x} M$ transverse to $X_{x}$.

Let us mention some classical examples.

1. The slow down of $f_{A}: \mathbb{T}^{2} \rightarrow \mathbb{T}^{2}$, see [Kat79] and BP13, §1.3].

2. Let $f$ be a $C^{1}$ surface diffeomorphism, and let $\mu$ be an ergodic $f$-invariant probability measure. Let $h=h_{\mu}(f)$ be Kolmogorov-Sinaŭ entropy, and assume that $h>0$. Then $(f, \mu)$ is nonuniformly hyperbolic, as consequence of the Ruelle inequality applied to $f$ and to $f^{-1}$ :

- $f$ has a positive Lyapunov exponent $\chi^{+} \geq h>0$.

- We have $h_{\mu}\left(f^{-1}\right)=h$, and the Lyapunov spectrum of $\left(f^{-1}, \mu\right)$ is minus the Lyapunov spectrum of $(f, \mu)$, hence $f$ has a Lyapunov exponent $\chi^{-}$such that $-\chi^{-} \geq h$, i.e. $\chi^{-} \leq-h<0$. If in addition $h>\chi$, then $\mu$ is $\chi$-hyperbolic.

3. Let $N$ be a closed manifold with nonpositive sectional curvature, e.g. the surface in Figure 6 containing a flat cylinder between two regions of negative curvature. The geodesic flow on $N$, which is defined on $M=T_{1} N$, has a natural invariant

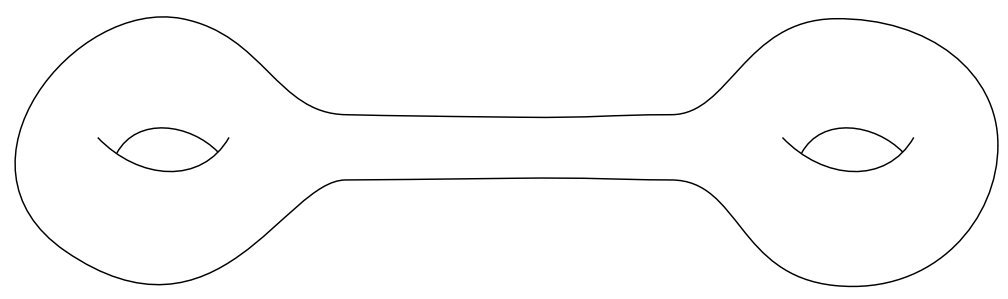

FIgURE 6. Example of a surface with nonpositive curvature.

volume mesure $\mu$. Pesin showed that if the trajectory of a vector $x \in M$ spends a positive fraction of time in regions of negative sectional curvature, then $\chi(v) \neq 0$ for all $v \in T_{x} M$ transverse to $X_{x}$, see [Pes77a. Thm 10.5]. The underlying philosophy (although not entirely correct), is that in regions of negative sectional curvature the derivative behaves like in a uniformly hyperbolic flow, and in regions of zero sectional curvature it only varies linearly, so the overall exponential behavior beats the linear one. Therefore, if $\mu$ is ergodic then it is hyperbolic. Unfortunately, the ergodicity of $\mu$ is still an open problem (even when $N$ is a surface). 
2.2. The nonuniformly hyperbolic locus $\mathrm{NUH}_{\chi}$. As we have done above, the notion of nonuniform hyperbolicity is an almost-everywhere statement that depends on a measure. Due to the Oseledets theorem, we can still get almost-everywhere statements if we only consider Lyapunov regular points, which are points that satisfy Theorem 2.1 and a non-degeneracy assumption on the angles $\angle\left(E^{i}, E^{j}\right)$ between the invariant subbundles. For some applications, this restriction is cumbersome. For example, if $x, y$ are Lyapunov regular, then most likely points in $W^{s}(x) \cap W^{u}(y)$ are not Lyapunov regular (this happens e.g. when $x, y$ have different Lyapunov exponents).

In what follows, we employ a different approach. We fix some $\chi>0$ and consider the set of points satisfying a weaker notion of nonuniform hyperbolicity, that still allows to construct local invariant manifolds. This perspective appeared in an essential way in the work in preparation of the author with Buzzi and Crovisier BCL. Independently and simultaneously, Ben Ovadia recently obtained a similar characterization in higher dimensions BO19.

Let $f: M \rightarrow M$ be a $C^{1}$ diffeomorphism. As in Section 1, we start assuming that $M$ is a closed surface. Let $\chi>0$.

The NONUNIFORMLY HYPERBOlIC LOCUS $\mathrm{NUH}_{\chi}$ : It is the set of points $x \in M$ for which there are transverse unitary vectors $e_{x}^{s}, e_{x}^{u} \in T_{x} M$ s.t.:

(NUH1) $e_{x}^{s}$ contracts in the future at least $-\chi$ and expands in the past:

$$
\limsup _{n \rightarrow+\infty} \frac{1}{n} \log \left\|d f^{n} e_{x}^{s}\right\| \leq-\chi \text { and } \liminf _{n \rightarrow+\infty} \frac{1}{n} \log \left\|d f^{-n} e_{x}^{s}\right\|>0 .
$$

(NUH2) $e_{x}^{u}$ contracts in the past at least $-\chi$ and expands in the future:

$$
\limsup _{n \rightarrow+\infty} \frac{1}{n} \log \left\|d f^{-n} e_{x}^{u}\right\| \leq-\chi \text { and } \liminf _{n \rightarrow+\infty} \frac{1}{n} \log \left\|d f^{n} e_{x}^{u}\right\|>0 .
$$

(NUH3) The parameters $s(x), u(x)$ below are finite:

$$
\begin{aligned}
& s(x)=\sqrt{2}\left(\sum_{n \geq 0} e^{2 n \chi}\left\|d f^{n} e_{x}^{s}\right\|^{2}\right)^{1 / 2} \in[\sqrt{2}, \infty) \\
& u(x)=\sqrt{2}\left(\sum_{n \geq 0} e^{2 n \chi}\left\|d f^{-n} e_{x}^{u}\right\|^{2}\right)^{1 / 2} \in[\sqrt{2}, \infty) .
\end{aligned}
$$

Clearly, $\mathrm{NUH}_{\chi}$ is invariant by $f$. Observe that the definitions of $s(x), u(x)$ are the same as those given in Subsection 1.3. where we change $\lambda$ to $e^{-\chi}$. The first two conditions above guarantee that $e_{x}^{s}, e_{x}^{u}$ are defined up to a sign, and the last condition guarantees asymptotic contractions of rates at least $-\chi$. These conditions are weaker than Lyapunov regularity, hence $\mathrm{NUH}_{\chi}$ contains all Lyapunov regular points with exponents greater than $\chi$ in absolute value. In particular, $\mathrm{NUH}_{\chi}$ carries all $\chi$-hyperbolic measures. But $\mathrm{NUH}_{\chi}$ might contain points with some Lyapunov exponents equal to $\pm \chi$, and even non-regular points, where the contraction rates oscillate infinitely often. Usually, $\mathrm{NUH}_{\chi}$ is a non-compact subset of $M$. Observe that if (NUH3) holds, then the first conditions of (NUH1)-(NUH2) hold as well. In practice, this is how we will show that $x \in \mathrm{NUH}_{\chi}$.

The quality of hyperbolicity can be measured from the parameters $s(x), u(x)$ and from the angle $\alpha(x)=\angle\left(e_{x}^{s}, e_{x}^{u}\right)$. More specifically, $x \in \mathrm{NUH}_{\chi}$ has bad hyperbolicity when at least one of the following situations occur: 
- $s(x)$ is large: it takes a long time to see forward contraction along $e_{x}^{s}$.

- $u(x)$ is large: it takes a long time to see backward contraction along $e_{x}^{u}$.

- $\alpha(x)$ is small: it is hard to distinguish the stable and unstable directions.

None of these situations happen for uniformly hyperbolic systems: as we have seen in Subsection 1.3. for uniformly hyperbolic systems the parameters $s, u, \alpha$ are uniformly bounded away from zero and infinity. For nonuniformly hyperbolic systems, the behaviour is more complicated. Another reason for complication is that, contrary to uniformly hyperbolic systems, the maps $x \in \mathrm{NUH}_{\chi} \mapsto e_{x}^{s}, e_{x}^{u}$ are usually not more than just measurable.

2.3. Diagonalization of derivative. As in Subsection 1.4 we define linear maps $C(x)$ that diagonalize $d f$, the difference being that we only take $x \in \mathrm{NUH}_{\chi}$.

LinEAR MAP $C(x):$ For $x \in \mathrm{NUH}_{\chi}$, let $C(x): \mathbb{R}^{2} \rightarrow T_{x} M$ be the linear map s.t.

$$
C(x): e_{1} \mapsto \frac{e_{x}^{s}}{s(x)}, C(x): e_{2} \mapsto \frac{e_{x}^{u}}{u(x)} .
$$

Above, $\left\{e_{1}, e_{2}\right\}$ is the canonical basis for $\mathbb{R}^{2}$. If, for each $x \in \mathrm{NUH}_{\chi}$, we define a Lyapunov inner product $\langle\langle\cdot \cdot \cdot\rangle\rangle$ on $T_{x} M$, then $C(x)$ sends the canonical metric on $\mathbb{R}^{2}$ to $\left\langle\langle\cdot, \cdot\rangle\right.$. Lemma 1.1 remains valid, except for the uniform bound on $\left\|C(x)^{-1}\right\|$. This result is known as Oseledets-Pesin reduction, see e.g. BP13, Theorem 6.10].

Lemma 2.2 (Oseledets-Pesin reduction). The following holds for all $x \in \mathrm{NUH}_{\chi}$ :

(1) $\|C(x)\|_{\text {Frob }} \leq 1$ and $\left\|C(x)^{-1}\right\|_{\text {Frob }}=\frac{\sqrt{s(x)^{2}+u(x)^{2}}}{|\sin \alpha(x)|}$.

(2) $C(f(x))^{-1} \circ d f_{x} \circ C(x)$ is a diagonal matrix with diagonal entries $A, B \in \mathbb{R}$ s.t. $|A|,\left|B^{-1}\right|<e^{-\chi}$.

The proof is the same as in Lemma 1.1 .

2.4. Pesin charts, the parameter $Q(x)$, change of coordinates. From now on, we assume that $f$ is $C^{1+\beta}$. Remember we are also assuming that $M$ is a closed surface. Fix $\varepsilon \in\left(0, \mathfrak{r}_{0}\right)$ small.

PESIN Chart: The Pesin chart at $x \in \mathrm{NUH}_{\chi}$ is the map $\Psi_{x}:\left[-\varepsilon^{3 / \beta}, \varepsilon^{3 / \beta}\right]^{2} \rightarrow M$ defined by $\Psi_{x}:=\exp _{x} \circ C(x)$.

This is exactly the same definition of Lyapunov chart given in Subsection 1.5 , but we call it Pesin chart for historical reasons. The map $\Psi_{x}$ is well-defined for each $x \in \mathrm{NUH}_{\chi}$. In Pesin charts, $f$ takes the form $f_{x}:=\Psi_{f(x)}^{-1} \circ f \circ \Psi_{x}$. Unfortunately, we might not be able to see hyperbolicity for $f_{x}$, but only for a restriction: while in the uniformly hyperbolic situation $C(x), C(x)^{-1}$ are uniformly bounded, now the parameters $s, u, \alpha$ can degenerate and so $\left\|C(f(x))^{-1}\right\|$ can be arbitrarily large, causing a big distortion. To decrease the domain of definition of $f_{x}$, we multiply its current size by a large negative power of $\left\|C(f(x))^{-1}\right\|$.

Parameter $Q(x):$ For $x \in \mathrm{NUH}_{\chi}$, define $Q(x)=\varepsilon^{3 / \beta}\left\|C(f(x))^{-1}\right\|_{\text {Frob }}^{-12 / \beta}$.

The choice of the powers $3 / \beta$ and $12 / \beta$ is not canonical but just an artifact of the proof, and any choice of powers bigger than these would also make the proof work. This more complicated definition of $Q(x)$ is the price we pay to detect hyperbolicity among nonuniformly hyperbolic systems, as stated in the theorem below. 
Theorem 2.3 (Pesin). The following holds for all $\varepsilon>0$ small. If $x \in \mathrm{NUH}_{\chi}$ then:

(1) $d\left(f_{x}\right)_{0}=C(f(x))^{-1} \circ d f_{x} \circ C(x)=\left[\begin{array}{cc}A & 0 \\ 0 & B\end{array}\right]$ with $|A|,\left|B^{-1}\right|<e^{-\chi}$, cf. Lemma 2.2 .

(2) $f_{x}\left(v_{1}, v_{2}\right)=\left(A v_{1}+h_{1}\left(v_{1}, v_{2}\right), B v_{2}+h_{2}\left(v_{1}, v_{2}\right)\right),\left(v_{1}, v_{2}\right) \in[-10 Q(x), 10 Q(x)]^{2}$, where:

(a) $h_{1}(0,0)=h_{2}(0,0)=0$ and $\nabla h_{1}(0,0)=\nabla h_{2}(0,0)=0$.

(b) $\left\|h_{1}\right\|_{1+\beta / 2}<\varepsilon$ and $\left\|h_{2}\right\|_{1+\beta / 2}<\varepsilon$, with norms taken in $[-10 Q(x), 10 Q(x)]^{2}$.

A similar statement holds for $f_{x}^{-1}=\Psi_{x}^{-1} \circ f^{-1} \circ \Psi_{f(x)}$.

The above statement and proof below are similar to [Sar13. Thm 2.7], see also BP07, Thm 5.6.1].

Proof. We proceed as in the proof of Theorem 1.2. The main difficulty resides on part (2)(b). We still have the estimate

$$
\left\|A_{1} B_{1} C_{1}-A_{2} B_{2} C_{2}\right\| \leq 24 \mathfrak{K} \mathscr{H}\left\|w_{1}-w_{2}\right\|^{\beta},
$$

but now

$$
\begin{aligned}
& \left\|d\left(f_{x}\right)_{w_{1}}-d\left(f_{x}\right)_{w_{2}}\right\| \leq\left\|C(f(x))^{-1}\right\|\left\|A_{1} B_{1} C_{1}-A_{2} B_{2} C_{2}\right\|\|C(x)\| \\
& \leq 24 \mathfrak{K} \mathscr{H}\left\|C(f(x))^{-1}\right\|\left\|w_{1}-w_{2}\right\|^{\beta} .
\end{aligned}
$$

If $w_{1}, w_{2} \in[-10 Q(x), 10 Q(x)]^{2}$ then $\left\|w_{1}-w_{2}\right\|<40 Q(x)$, hence for $\varepsilon>0$ small

$$
24 \mathfrak{K} \mathscr{H}\left\|C(f(x))^{-1}\right\|\left\|w_{1}-w_{2}\right\|^{\beta / 2} \leq 200 \mathfrak{K} \mathscr{H} \varepsilon^{3 / 2}\left\|C(f(x))^{-1}\right\|^{-5} \leq 200 \mathfrak{K} \mathscr{H} \varepsilon^{3 / 2}<\varepsilon .
$$

This completes the proof.

Therefore, at a smaller scale that depends on the quality of hyperbolicity at $x$, the map $f_{x}$ is again the perturbation of a hyperbolic matrix.

2.5. Temperedness and the parameter $q(x)$. After successfully detecting hyperbolicity for $f_{x}$, the next step is to define graph transforms. As seen in Section 1. for uniformly hyperbolic systems the domains of all Lyapunov charts have the same size. Since forward images of $u$-admissible manifolds and backward images of $s$-admissible manifolds grow essentially $\lambda^{-1}$, their images do cross the successive domains from one side to the other, see Figure 5 By Theorem 2.3 , for nonuniformly hyperbolic systems the forward images of $u$-admissible manifolds and backward images of $s$-admissible manifolds grow essentially $e^{\chi}$. Therefore we face a problem when the ratio $\frac{Q(f(x))}{Q(x)}$ is far from 1 :

- If $Q(f(x)) \gg Q(x)$, then the image of a $u$-admissible manifold at $x$ does not cross the domain of $\Psi_{f(x)}$ from top to bottom.

- If $Q(f(x)) \ll Q(x)$, then the image of an $s$-admissible manifold at $f(x)$ does not cross the domain of $\Psi_{x}$ from left to right.

The parameters $s(x), u(x), \alpha(x)$ and $s(f(x)), u(f(x)), \alpha(f(x))$ differ roughly by the action of $d f_{x}$, so there is a constant $\mathscr{C}=\mathscr{C}(f)>1$ s.t. $\mathscr{C}^{-1} \leq \frac{Q(f(x))}{Q(x)} \leq \mathscr{C}$ for all $x \in \mathrm{NUH}_{\chi}$. Nevertheless, this control is yet not enough to rule out the above problems, since we can still have $\mathscr{C} \gg e^{\chi}$. To solve this issue, we need to further reduce the domains of Pesin charts, introducing a parameter that varies regularly. 
Parameter $q(x)$ : For $x \in \mathrm{NUH}_{\chi}$, define $q(x)=\inf \left\{e^{\varepsilon|n|} Q\left(f^{n}(x)\right): n \in \mathbb{Z}\right\}$.

While $Q(x)$ (essentially) does not depend on $\varepsilon$, the parameter $q(x)$ does and, if positive, it does behave nicely along orbits:

$$
e^{-\varepsilon} \leq \frac{q(f(x))}{q(x)} \leq e^{\varepsilon}
$$

The above definition is motivated by the proof of Lemma 1.1.1 in [Pes76], and provides the optimal value for $q(x) \leq Q(x)$ satisfying the above inequalities. This is known as the tempering kernel lemma, see e.g. BP13, Lemma 6.11]. We remark that there are other proofs of the tempering kernel lemma, but that do not provide optimal $q(x)$, see e.g. [BP07, Lemma 3.5.7].

Since $q(x) \leq Q(x)$, the restriction of $f_{x}$ to the smaller domain $[-q(x), q(x)]^{2}$ is a small perturbation of a hyperbolic matrix. Now we are safe: restricting $\Psi_{x}$ to $[-q(x), q(x)]^{2}$, if $\varepsilon>0$ is small enough then the growth of $u / s$-admissible manifolds beats the possible increase/decrease of domains. Motivated by this, we consider the subset of $\mathrm{NUH}_{\chi}$ where $q$ is positive.

ThE NONUNIFORMLY HYPERBOLIC LOCUS NUH*

$$
\mathrm{NUH}_{\chi}^{*}=\left\{x \in \mathrm{NUH}_{\chi}: q(x)>0\right\} .
$$

By the next lemma, $\mathrm{NUH}_{\chi}^{*}$ carries the same finite invariant measures as $\mathrm{NUH}_{\chi}$.

Lemma 2.4. If $\mu$ is an $f$-invariant probability measure supported on $\mathrm{NUH}_{\chi}$, then $\mu$ is supported on $\mathrm{NUH}_{\chi}^{*}$.

Proof. By assumption, $\mu\left[\mathrm{NUH}_{\chi}\right]=1$. Clearly, if $\lim _{n \rightarrow \pm \infty} \frac{1}{n} \log Q\left(f^{n}(x)\right)=0$ then $q(x)>0$. We will prove that $\lim _{n \rightarrow \pm \infty} \frac{1}{n} \log Q\left(f^{n}(x)\right)=0$ for $\mu$-a.e. $x \in \mathrm{NUH}_{\chi}$. Define the function $\varphi: \mathrm{NUH}_{\chi} \rightarrow \mathbb{R}$ by

$$
\varphi(x):=\log \left[\frac{Q(f(x))}{Q(x)}\right]=\log Q(f(x))-\log Q(x) .
$$

Since $\mathscr{C}^{-1} \leq \frac{Q(f(x))}{Q(x)} \leq \mathscr{C}$ for $x \in \mathrm{NUH}_{\chi}$, we have $\varphi \in L^{1}(\mu)$. Let $\varphi_{n}=\log (Q \circ$ $\left.f^{n}\right)-\log Q$ be the $n$-th Birkhoff sum of $\varphi$. By the Birkhoff ergodic theorem, $\lim _{n \rightarrow+\infty} \frac{\varphi_{n}(x)}{n}$ exists $\mu$-a.e. Since by the Poincaré recurrence theorem we have $\liminf _{n \rightarrow+\infty}\left|\varphi_{n}(x)\right|=\liminf _{n \rightarrow+\infty}\left|\log Q\left(f^{n}(x)\right)-\log Q(x)\right|<\infty \mu$-a.e, it follows that $\lim _{n \rightarrow+\infty} \frac{\varphi_{n}(x)}{n}=0$ for $\mu$-a.e. $x \in \mathrm{NUH}_{\chi}$. Proceeding in the same way for $n \rightarrow-\infty$, we conclude that $\lim _{n \rightarrow \pm \infty} \frac{1}{n} \log Q\left(f^{n}(x)\right)=0$ for $\mu$-a.e. $x \in \mathrm{NUH}_{\chi}$.

2.6. Sizes of invariant manifolds: the parameters $q^{s}(x), q^{u}(x)$. Using what we have done so far, we can proceed as in Section 1 to construct invariant manifolds: define $s / u$-admissible manifolds as graphs of functions $F:[-q(x), q(x)] \rightarrow \mathbb{R}$ satisfying some regularity assumptions (that we will explain later), and define graph transforms $\mathscr{F}_{x}^{s}, \mathscr{F}_{x}^{u}$. Hence Theorem 1.3 holds, so we can construct (local) stable and unstable manifolds for every $x \in \mathrm{NUH}_{\chi}^{*}$. This is essentially what is done in Pesin theory, see e.g. BP07, Chapter 7].

In general, $q(x)$ is not the optimal size for the local invariant manifolds, and in some applications we need bigger sizes for them. This is the case for the construction of countable Markov partitions that we will discuss in Part 2. Observe that $q(x)$ might be small for two different reasons: 
- There is $n>0$ for which $e^{\varepsilon n} Q\left(f^{n}(x)\right)$ is small.

- There is $n>0$ for which $e^{\varepsilon n} Q\left(f^{-n}(x)\right)$ is small.

In the first case, the forward behavior of $Q\left(f^{n}(x)\right)$ is bad, so we expect to construct a small stable manifold; but we are also constructing a small unstable manifold, i.e. the bad forward behaviour is influencing the size of the unstable manifold! Since the unstable manifold only depends on the past, its size should not be affected by the future. To deal with this, we introduce two new parameters $q^{s}(x)$ and $q^{u}(x)$, the first controlling the future behavior and the second controlling the past behavior. Then we use them to construct invariant manifolds with larger sizes.

Parameters $q^{s}(x)$ AND $q^{u}(x)$ : For $x \in \mathrm{NUH}_{\chi}^{*}$, define

$$
\begin{aligned}
q^{s}(x) & =\inf \left\{e^{\varepsilon n} Q\left(f^{n}(x)\right): n \geq 0\right\} \\
q^{u}(x) & =\inf \left\{e^{\varepsilon n} Q\left(f^{-n}(x)\right): n \geq 0\right\} .
\end{aligned}
$$

In other words, $q^{s}(x), q^{u}(x)$ are the one-sided versions of $q(x)$. Just like $q$, the parameters $q^{s}, q^{u}$ depend on $\varepsilon$. We will use $q^{s}(x)$ as the scale for considering the stable graph transform and $q^{u}(x)$ as the scale for considering the unstable graph transform.

Lemma 2.5. For all $x \in \mathrm{NUH}_{\chi}^{*}$, the following holds:

(1) Good Definition: $q^{s}(x), q^{u}(x)>0$ and $q(x)=\min \left\{q^{s}(x), q^{u}(x)\right\}$.

(2) Greedy algorithm:

$$
\begin{aligned}
q^{s}(x) & =\min \left\{e^{\varepsilon} q^{s}(f(x)), Q(x)\right\} \\
q^{u}(x) & =\min \left\{e^{\varepsilon} q^{u}\left(f^{-1}(x)\right), Q(x)\right\} .
\end{aligned}
$$

The proofs are direct, see also [LM18, Lemma 4.2].

2.7. Graph transforms: construction of invariant manifolds. There are dynamical explanations for Lemma 2.5(2). Let us discuss the first equality. Assume that $s$-admissible manifolds at $x$ have representing functions defined in the interval $\left[-q^{s}(x), q^{s}(x)\right]$. If $\varepsilon>0$ is small enough, then the stable graph transform $\mathscr{F}_{x}^{s}$ takes the graph of a representing function defined in $\left[-q^{s}(f(x)), q^{s}(f(x))\right]$ and expands it at least by a factor of $e^{\varepsilon}$, so the new representing function is well-defined in $\left[-e^{\varepsilon} q^{s}(f(x)), e^{\varepsilon} q^{s}(f(x))\right]$. Since its domain of definition should not go beyond $[-Q(x), Q(x)]$ (where we have a good control on $f_{x}$ ), the best we can do is to define it in $\left[-q^{s}(x), q^{s}(x)\right]$. In summary, $q^{s}$ provides maximal scales for the definition of stable graph transforms. Similarly, $q^{u}$ provides maximal scales for the definition of unstable graph transforms. With this in mind, we give a new definition of $s / u$-admissible manifolds.

Admissible MANifolds: An $s$-admissible manifold at $\Psi_{x}$ is a set of the form $V^{s}=\Psi_{x}\left\{(t, F(t)):|t| \leq q^{s}(x)\right\}$, where $F:\left[-q^{s}(x), q^{s}(x)\right] \rightarrow \mathbb{R}$ is a $C^{1+\beta / 3}$ function s.t. $F(0)=F^{\prime}(0)=0$ and $\left\|F^{\prime}\right\|_{0}+\operatorname{Hol}_{\beta / 3}\left(F^{\prime}\right) \leq \frac{1}{2}$, where the norms are taken in $\left[-q^{s}(x), q^{s}(x)\right]$. Similarly, a $u$-admissible manifold at $\Psi_{x}$ is a set of the form $V^{u}=$ $\Psi_{x}\left\{(G(t), t):|t| \leq q^{u}(x)\right\}$, where $G:\left[-q^{u}(x), q^{u}(x)\right] \rightarrow \mathbb{R}$ is a $C^{1+\beta / 3}$ function s.t. $G(0)=G^{\prime}(0)=0$ and $\left\|G^{\prime}\right\|_{0}+\operatorname{Hol}_{\beta / 3}\left(G^{\prime}\right) \leq \frac{1}{2}$, with norms taken in $\left[-q^{u}(x), q^{u}(x)\right]$.

As before, $F, G$ are called the representing functions of $V^{s}, V^{u}$ respectively, and let $\mathscr{M}_{x}^{s}, \mathscr{M}_{x}^{u}$ be the space of all $s, u$-admissible manifolds at $\Psi_{x}$ respectively, which are metric spaces with the $C^{0}$ distance. Let $x \in \mathrm{NUH}_{\chi}^{*}$. 
GRAPH TRANSFORMS $\mathscr{F}_{x}^{s}, \mathscr{F}_{x}^{u}$ : The stable graph transform $\mathscr{F}_{x}^{s}: \mathscr{M}_{f(x)}^{s} \rightarrow \mathscr{M}_{x}^{s}$ is the map that sends $V^{s} \in \mathscr{M}_{f(x)}^{s}$ to the unique $\mathscr{F}_{x}^{s}\left[V^{s}\right] \in \mathscr{M}_{x}^{s}$ with representing function $F$ s.t. $\Psi_{x}\left\{(t, F(t)):|t| \leq q^{s}(x)\right\} \subset f^{-1}\left(V^{s}\right)$. Similarly, the unstable graph transform $\mathscr{F}_{x}^{u}: \mathscr{M}_{x}^{u} \rightarrow \mathscr{M}_{f(x)}^{u}$ is the map that sends $V^{u} \in \mathscr{M}_{x}^{u}$ to the unique $\mathscr{F}_{x}^{u}\left[V^{u}\right] \in \mathscr{M}_{f(x)}^{u}$ with representing function $G$ s.t. $\Psi_{f(x)}\left\{(G(t), t):|t| \leq q^{u}(f(x))\right\} \subset f\left(V^{u}\right)$.

The difference from the previous definition is that the stable and unstable graph transforms are defined at different scales, see Figure 7 below.
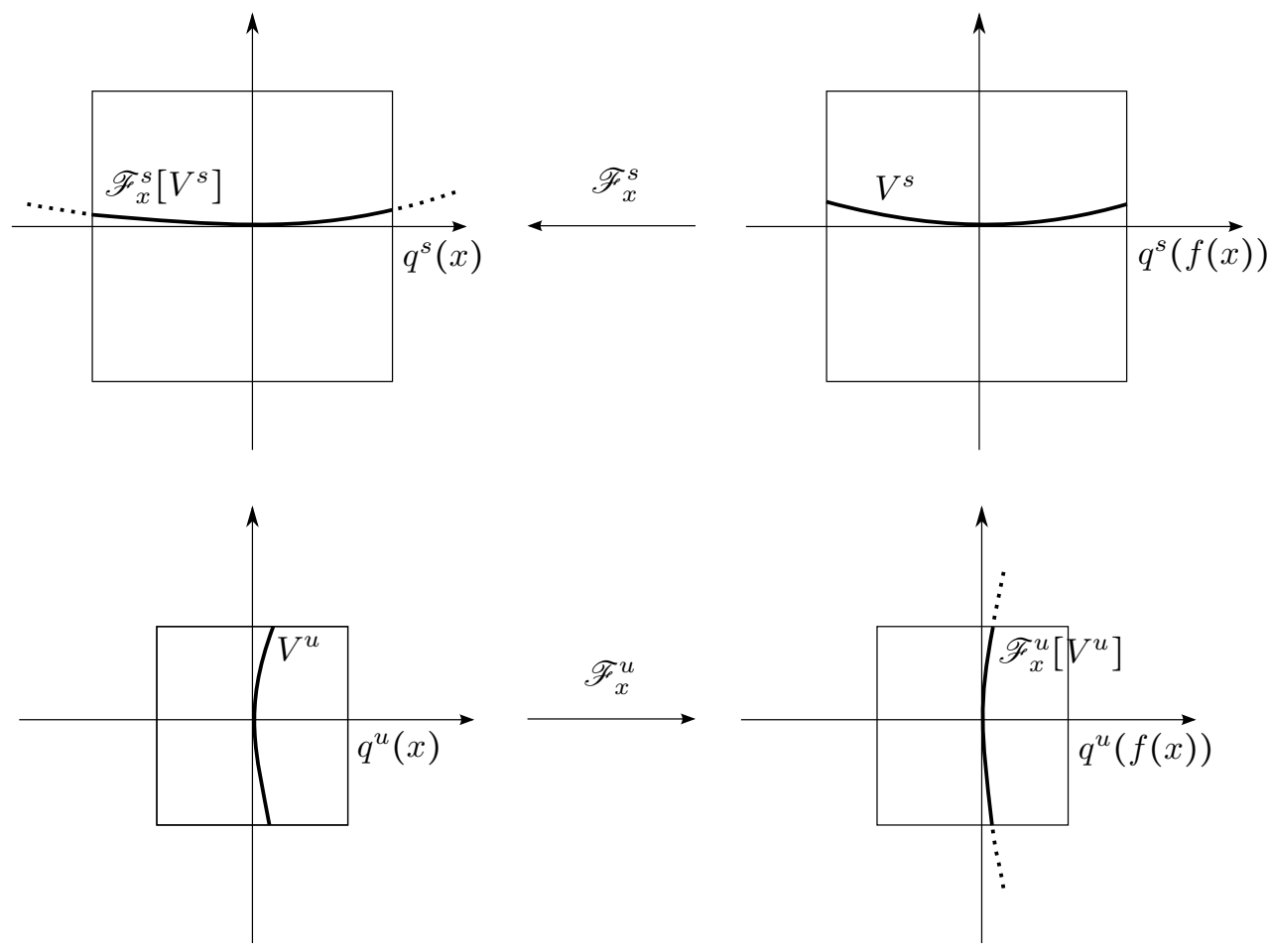

FiguRE 7. The stable graph transforms are defined at scales $q^{s}$, and the unstable graph transforms at scales $q^{u}$.

Theorem 2.6. $\mathscr{F}_{x}^{s}$ and $\mathscr{F}_{x}^{u}$ are well-defined contractions.

For nonuniformly hyperbolic systems, this theorem was first proved by Pesin, see Pes76, Thm. 2.2.1]. The proof is similar to the proof of Theorem 1.3. In its present form, with scales $q^{s}$ and $q^{u}$, the above result is a special case of [Sar13, Prop. 4.12]. For $x \in \mathrm{NUH}_{\chi}^{*}$, let $V^{s}[x]$ and $V^{u}[x]$ be the stable and unstable manifolds of $x$, defined as in Subsection 1.6. Then $V^{s}[x]$ is the image under $\Psi_{x}$ of the graph of a function defined in $\left[-q^{s}(x), q^{s}(x)\right]$, while $V^{u}[x]$ is the image under $\Psi_{x}$ of the graph of a function defined in $\left[-q^{u}(x), q^{u}(x)\right]$.

2.8. Higher dimensions. Now consider diffeomorphisms in any dimension. The discussion follows [BO18 and in some sense [BO19]. We can no longer perform the construction using only the parameters $s(x), u(x), \alpha(x)$, because now the spaces 
$E^{s}, E^{u}$ are higher dimensional, and each vector defines its own parameter. More specifically, consider the following definition, for each fixed $\chi>0$.

The NONUNiformly hyPERBolic LOCUS $\mathrm{NUH}_{\chi}$ : It is the set of points $x \in M$ for which there is a splitting $T_{x} M=E_{x}^{s} \oplus E_{x}^{u}$ s.t.:

(NUH1) Every $v \in E_{x}^{s}$ contracts in the future at least $-\chi$ and expands in the past:

$$
\limsup _{n \rightarrow+\infty} \frac{1}{n} \log \left\|d f^{n} v\right\| \leq-\chi \text { and } \liminf _{n \rightarrow+\infty} \frac{1}{n} \log \left\|d f^{-n} v\right\|>0 .
$$

(NUH2) Every $v \in E_{x}^{u}$ contracts in the past at least $-\chi$ and expands in the future:

$$
\limsup _{n \rightarrow+\infty} \frac{1}{n} \log \left\|d f^{-n} v\right\| \leq-\chi \text { and } \liminf _{n \rightarrow+\infty} \frac{1}{n} \log \left\|d f^{n} v\right\|>0 .
$$

(NUH3) The parameters $s(x)=\sup _{\substack{v \in E^{s} s \\\|v\|=1}} S(x, v)$ and $u(x)=\sup _{\substack{w \in E_{x}^{u} \\\|w\|=1}} U(x, w)$ are finite, where:

$$
\begin{aligned}
S(x, v) & =\sqrt{2}\left(\sum_{n \geq 0} e^{2 n \chi}\left\|d f^{n} v\right\|^{2}\right)^{1 / 2}, \\
U(x, w) & =\sqrt{2}\left(\sum_{n \geq 0} e^{2 n \chi}\left\|d f^{-n} w\right\|^{2}\right)^{1 / 2} .
\end{aligned}
$$

In BO19, this definition is similar to the definition of the set $\chi$-summ. Again, $\mathrm{NUH}_{\chi}$ is $f$-invariant, and for each $x \in \mathrm{NUH}_{\chi}$ we can define a linear transformation $C(x): \mathbb{R}^{n} \rightarrow T_{x} M$ that sends the canonical metric on $\mathbb{R}^{n}$ to the Lyapunov inner product $\langle\langle\cdot \cdot \cdot\rangle\rangle$ on $T_{x} M$. We again have the block representation

$$
C(f(x))^{-1} \circ d f_{x} \circ C(x)=\left[\begin{array}{cc}
D_{s} & 0 \\
0 & D_{u}
\end{array}\right],
$$

where $D_{s}$ is a $d_{s} \times d_{s}$ matrix s.t. $\left\|D_{s} v\right\| \leq e^{-\chi}\|v\|$ for all $v \in \mathbb{R}^{d_{s}}$ and $D_{u}$ is a $d_{u} \times d_{u}$ matrix s.t. $\left\|D_{u}^{-1} w\right\| \leq e^{-\chi}\|w\|$ for all $w \in \mathbb{R}^{d_{u}}$. This is the higher dimensional OseledetsPesin reduction, see Lemma 2.2(2). Define the Pesin chart $\Psi_{x}$ as in Subsection 2.4 . and the parameter $Q(x)=\mathscr{H}\left\|C(f(x))^{-1}\right\|^{-48 / \beta}$, where $\mathscr{H}=\mathscr{H}(\beta, \varepsilon)$ is a constant that allows to keep the estimates of order $\varepsilon$ and to absorb multiplicative constants. Then a higher dimensional version of Theorem 2.3 holds, see [BO18, Thm 1.13]. From now on, we can repeat the two-dimensional construction, defining the parameters $q(x), q^{s}(x), q^{u}(x)$, the nonuniformly hyperbolic locus $\mathrm{NUH}_{\chi}^{*}$, an $s$-admissible manifold at $\Psi_{x}$ as a set of the form $V^{s}=\Psi_{x}\left\{(t, F(t)): t \in\left[-q^{s}(x), q^{s}(x)\right]^{d_{s}}\right\}$, where $F:\left[-q^{s}(x), q^{s}(x)\right]^{d_{s}} \rightarrow \mathbb{R}^{d_{u}}$ is a $C^{1+\beta / 3}$ function s.t. $F(0)=F^{\prime}(0)=0$ and $\left\|F^{\prime}\right\|_{0}+\operatorname{Hol}_{\beta / 3}\left(F^{\prime}\right) \leq \frac{1}{2}$, where the norms are taken in $\left[-q^{s}(x), q^{s}(x)\right]^{d_{s}}$, and similarly $u$-admissible manifolds. Then Theorem 1.3 holds, see BO18, Prop. 2.8], and so every $x \in \mathrm{NUH}_{\chi}^{*}$ has local stable and unstable manifolds.

\section{MAPS With Discontinuities AND BOUNDED DERIVATIVE}

In the previous section, we considered diffeomorphisms defined on closed (compact without boundary) surfaces. There are natural examples that do not fit into this context, for example Poincaré return maps of flows and billiard maps. Their common feature is the presence of discontinuities, and the possible explosion of derivatives. In the next two sections, we will discuss how to adapt the methods of Section 2 to cover these examples, focusing on the changes that are needed to make 
the arguments work. We start dealing with surface maps with discontinuities and bounded derivative. The reference is LS19.

3.1. Definitions and examples. Let $M$ be a compact surface, possibly with boundary. To avoid multiplicative constants in the calculations, we assume that $M$ has diameter smaller than one. Let $\mathscr{D}^{+}, \mathscr{D}^{-}$be closed subsets of $M$, and consider a map $f: M \backslash \mathscr{D}^{+} \rightarrow M$ with inverse $f^{-1}: M \backslash \mathscr{D}^{-} \rightarrow M$. Let $\mathscr{D}:=\mathscr{D}^{+} \cup \mathscr{D}^{-}$be the set of discontinuities of $f$. We require $f, f^{-1}$ to be local $C^{1+\beta}$ diffeomorphisms.

REgUlarity of $f$ : There is a constant $\mathscr{L}>0$ with the following property:

- For every $x \in M \backslash \mathscr{D}^{+}$there is an open set $U \ni x$ s.t. $f \uparrow_{U}$ is a diffeomorphism onto its image with $C^{1+\beta}$ norm at most $\mathscr{L}$.

○ For every $x \in M \backslash \mathscr{D}^{-}$there is an open set $V \ni x$ s.t. $f^{-1} \uparrow_{V}$ is a diffeomorphism onto its image with $C^{1+\beta}$ norm at most $\mathscr{L}$.

In particular, $\left\|d f^{ \pm 1}\right\|$ is bounded away from zero and infinity, so the integrability condition in the Oseledets theorem holds for any $f$-invariant probability measure. The main difficulty when dealing with $f$ as above is that, as $x$ approaches $\mathscr{D}$, the open sets $U, V$ become smaller, hence the domains of Pesin charts also need to be smaller. To avoid this issue, we only consider trajectories that do not approach $\mathscr{D}$ exponentially fast.

Here is the example to have in mind. Let $N$ be a three dimensional closed Riemannian manifold, let $X$ be a $C^{1+\beta}$ vector field on $N$ s.t. $X(p) \neq 0$ for all $p \in N$, and let $\varphi=\left\{\varphi^{t}\right\}_{t \in \mathbb{R}}$ be the flow generated by $X$. We can reduce the dynamics of $\varphi$ to the dynamics of a surface map by constructing a global Poincaré section $M$ for $\varphi$ as follows:

- Fix $\varepsilon>0$ small enough.

- For each $p \in N$, consider a closed differentiable disc $D(p)$ centered at $p$ with diameter smaller than $\varepsilon$ s.t. $\angle\left(T_{q} D(p), X(q)\right)>\frac{\pi}{2}-\varepsilon$ for all $q \in D(p)$.

- Let $\operatorname{FB}(p):=\bigcup_{|t| \leq \varepsilon} \varphi^{t}[D(p)]$ be the flow box defined by $D(p)$. Using that $X \neq 0$, we see that $\mathrm{FB}(p)$ contains an open ball centered at $p$.

- By compactness, $N$ is covered by finitely many flow boxes $\mathrm{FB}\left(p_{1}\right), \ldots, \mathrm{FB}\left(p_{\ell}\right)$.

Therefore $M=D\left(p_{1}\right) \cup \cdots \cup D\left(p_{\ell}\right)$ is a global Poincaré section for $\varphi$. With some extra work, we can make the discs $D\left(p_{1}\right), \ldots, D\left(p_{\ell}\right)$ to be pairwise disjoint, hence the return time function $\mathfrak{t}: M \rightarrow(0, \infty)$ is bounded away from zero and infinity. See [LS19, Section 2] for details.

Let $f: M \rightarrow M$ be the Poincaré return map of $M$, i.e. $f(x)=\varphi^{\mathrm{t}(x)}(x)$. The map $f$ has discontinuities, with $\left.\mathscr{D}^{ \pm}=\left\{x \in M: f^{ \pm 1}(x) \in \partial M\right\}\right\}^{4}$ See Figure 8 , Nevertheless, where $f^{ \pm 1}$ is continuous, its $C^{1+\beta}$ norm is uniformly bounded. This occurs because, at continuity points, $f^{ \pm 1}$ has the form $\varphi^{\tau}$ where $\tau$ has uniformly bounded $C^{1+\beta}$ norm, see LS19, Lemma 2.5] for details.

3.2. Nonuniform hyperbolicity. To apply the methods of Section 2, we only consider trajectories that do not approach $\mathscr{D}$ exponentially fast. Let $d$ be the distance in $M$.

The NONUNifORMLY HYPERBolic LOCUS $\mathrm{NUH}_{\chi}^{*}$ : It is the set of points $x \in M$ satisfying conditions (NUH1)-(NUH3) of page 19 and the additional condition:

\footnotetext{
${ }^{4}$ Observe that in this case $f$ is defined on all of $M$, but $f^{ \pm 1}$ is discontinuous on $\mathscr{D}^{ \pm}$.
} 


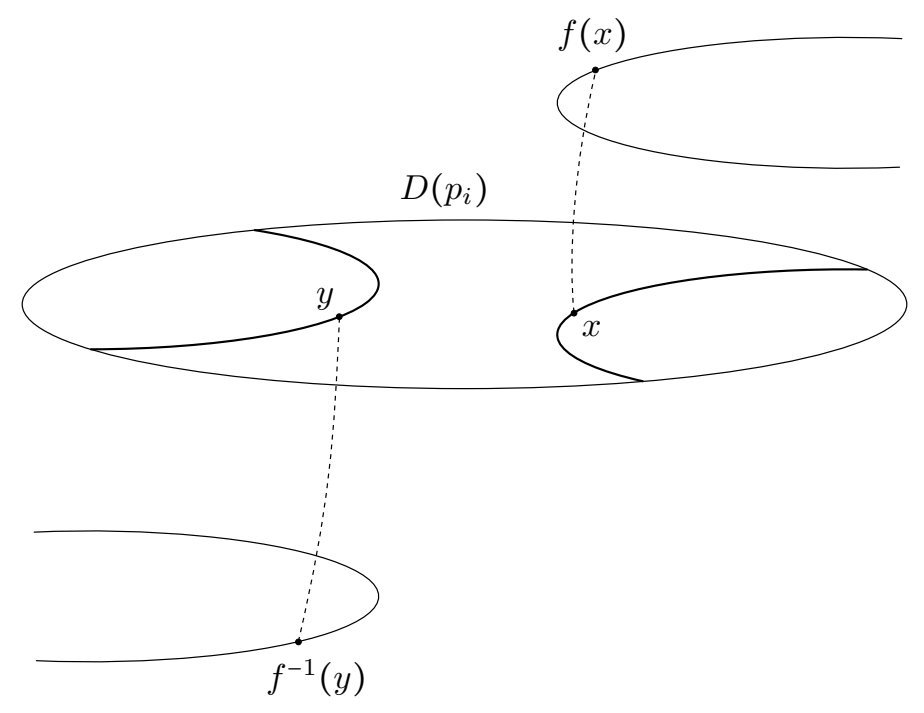

Figure 8. Discontinuities for $f$ : in the picture, $x \in \mathscr{D}^{+}$and $y \in \mathscr{D}^{-}$.

(NUH4) Subexponential CONVERGence to $\mathscr{D}$ :

$$
\lim _{n \rightarrow \pm \infty} \frac{1}{n} \log d\left(f^{n}(x), \mathscr{D}\right)=0 .
$$

The idea of looking at trajectories satisfying condition (NUH4) is not new. It goes back to Sinal in the context of billiards Sin70, which we will discuss in Section 4. See also the section "Overcoming influence of singularities" in KSLP86. At the level of invariant measures, (NUH4) is related to the following notion.

$f$-ADAPTED MEASURE: An $f$-invariant measure on $M$ is called $f$-adapted if the function $\log d(x, \mathscr{D}) \in L^{1}(\mu)$. A fortiori $\mu(\mathscr{D})=0$.

By the Birkhoff ergodic theorem, if $\mu$ is $f$-adapted then (NUH4) holds $\mu$-a.e. If in addition $\mu$ is $\chi$-hyperbolic, then (NUH1)-(NUH3) hold $\mu$-a.e. and therefore $\mu$ is carried by $\mathrm{NUH}_{\chi}$, i.e. $\mu\left[\mathrm{NUH}_{\chi}\right]=1$.

For each $x \in \mathrm{NUH}_{\chi}$, the linear map $C(x)$ can be defined as before, and Lemma 2.2 remains valid with the same proof. To define the Pesin chart $\Psi_{x}$, we just need to adjust its domain of definition, according to the distance of $x$ to $\mathscr{D}$. Let $\delta(x)=\varepsilon^{3 / \beta} d(x, \mathscr{D})$.

Pesin Chart: The Pesin chart at $x \in \mathrm{NUH}_{\chi}$ is the map $\Psi_{x}:[-\delta(x), \delta(x)]^{2} \rightarrow M$ defined by $\Psi_{x}:=\exp _{x} \circ C(x)$.

We also redefine $Q(x)$ accordingly. Let $\rho(x):=d\left(\left\{f^{-1}(x), x, f(x)\right\}, \mathscr{D}\right)$.

PARAmeter $Q(x)$ : For $x \in \mathrm{NUH}_{\chi}$, let $Q(x)=\varepsilon^{3 / \beta} \min \left\{\left\|C(f(x))^{-1}\right\|_{\text {Frob }}^{-12 / \beta}, \varepsilon \rho(x)\right\}$. 
With this definition ${ }^{5}$, the representation of $f$ in Pesin charts $f_{x}:=\Psi_{f(x)}^{-1} \circ f \circ \Psi_{x}$ is well-defined in $[-10 Q(x), 10 Q(x)]^{2}$. Indeed, $Q(x) \leq \varepsilon \delta(f(x))$ and so

$$
\left(f \circ \Psi_{x}\right)\left([-10 Q(x), 10 Q(x)]^{2}\right) \subset \Psi_{f(x)}\left([-\delta(f(x)), \delta(f(x))]^{2}\right) .
$$

Again, in the domain $[-10 Q(x), 10 Q(x)]^{2}$ the map $f_{x}$ is a small perturbation of a hyperbolic matrix.

Now define the parameters $q, q^{s}, q^{u}$, the set $\mathrm{NUH}_{\chi}^{*}$, and the graph transforms $\mathscr{F}_{x}^{s / u}$ as in the previous section, then construct local invariant manifolds for each $x \in \mathrm{NUH}_{\chi}^{*}$. We finish this section proving an analogue of Lemma 2.4 .

Lemma 3.1. If $\mu$ is an $f$-invariant probability measure supported on $\mathrm{NUH}_{\chi}$, then $\mu$ is supported on $\mathrm{NUH}_{\chi}^{*}$.

Proof. By assumption, $\lim _{n \rightarrow \pm \infty} \frac{1}{n} \log d\left(f^{n}(x), \mathscr{D}\right)=0$ for $\mu$-a.e. $x \in M$. Let $\widetilde{Q}(x)=\varepsilon^{3 / \beta}\left\|C(f(x))^{-1}\right\|_{\text {Frob }}^{-12 / \beta}$ be the "old" $Q$. Since $d f$ is uniformly bounded, we can proceed exactly as in Lemma 2.4 to conclude that $\lim _{n \rightarrow \pm \infty} \frac{1}{n} \log \widetilde{Q}\left(f^{n}(x)\right)=0$ for $\mu$-a.e. $x \in M$. But then $\lim _{n \rightarrow \pm \infty} \frac{1}{n} \log Q\left(f^{n}(x)\right)=0$ for $\mu$-a.e. $x \in M$.

\section{Maps With Discontinuities AND UnBounded DERIVATIVE}

Next, we consider surface maps with discontinuities and unbounded derivative. Added to the difficulty that Pesin charts are defined in smaller domains, now $\left\|d f^{ \pm 1}\right\|$ can approach zero and infinity, so even the integrability condition in the Oseledets theorem is no longer automatic. The first development of Pesin theory in this context was KSLP86, whose interest was to apply it for billiard maps. Since its beginning, ergodic theory of billiard maps was mainly focused in a reference Liouville measure. This is the case in KSLP86, where the authors construct invariant manifolds Lebesgue almost everywhere and use them to prove ergodic theoretic properties such as the Ruelle inequality. Contrary to this, in the sequel we follow the same approach of Section 2 not focusing on a particular measure but rather on the set of points with some hyperbolicity. The reference for this section is [LM18.

4.1. Definitions and examples. Let $M$ be a compact surface, possibly with boundary. Again, we assume that $M$ has diameter smaller than one. Let $\mathscr{D}^{+}, \mathscr{D}^{-}$be closed subsets of $M$, and consider $f: M \backslash \mathscr{D}^{+} \rightarrow M$ with inverse $f^{-1}: M \backslash \mathscr{D}^{-} \rightarrow M$. Let $\mathscr{D}:=\mathscr{D}^{+} \cup \mathscr{D}^{-}$be the set of discontinuities of $f$. We require the following conditions on $f$.

REgUlarity of $f$ : There are constants $0<\beta<1<a$ and $\mathfrak{K}>0$ s.t. for all $x \in M \backslash \mathscr{D}$ there is $d(x, \mathscr{D})^{a}<\mathfrak{r}(x)<d(x, \mathscr{D})$ s.t. if $D_{x}=B(x, \mathfrak{r}(x))$ then the following holds:

○ If $y \in D_{x}$ then $\left\|d f_{y}^{ \pm 1}\right\| \leq d(x, \mathscr{D})^{-a}$.

○ If $y_{1}, y_{2} \in D_{x}$ and $f\left(y_{1}\right), f\left(y_{2}\right) \in D_{x^{\prime}}$ then $\left\|\widetilde{d f_{y_{1}}}-\widetilde{d f_{y_{2}}}\right\| \leq \mathfrak{K} d\left(y_{1}, y_{2}\right)^{\beta}$, and if $y_{1}, y_{2} \in D_{x}$ and $f^{-1}\left(y_{1}\right), f^{-1}\left(y_{2}\right) \in D_{x^{\prime \prime}}$ then $\left\|\widetilde{d f_{y_{1}}^{-1}}-\widetilde{d f_{y_{2}}^{-1}}\right\| \leq \mathfrak{K} d\left(y_{1}, y_{2}\right)^{\beta}$.

The first assumption says that $d f^{ \pm 1}$ blows up at most polynomially fast, and the second says that $d f^{ \pm 1}$ is locally $\beta$-Hölder. The examples to have in mind are billiard maps, as we now explain. Given a compact domain $T \subset \mathbb{R}^{2}$ or $T \subset \mathbb{T}^{2}$ with

\footnotetext{
${ }^{5}$ We take the chance to observe that the definition of $Q(x)$ in LS19 has a small error, since it does not depend on $d(f(x), \mathscr{D})$ and so we cannot guarantee that $f_{x}$ is well-defined, see LS19. Thm 3.2 and Corollary 3.6]. Nevertheless, this can be easily fixed with the definition we give here.
} 
piecewise $C^{3}$ boundary, consider the straight line motion of a particle inside $T$, with specular reflections in $\partial T$. The phase space of configurations is $M=\partial T \times\left[-\frac{\pi}{2}, \frac{\pi}{2}\right]$ with the convention that $(r, \theta) \in M$ represents $r=$ collision position at $\partial T$ and $\theta=$ angle of collision. Given $(r, \theta) \in M$, let $\left(r^{+}, \theta^{+}\right)$be the next collision and $\left(r^{-}, \theta^{-}\right)$ be the previous collision. Let $\left\{r_{1}, \ldots, r_{k}\right\}$ be the break points of $\partial T$, and define:

$$
\begin{aligned}
& \mathscr{D}^{+}=\left\{r^{+}=r_{i} \text { for some } i\right\} \cup\left\{\theta^{+}= \pm \frac{\pi}{2}\right\} \\
& \mathscr{D}^{-}=\left\{r^{-}=r_{i} \text { for some } i\right\} \cup\left\{\theta^{-}= \pm \frac{\pi}{2}\right\} .
\end{aligned}
$$

The billiard map is $f: M \backslash \mathscr{D}^{+} \rightarrow M$ defined by $f(r, \theta)=\left(r^{+}, \theta^{+}\right)$, with inverse $f: M \backslash \mathscr{D}^{-} \rightarrow M$ defined by $f(r, \theta)=\left(r^{-}, \theta^{-}\right)$. Since $\partial T$ (usually) has two normal vectors at $r_{i}$, we cannot define $f^{ \pm 1}(r, \theta)$ if $r^{ \pm}=r_{i}$. When $\theta^{ \pm}= \pm \frac{\pi}{2}$, the trajectory has a grazing collision, and $f^{ \pm 1}$ is usually discontinuous on $(r, \theta)$. Furthermore, $d f^{ \pm 1}$ becomes arbitrarily large in a neighborhood of $(r, \theta)$. This justifies the choice of $\mathscr{D}^{ \pm}$above. See CM06 for details.

Sinal showed that $f$ has a natural invariant Liouville measure $\mu_{\mathrm{SRB}}=\cos \theta d r d \theta$, which is ergodic for dispersing billiards [Sin70]. Bunimovich constructed examples of ergodic nowhere dispersing billiards Bun74a, Bun74b, Bun79]. These billiards, known as Bunimovich billiards, are nonuniformly hyperbolic. See some examples in Figure 9. Recently, Baladi and Demers constructed measures of maximal entropy

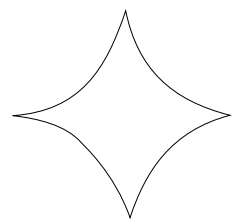

(1)

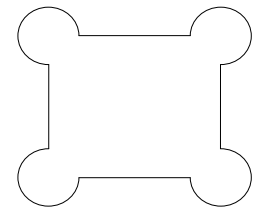

(2)

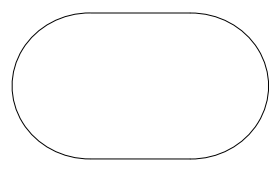

$(3)$

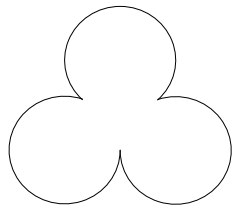

$(4)$

Figure 9. (1) is a Sinaí billiard table. The others are Bunimovich billiard tables: (2) is the pool table with pockets, (3) is the stadium, (4) is the flower.

for some finite horizon periodic Lorentz gases BD20], see more on Section 7 .

4.2. Nonuniform hyperbolicity. We continue only considering trajectories that do not approach $\mathscr{D}$ exponentially fast, and define the nonuniformly hyperbolic locus $\mathrm{NUH}_{\chi}$ as in Subsection 3.2. Similarly, an $f$-invariant measure $\mu$ is called $f$-adapted if $\log d(x, \mathscr{D}) \in L^{1}(\mu)$.

Since $\log \left\|d f^{ \pm 1}\right\|$ is usually unbounded, some measures might not satisfy the integrability condition in the Oseledets theorem. Due to the regularity of $f$, the functions $\log \left\|d f^{ \pm 1}\right\|$ and $\log d(x, \mathscr{D})$ are comparable, therefore $\log \left\|d f^{ \pm 1}\right\| \in L^{1}(\mu)$ iff $\log d(x, \mathscr{D}) \in L^{1}(\mu)$. Hence the Oseledets theorem holds for $f$-adapted measure, which shows that $f$-adaptability is a natural assumption. In particular, if $\mu$ is $f-$ adapted and $\chi$-hyperbolic, then (NUH1)-(NUH4) hold $\mu$-a.e. and so $\mu$ is carried by $\mathrm{NUH}_{\chi}$. For each $x \in \mathrm{NUH}_{\chi}$, we define $C(x)$ as before, and Lemma 2.2 remains valid with the same proof.

Pesin Chart: The Pesin chart at $x \in \mathrm{NUH}_{\chi}$ is $\Psi_{x}:\left[-d(x, \mathscr{D})^{a}, d(x, \mathscr{D})^{a}\right]^{2} \rightarrow M$, defined by $\Psi_{x}:=\exp _{x} \circ C(x)$. 
The definition of $Q(x)$ is more complicated. Let $\rho(x)=d\left(\left\{f^{-1}(x), x, f(x)\right\}, \mathscr{D}\right)$.

Parameter $Q(x)$ : For $x \in \mathrm{NUH}_{\chi}$, define

$$
Q(x)=\varepsilon^{3 / \beta} \min \left\{\left\|C(x)^{-1}\right\|_{\text {Frob }}^{-24 / \beta},\left\|C(f(x))^{-1}\right\|_{\text {Frob }}^{-12 / \beta} \rho(x)^{72 a / \beta}\right\} .
$$

As before, the choice of the powers is not canonical but just an artifact of the proof. The above definition depends on $f^{-1}(x), x, f(x)$, and is strong enough to construct local invariant manifolds, and to run the methods of Part 2. Firstly, in the domain $[-10 Q(x), 10 Q(x)]^{2}$ the representation of $f$ in Pesin charts $f_{x}:=$ $\Psi_{f(x)}^{-1} \circ f \circ \Psi_{x}$ is a small perturbation of a hyperbolic matrix, see LM18, Thm 3.3]. Defining the parameters $q, q^{s}, q^{u}$, the set $\mathrm{NUH}_{\chi}^{*}$, and the graph transforms $\mathscr{F}_{x}^{s / u}$ as before, we construct local invariant manifolds for each $x \in \mathrm{NUH}_{\chi}^{*}$. Finally, we establish an analogue of Lemmas 2.4 and 3.1 .

Lemma 4.1. If $\mu$ is an $f$-adapted probability measure supported on $\mathrm{NUH}_{\chi}$, then $\mu$ is supported on $\mathrm{NUH}_{\chi}^{*}$.

Proof. It is enough to show that

$$
\lim _{n \rightarrow \pm \infty} \frac{1}{n} \log \left\|C\left(f^{n}(x)\right)^{-1}\right\|_{\text {Frob }}=\lim _{n \rightarrow \pm \infty} \frac{1}{n} \log \rho\left(f^{n}(x)\right)=0
$$

for $\mu$-a.e. $x \in M$. By (NUH4), the second equality holds. For the first equality, let $\widetilde{Q}(x)=\varepsilon^{3 / \beta}\left\|C(f(x))^{-1}\right\|_{\text {Frob }}^{-12 / \beta}$ be the "old" $Q$, and observe that the regularity assumption on $f$ implies that $\log \left[\frac{\widetilde{Q} \circ f}{\widetilde{Q}}\right] \in L^{1}(\mu)$ iff $\log d(x, \mathscr{D}) \in L^{1}(\mu)$. Since $\mu$ is $f$-adapted, we get that $\log \left[\frac{\widetilde{Q} \circ f}{\widetilde{Q}}\right] \in L^{1}(\mu)$. Now proceed as in Lemma 2.4 to conclude that $\lim _{n \rightarrow \pm \infty} \frac{1}{n} \log \left\|C\left(f^{n}(x)\right)^{-1}\right\|_{\text {Frob }}=0$ for $\mu$-a.e. $x \in M$.

\section{Part 2. Symbolic dynamics}

Symbolic dynamics is an important tool for the understanding of ergodic and statistical properties of dynamical systems, both smooth and non-smooth. The field of symbolic dynamics is enormous and covers various contexts, from the study of symbolic spaces to the theory of complexity functions, see e.g. Kit98, Fer99. Here, we only discuss the use of symbolic dynamics to represent smooth dynamical systems. The main idea is simple, and can be summarised in two steps: firstly, divide the phase space of a system into finitely or countably many pieces, which we call rectangles; secondly, instead of describing the trajectory of a point by the exact positions in the phase space, just record the sequence of rectangles that the trajectory visits. We call the second step above a coding. This procedure can be made in a wide setting. For instance, any partition defines a coding in the usual way. Such flexibility allows its use in various contexts:

- Periodic points of continuous intervals maps: proof of the Sharkovsky theorem using Markov graphs, see e.g. BH11.

- Milnor-Thurston's kneading theory of continuous intervals maps: description of the trajectory of the critical point with respect to monotonicity intervals MT88.

- Geodesics on surfaces of constant negative curvature: Hadamard represented closed geodesics using sequences of symbols $\operatorname{Had} 98$, see also KU07. 
In this survey, we focus on symbolic dynamics for smooth systems with some hyperbolicity, uniform and nonuniform. The final goal is to describe the invariant measures and ergodic theoretical properties of such systems, and for that a mere coding is not enough: it is important to recover codings, i.e. to know which trajectories are coded in the same way. This reverse procedure is called decoding. Good codings are those for which we can satisfactorily decode. It has long been observed that uniform expansion provides a good decoding: two different trajectories eventually stay far apart and therefore cannot visit the same rectangles. This property, that different trajectories eventually stay far apart, is known as expansivity. It also occurs for (U)-systems, due to the exponential dichotomy of solutions mentioned in Section 1.

Another required property on the coding is that the space of sequences coding the trajectories should be as simple as possible and at the same time rich enough to reflect the structure of the original smooth system. This property is certainly satisfied if the rectangles have the Markov property, since in this case every path on the graph is naturally associated to a genuine orbit. Apart from some technical assumptions, when this occurs we call the partition a Markov partition.

Let us give a simple example of its usefulness. Let $f: K \rightarrow K$ be the horseshoe map described in Example 2 of Subsection 1.1 (Smale's horseshoe). Since $K$ has a fractal structure, it seems rather complicated to understand its periodic points and invariant measures. But the system has a Markov partition that induces a continuous bijection $\pi: \Sigma \rightarrow K$ between the symbolic space $\Sigma=\{0,1\}^{\mathbb{Z}}$ and $K$, that commutes the shift map on $\Sigma$ and the map $f$. Hence we can analyse the dynamical properties of $f$ by means of the dynamical properties of the shift map.

In the next sections, we explain how to construct Markov partitions for smooth systems with some hyperbolicity, both uniform and nonuniform. The conclusion is the existence of a symbolic model. Let us give the definitions. Let $\mathscr{G}=(V, E)$ be an oriented graph. We assume that $V$ is finite or countable, and that for each $v, w \in V$ there is at most one edge $v \rightarrow w$.

TOPOLOGICAL MARKov SHIFT (TMS): Let

$$
\Sigma=\left\{\left\{v_{n}\right\}_{n \in \mathbb{Z}} \in V^{\mathbb{Z}}: v_{n} \rightarrow v_{n+1}, \forall n \in \mathbb{Z}\right\}
$$

be the set of $\mathbb{Z}$-indexed paths on $\mathscr{G}$, and let $\sigma: \Sigma \rightarrow \Sigma$ be the left shift. The pair $(\Sigma, \sigma)$ is called a topological Markov shift.

For short, we will write TMS. An element of $\Sigma$ is denoted by $\underline{v}=\left\{v_{n}\right\}_{n \in \mathbb{Z}}$. We endow $\Sigma$ with the distance $d(\underline{v}, \underline{w}):=\exp \left[-\min \left\{|n|: n \in \mathbb{Z}\right.\right.$ s.t. $\left.\left.v_{n} \neq w_{n}\right\}\right]$. Let $\Sigma^{\#}$ be the recurrent set of $\Sigma$, defined by

$$
\Sigma^{\#}=\left\{\left\{v_{n}\right\}_{n \in \mathbb{Z}} \in \Sigma: \begin{array}{l}
\exists v, w \in V \text { s.t. } v_{n}=v \text { for infinitely many } n>0 \\
\text { and } v_{n}=w \text { for infinitely many } n<0
\end{array}\right\} .
$$

When $V$ is finite, $\Sigma^{\#}=\Sigma$. Let $f: M \rightarrow M$ be a diffeomorphism.

Symbolic MOdel FOR DIFFEOMORPHISM: A symbolic model for $f: M \rightarrow M$ is a triple $(\Sigma, \sigma, \pi)$ where $(\Sigma, \sigma)$ is a TMS and $\pi: \Sigma \rightarrow M$ is a Hölder continuous map

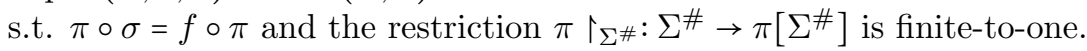

Hence a symbolic model is a TMS together with a projection map $\pi$ that commutes $f$ and $\sigma$, and that is finite-to-one on $\pi\left[\Sigma^{\#}\right]$. A diffeomorphism can have 
many symbolic models. Some of them are bad, when $\pi\left[\Sigma^{\#}\right]$ is much smaller than the subsets where $f$ displays an interesting dynamics. A good symbolic model is one for which $\pi\left[\Sigma^{\#}\right]$ contains the subset where $f$ displays chaotic dynamics. For us, this occurs when $\pi\left[\Sigma^{\#}\right]$ carries $\chi$-hyperbolic measures. To define a symbolic model for flows, we add the flow direction to the TMS.

Topological MARKov Flow (TMF): Given a TMS $(\Sigma, \sigma)$ and a Hölder continuous function $r: \Sigma \rightarrow \mathbb{R}$ with $0<\inf r \leq \sup r<\infty$, define the topological Markov flow $\left(\Sigma_{r}, \sigma_{r}\right)$ by:

○ $\Sigma_{r}=\{(\underline{v}, t): \underline{v} \in \Sigma, 0 \leq t \leq r(\underline{v})\}$ with the identification $(\underline{v}, r(\underline{v})) \sim(\sigma(\underline{v}), 0)$.

○ $\sigma_{r}=\left\{\sigma_{r}^{t}\right\}_{t \in \mathbb{R}}: \Sigma_{r} \rightarrow \Sigma_{r}$ the unit speed vertical flow on $\Sigma_{r}$, called the suspension flow, see Figure 10 .

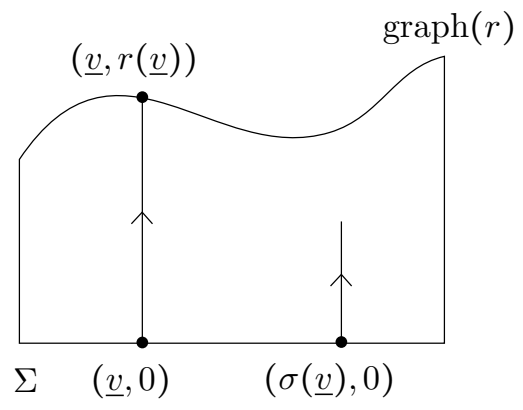

FiguRE 10. The suspension flow $\sigma_{r}$ : starting at $(\underline{v}, 0)$, flow at unit speed until hitting the graph of $r$, then return to the basis via the identification $(\underline{v}, r(\underline{v})) \sim(\sigma(\underline{v}), 0)$ and continue flowing.

An element of $\Sigma_{r}$ is denoted by $(\underline{v}, t)$. Let $\Sigma_{r}^{\#}$ be the recurrent set of $\Sigma_{r}$,

$$
\Sigma_{r}^{\#}=\left\{(\underline{v}, t) \in \Sigma_{r}: \underline{v} \in \Sigma^{\#}\right\}
$$

See LS19 for basic properties on $\left(\Sigma_{r}, \sigma_{r}\right)$. Let $\varphi: M \rightarrow M$ be a flow.

SyMBOLIC MODEL FOR FLOW: A symbolic model for $\varphi: M \rightarrow M$ is a triple $\left(\Sigma_{r}, \sigma_{r}, \pi_{r}\right)$ where $\left(\Sigma_{r}, \sigma_{r}\right)$ is a TMF and $\pi_{r}: \Sigma_{r} \rightarrow M$ is a Hölder continuous map s.t. $\pi_{r} \circ \sigma_{r}^{t}=\varphi^{t} \circ \pi_{r}$ for all $t \in \mathbb{R}$ for which the restriction $\pi_{r} \uparrow_{\Sigma_{r}^{\#}}: \Sigma_{r}^{\#} \rightarrow \pi_{r}\left[\Sigma_{r}^{\#}\right]$ is finite-to-one.

\section{SymbOliC DYNAMICS FOR UNIFORMLY HYPERBOLIC SYSTEMS}

There are at least two general ways of constructing Markov partitions for uniformly hyperbolic systems. One of them, due to Sină, is called the method of successive approximations Sin68b, Sin68a]. The second, due to Bowen, is called the method of pseudo-orbits [Bow08]. Their common feature is the use of the local invariant manifolds constructed in Part 1 as dynamically defined systems of coordinates. For completeness and ease of understanding, we also describe the construction of Adler and Weiss for hyperbolic toral automorphisms [AW67]. Since for nonuniformly hyperbolic systems we will make use of the method of pseudo-orbits, we will only sketch the other techniques, and the details can be found in the original papers. We start defining Markov partitions (and their flow counterpart) in the context of uniformly hyperbolic systems, and explain how they generate symbolic 
models. It is important mentioning that, for uniformly hyperbolic systems, the vertex set $V$ of the oriented graph $\mathscr{G}$ is finite.

5.1. Markov partitions/sections. Let $f: M \rightarrow M$ be a diffeomorphism. As already mentioned, our goal is to construct a partition of $M$ so that the dynamics of $f$ can be represented by a TMS. Let $\mathscr{G}=(V, E)$ be the graph defining the TMS $(\Sigma, \sigma)$. The vertex set $V$ is the set of partition elements, and each edge in $E$ will represent one possible transition by the iteration of $f$. If $v_{0} \rightarrow v_{1}$ and $v_{1} \rightarrow v_{2}$ are edges, then their concatenation is a path from $v_{0}$ to $v_{2}$. This property, translated to the dynamics of $f$, is the Markov property. More precisely, if $R_{0}, R_{1}, R_{2}$ are the partition elements associated to $v_{0}, v_{1}, v_{2}$, then there is a point $x \in R_{0}$ s.t. $f(x) \in R_{1}$ and there is a point $y \in R_{1}$ s.t. $f(y) \in R_{2}$. The Markov property ensures that there is a point $z \in R_{0}$ s.t. $f(z) \in R_{1}$ and $f^{2}(z) \in R_{2}$.

Imagine, for a moment, that $f$ is uniformly expanding. If we define edges $R_{0} \rightarrow R_{1}$ when $f\left(R_{0}\right) \cap R_{1} \neq \varnothing$, then the above property is not guaranteed. If instead we define $R_{0} \rightarrow R_{1}$ when $f\left(R_{0}\right) \supset R_{1}$, then the referred concatenation holds. When $f$ is a uniformly hyperbolic diffeomorphism, the definition of edges requires two inclusions, one for each invariant direction. Let us give the definitions. The references for the discussion in this section are Bow08 for diffeomorphisms and Bow73 for flows. Let $f: M \rightarrow M$ be an Axiom A diffeomorphism, and fix $\varepsilon>0$ small. By Part 1. each $x \in \Omega(f)$ has a local stable manifold $W_{\text {loc }}^{s}(x)=V^{s}[x]$ and a local unstable manifold $W_{\text {loc }}^{u}(x)=V^{u}[x]$. By definition, $W_{\text {loc }}^{s / u}(x)$ is tangent to $E_{x}^{s / u}$ at $x$, hence $W_{\mathrm{loc}}^{s}(x), W_{\mathrm{loc}}^{u}(x)$ are transversal at $x$. The maps $x \in \Omega(f) \mapsto W_{\mathrm{loc}}^{s / u}(x)$ are continuous, see e.g. [Shu87, Thm 6.2(2)]. Hence, if $x, y \in \Omega(f)$ with $\operatorname{dist}(x, y) \ll 1$ then $W_{\text {loc }}^{s}(x)$ and $W_{\text {loc }}^{\bar{u}}(y)$ intersect transversally at a single point. Fix $\delta \ll \varepsilon$, and consider the following definition.

Smale bracket: For $x, y \in \Omega(f)$ with $d(x, y)<\delta$, the Smale bracket of $x$ and $y$ is defined by $\{[x, y]\}:=W_{\mathrm{loc}}^{s}(x) \cap W_{\mathrm{loc}}^{u}(y)$.

For $R \subset \Omega(f)$, let $R^{*}$ denote the interior of $R$ in the induced topology of $\Omega(f)$.

Rectangle: A subset $R \subset \Omega(f)$ is called a rectangle if it satisfies:

(1) Regularity: $R=\overline{R^{\star}}$ and $\operatorname{diam}(R)<\delta$.

(2) Product structure: $x, y \in R \Rightarrow[x, y] \in R$.

The product structure means that $R$ is a rectangle in the system of coordinates given by the local invariant manifolds. Let $W^{s / u}(x, R):=W_{\text {loc }}^{s / u}(x) \cap R$. Regardless $W_{\text {loc }}^{s / u}(x)$ are smooth manifolds, since $\Omega(f)$ is usually a fractal set, then $W^{s / u}(x, R)$ are also usually fractal. It is easy to construct rectangles ${ }^{6}$ Let $\mathscr{R}$ be a finite cover of $\Omega(f)$ by rectangles.

MARKOV PARTITION: $\mathscr{R}$ is called a Markov partition for $f$ if it satisfies:

(1) Disjointness: The elements of $\mathscr{R}$ can only intersect at their boundaries $]^{7}$

(2) Markov property: If $x \in R^{*}$ and $f(x) \in S^{*}$, then

$$
f\left(W^{s}(x, R)\right) \subset W^{s}(f(x), S) \text { and } f^{-1}\left(W^{u}(f(x), S)\right) \subset W^{u}(x, R) .
$$

\footnotetext{
${ }^{6}$ Given $\rho>0$, let $W_{\rho}^{s / u}(x)=W_{\text {loc }}^{s / u}(x) \cap B(x, \rho)$. If $x \in \Omega(f)$, then $\left[W_{\rho}^{u}(x) \cap \Omega(f), W_{\rho}^{s}(x) \cap \Omega(f)\right]$ is a rectangle for all $\rho>0$ small enough.

${ }^{7}$ Boundaries are considered with respect to the relative topology of $W^{s / u}(x, R)$, see Bow08.
} 
If $\mathscr{R}$ only satisfies (2), we call it a Markov cover. The two latter inclusions represent two Markov properties, one for each invariant direction. Geometrically, they assure that if two rectangles intersect, then the intersection occurs all the way from one side to the other, with respect to the system of coordinates of the local invariant manifolds, see Figure 11. We stress that, while here every rectangle has non-empty interior, in the nonuniformly hyperbolic situation we will not be able to guarantee this.

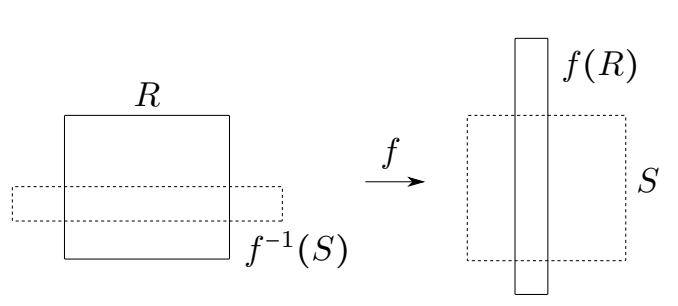

(-) Allowed intersection $\odot$

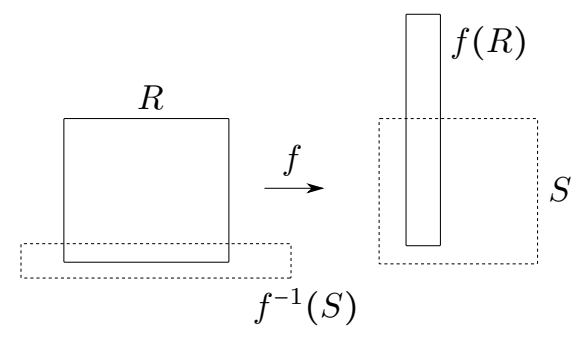

(:) Forbidden interserction $(;)$

Figure 11. The Markov property: if $f(R)$ intersects $S$ nontrivially, then $f(R)$ crosses $S$ completely all the way from one side to the other.

Now let $\varphi: M \rightarrow M$ be an Axiom A flow. Recall the definitions of Subsection 3.1. Given an interval $I \subset \mathbb{R}$ and $Y \subset M$, let $\varphi^{I}(Y):=\bigcup_{t \in I} \varphi^{t}(Y)$.

Proper Section: A finite family $\mathscr{M}=\left\{B_{1}, \ldots, B_{n}\right\}$ is a proper section of size $\alpha$ if there are closed differentiable discs $D_{1}, \ldots, D_{n}$ transverse to the flow direction s.t.:

(1) Closedness: Each $B_{i}$ is a closed subset of $\Omega(\varphi)$.

(2) Cover: $\Omega(\varphi)=\bigcup_{i=1}^{n} \varphi^{[0, \alpha]}\left(B_{i}\right)$.

(3) Regularity: $B_{i} \subset \operatorname{int}\left(D_{i}\right)$ and $\overline{B_{i}^{*}}=B_{i}$, where $B_{i}^{*}$ is the interior of $B_{i}$ in the induced topology of $D_{i} \cap \Omega(\varphi)$.

(4) Partial order: For $i \neq j$, at least one of the sets $D_{i} \cap \varphi^{[0,4 \alpha]}\left(D_{j}\right)$ and $D_{j} \cap \varphi^{[0,4 \alpha]}\left(D_{i}\right)$ is empty; in particular $D_{i} \cap D_{j}=\varnothing$.

For simplicity, denote $B_{1} \cup \cdots \cup B_{n}$ also by $\mathscr{M}$. Let $f: \mathscr{M} \rightarrow \mathscr{M}$ be the Poincaré return map of $\mathscr{M}$, and $\mathfrak{t}: \mathscr{M} \rightarrow(0, \infty)$ the return time function. By properties (2) and (4), $0<\inf \mathfrak{t} \leq \sup \mathfrak{t} \leq \alpha$. By transversality, the stable/unstable directions of $\varphi$ project to stable/unstable directions of the Poincaré map $f$. Also, local invariant manifolds of $f$ are projections, in the flow direction, of local invariant manifolds of $\varphi$, and we can similarly define the Smale bracket $[\cdot, \cdot]$ for $f$.

The maps $f, \mathfrak{t}$ are not continuous, but they are continuous on the subset

$$
\mathscr{M}^{\prime}:=\left\{x \in \mathscr{M}: f^{k}(x) \in \bigcup B_{i}^{*}, \forall k \in \mathbb{Z}\right\} .
$$

Considering points in $\mathscr{M}^{\prime}$ avoids many problems, the first being the definition of the Markov property. We do not want to consider a transition from $B_{i}$ to $B_{j}$ when $f\left(B_{i}\right) \cap B_{j}$ is a subset of $\partial B_{j}$.

Transitions: We write $B_{i} \rightarrow B_{j}$ if there exists $x \in \mathscr{M}^{\prime}$ s.t. $x \in B_{i}, f(x) \in B_{j}$. When this happens, define $\mathscr{T}^{s}\left(B_{i}, B_{j}\right):=\overline{\left\{x \in \mathscr{M}^{\prime}: x \in B_{i}, f(x) \in B_{j}\right\}}$ and $\mathscr{T}^{u}\left(B_{i}, B_{j}\right):=$ $\overline{\left\{y \in \mathscr{M}^{\prime}: y \in B_{j}, f^{-1}(y) \in B_{i}\right\}}$. 
Markov Section: $\mathscr{M}$ is called a Markov section of size $\alpha$ for $\varphi$ if it is a proper section of size $\alpha$ with the following additional properties:

(5) Product Structure: Each $B_{i}$ is a rectangle.

(6) Markov property: If $B_{i} \rightarrow B_{j}$, then

$$
\begin{aligned}
& x \in \mathscr{T}^{s}\left(B_{i}, B_{j}\right) \Rightarrow W^{s}\left(x, B_{i}\right) \subset \mathscr{T}^{s}\left(B_{i}, B_{j}\right) \\
& y \in \mathscr{T}^{u}\left(B_{i}, B_{j}\right) \Rightarrow W^{u}\left(y, B_{j}\right) \subset \mathscr{T}^{u}\left(B_{i}, B_{j}\right) .
\end{aligned}
$$

Above, $W^{s}\left(x, B_{i}\right)=\left\{[x, y]: y \in B_{i}\right\}$ is the intersection of the local stable manifold of $f$ at $x$ with $B_{i}$. The definition of $W^{u}\left(y, B_{j}\right)$ is similar.

5.2. Markov partitions/sections generate symbolic models. If $\mathscr{R}$ is a Markov partition for $f$, then $f$ has a symbolic model defined by:

$\circ \mathscr{G}=(V, E)$ with $V=\mathscr{R}$ and $E=\left\{R \rightarrow S: f\left(R^{*}\right) \cap S^{*} \neq \varnothing\right\}$.

○ $\pi: \Sigma \rightarrow \Omega(f)$ is defined for $\underline{R}=\left\{R_{n}\right\}_{n \in \mathbb{Z}} \in \Sigma$ by

$$
\{\pi(\underline{R})\}:=\bigcap_{n \geq 0} f^{n}\left(R_{-n}\right) \cap \cdots \cap f^{-n}\left(R_{n}\right)=\bigcap_{n \geq 0} \overline{f^{n}\left(R_{-n}\right) \cap \cdots \cap f^{-n}\left(R_{n}\right)} .
$$

Alternatively, $\pi(\underline{R})$ is the unique $x \in \Omega(f)$ s.t. $f^{n}(x) \in R_{n}, \forall n \in \mathbb{Z}$. The map $\pi$ is well-defined due to the Markov property and uniform hyperbolicity. Clearly $f \circ \pi=\pi \circ \sigma$. Additionally, $\pi$ is a finite-to-one continuous surjection that is one-to-one on a residual subset of $\Omega(f)$, see Bow08, Thm. 3.18] for details.

If $\mathscr{M}$ is a Markov section for $\varphi$, then $\varphi$ has a symbolic model:

○ $\mathscr{G}=(V, E)$ with $V=\mathscr{M}$ and $E=\left\{B_{i} \rightarrow B_{j}: \exists x \in \mathscr{M}^{\prime}\right.$ s.t. $\left.x \in B_{i}^{*}, f(x) \in B_{j}^{*}\right\}$.

$\circ \pi: \Sigma \rightarrow \mathscr{M}$ is defined for $\underline{B}=\left\{B_{n}\right\}_{n \in \mathbb{Z}} \in \Sigma$ by

$$
\{\pi(\underline{B})\}:=\bigcap_{n \geq 0} f^{n}\left(B_{-n}\right) \cap \cdots \cap f^{-n}\left(B_{n}\right)=\bigcap_{n \geq 0} \overline{f^{n}\left(B_{-n}\right) \cap \cdots \cap f^{-n}\left(B_{n}\right)} .
$$

$\circ r: \Sigma \rightarrow \mathbb{R}$ is defined by $r:=\mathfrak{t} \circ \pi$.

- $\pi_{r}: \Sigma_{r} \rightarrow \Omega(\varphi)$ is defined by $\pi_{r}(\underline{B}, t):=\varphi^{t}[\pi(\underline{B})]$.

Again, $\pi$ is well-defined because of the Markov property and uniform hyperbolicity, and satisfies $f \circ \pi=\pi \circ \sigma$. Also, $\pi$ is a finite-to-one continuous surjection that is one-to-one on $\mathscr{M}^{\prime}$, see Bow73 for details.

Therefore, to get symbolic models for uniformly hyperbolic symbolic systems, it is enough to construct Markov partitions/sections.

5.3. Markov partitions for two-dimensional hyperbolic toral automorphisms. This method, developed by Adler and Weiss AW67, constructs finite Markov partitions for two-dimensional hyperbolic toral automorphisms. A particular case was constructed by Berg Ber68. Consider the cat map introduced in Example 1 of Subsection 1.1 and let $\overrightarrow{0}=(0,0) \in \mathbb{T}^{2}$. Clearly, $f(\overrightarrow{0})=\overrightarrow{0}$. Since the matrix $A$ is hyperbolic, 0 has two eigendirections, call $W^{s}$ the contracting one and $W^{u}$ the expanding one. By linearity, $W^{s}$ and $W^{u}$ are the (global) stable and unstable manifolds of $\overrightarrow{0}$.

The idea to obtain a Markov partition is to construct a fundamental domain of $\mathbb{T}^{2}$ whose sides are pieces of $W^{s}$ and $W^{u}$, and then subdivide this domain into finitely many rectangles satisfying the Markov property. In Figure 12, we draw one possibility for the tesselation of $\mathbb{R}^{2}$ by one such fundamental domain. For a 


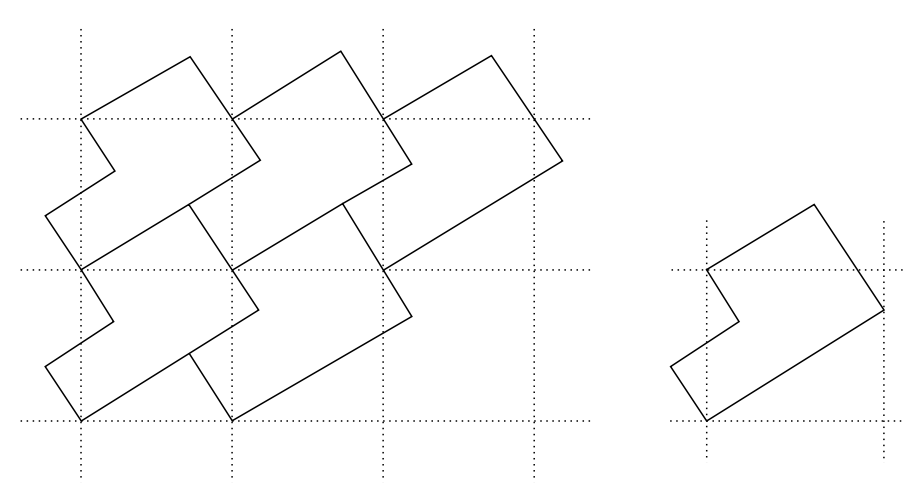

Figure 12. A tesselation of $\mathbb{R}^{2}$ by fundamental domains whose sides are parallel to $W^{s}$ and $W^{u}$.

general two-dimensional hyperbolic toral automorphism, the construction of the fundamental domain consists of two steps.

STEP 1 . Take a cover $\mathscr{R}$ of $\mathbb{T}^{2}$ by finitely many rectangles whose sides belong to $W^{s}$ and $W^{u}$ s.t. that every non-trivial intersection $f\left(R^{*}\right) \cap S^{*}$ is connected, i.e. $f\left(R^{*}\right)$ does not intersect $S^{*}$ "twice".

SteP 2. Since $f$ contracts $W^{s}$, which is the stable manifold of the fixed point $\overrightarrow{0}$, the stable boundary of $f(\mathscr{R})$ is contained in $W^{s}$, while its unstable boundary contains $W^{u}$. Partition $\mathscr{R}$ further by adding the pre-image of the unstable segments of $f(\mathscr{R})$.

The final cover $\mathscr{R}$ is a finite Markov partition, see AW70 for details. The projection map $\pi: \Sigma \rightarrow \mathbb{T}^{2}$ is a finite-to-one continuous surjection that is one-toone on the set $\left\{x \in \mathbb{T}^{2}: f^{n}(x) \in \bigcup_{R \in \mathscr{R}} R^{*}, \forall n \in \mathbb{Z}\right\}$.

In our example, it is enough to divide the fundamental domain into three rectangles $R_{1}, R_{2}, R_{3}$ as in Figure 13 . We leave as exercise to show that the images $f\left(R_{1}\right), f\left(R_{2}\right), f\left(R_{3}\right)$ are as depicted in Figure 13 , so that $\left\{R_{1}, R_{2}, R_{3}\right\}$ is a Markov partition. The graph defining the TMS is also depicted in Figure 13

For higher dimensional hyperbolic toral automorphisms, a similar construction works, but there is an important difference from the two-dimensional case: the boundary of a Markov partition is not smooth Bow78a.

5.4. The method of successive approximations for diffeomorphisms. This method, due to Sinar [Sin68b, Sin68a, provides Markov partitions for Anosov diffeomorphisms. It was later modified by Bowen to also work for Axiom A diffeomorphisms Bow70a]. The construction consists of three main steps. Below, we explain them for Anosov diffeomorphisms.

Step 1 (CoArse graining). Let $\mathscr{T}=\left\{T_{i}\right\}$ be a finite cover of $M$ by rectangles (as we have argued in Subsection 5.1. it is easy to build one such cover).

Step 2 (Successive Approximations). Recursively define families $\mathscr{I}_{k}=\left\{S_{i, k}\right\}$ and $\mathscr{U}_{k}=\left\{U_{i, k}\right\}$ of rectangles as follows:

○ $S_{i, 0}=U_{i, 0}=T_{i}$. 

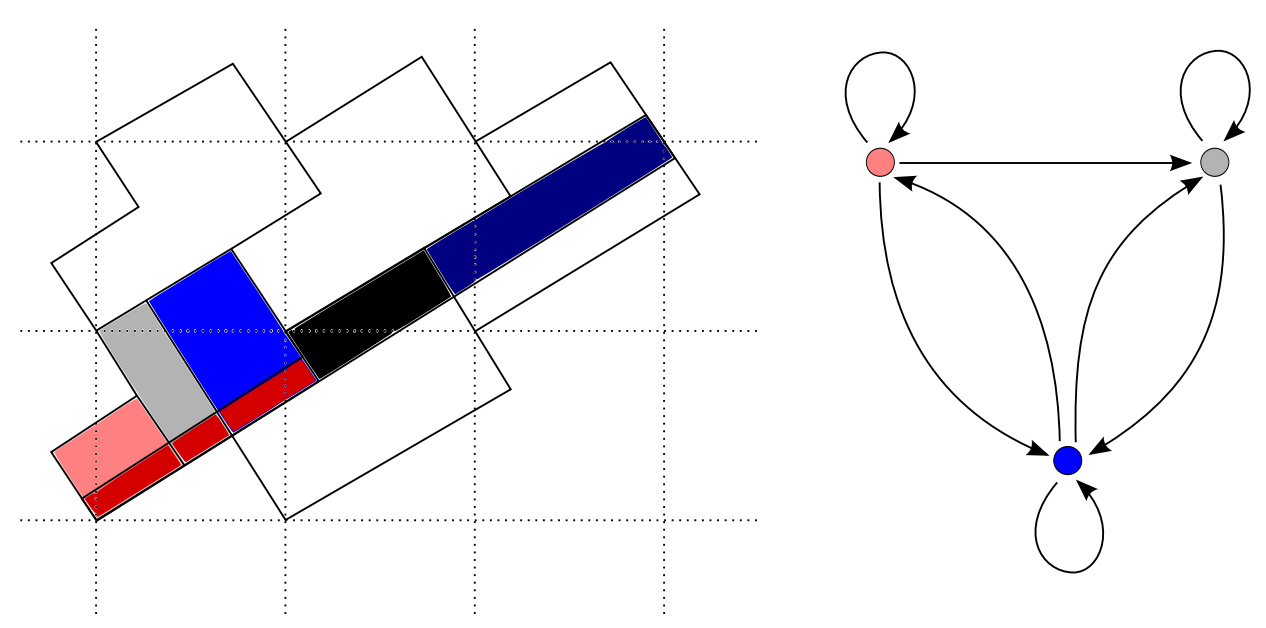

Figure 13. A Markov partition for the cat map by three rectangles $R_{1}, R_{2}, R_{3}$, and the graph defining the respective TMS.

○ If $\mathscr{S}_{k}, \mathscr{U}_{k}$ are defined, let

$$
\begin{aligned}
S_{i, k+1} & :=\bigcup_{x \in S_{i, k}}\left\{[y, z]: y \in S_{i, k}, z \in f\left(W^{s}\left(f^{-1}(x), S_{j, k}\right)\right) \text { for } f^{-1}(x) \in S_{j, k}\right\} \\
U_{i, k+1} & :=\bigcup_{x \in U_{i, k}}\left\{[z, y]: y \in U_{i, k}, z \in f^{-1}\left(W^{u}\left(f(x), U_{j, k}\right)\right) \text { for } f(x) \in U_{j, k}\right\} .
\end{aligned}
$$

Let $S_{i}:=\bigcup_{k \geq 0} S_{i, k}, U_{i}:=\bigcup_{k \geq 0} U_{i, k}$, and $Z_{i}:=\left[\overline{U_{i}}, \overline{S_{i}}\right]$. Then $\mathscr{Z}=\left\{Z_{i}\right\}$ is a Markov cover.

Let us understand the above definitions. Representing the stable direction by the horizontal direction, we identify what are the horizontal components of $S_{i, k+1} \backslash S_{i, k}$, see Figure 14. Start observing that the horizontal component of $S_{i, 1} \backslash S_{i, 0}$ is the
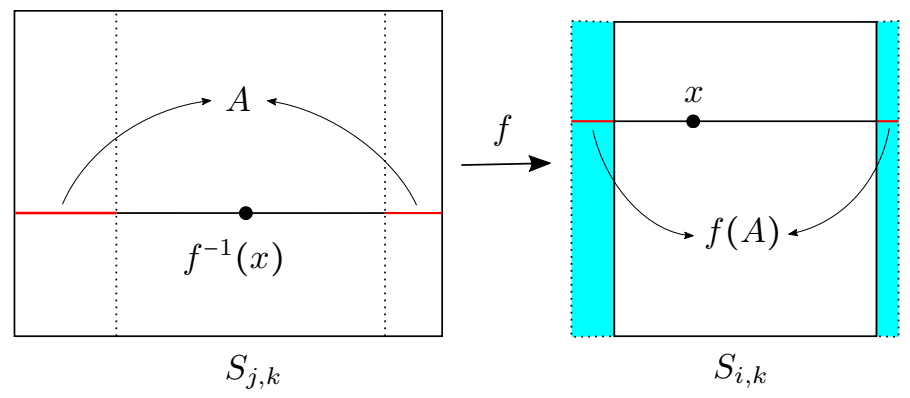

Figure 14. The horizontal component of $S_{i, k+1} \backslash S_{i, k}$ is the union of sets of the form $f(A)$, where $A$ is a horizontal subset of $S_{j, k} \backslash S_{j, k-1}$. Above, $A$ is composed of the two ticker segments on the left figure and the added set is in grey in the right figure.

union of sets of the form $f(A)$, where $A=W^{s}\left(f^{-1}(x), S_{j, 0}\right) \backslash f^{-1}\left(W^{s}\left(x, S_{i, 0}\right)\right)$. Each such $A$ has diameter less than 1 , hence $f(A)$ has diameter less than $\kappa$, thus $S_{i, 1}$ equals the union of $S_{i, 0}$ and a set of horizontal diameter less than $\kappa$. Similarly, the 
horizontal component of $S_{i, 2} \backslash S_{i, 1}$ is the union of sets of the form $f(A)$, where $A$ is a horizontal subset of $S_{j, 1} \backslash S_{j, 0}$, therefore $S_{i, 2}$ equals the union of $S_{i, 1}$ and a set of horizontal diameter less than $\kappa^{2}$. By induction, $S_{i, k+1}$ equals the union of $S_{i, k}$ and a set of horizontal diameter less than $\kappa^{k+1}$. This shows that each $S_{i}$ is well-defined, and the same occurs for each $U_{i}$.

Step 3 (Bowen-SinaĬ REFInEment). To destroy non-trivial intersections, refine $\mathscr{Z}$ as follows. For $Z_{i}$, let $I_{i}=\left\{j: Z_{i}^{*} \cap Z_{j}^{*} \neq \varnothing\right\}$. For $j \in I_{i}$, let $\mathscr{E}_{i j}=$ cover of $Z_{i}$ by rectangles (see Figure 15):

$$
\begin{aligned}
& E_{i j}^{s u}:=\overline{\left\{x \in Z_{i}^{*}: W^{s}\left(x, Z_{i}\right) \cap Z_{j}^{*} \neq \varnothing, W^{u}\left(x, Z_{i}\right) \cap Z_{j}^{*} \neq \varnothing\right\}} \\
& E_{i j}^{s \varnothing}:=\overline{\left\{x \in Z_{i}^{*}: W^{s}\left(x, Z_{i}\right) \cap Z_{j}^{*} \neq \varnothing, W^{u}\left(x, Z_{i}\right) \cap Z_{j}=\varnothing\right\}} \\
& E_{i j}^{\varnothing u}:=\overline{\left\{x \in Z_{i}^{*}: W^{s}\left(x, Z_{i}\right) \cap Z_{j}=\varnothing, W^{u}\left(x, Z_{i}\right) \cap Z_{j}^{*} \neq \varnothing\right\}} \\
& E_{i j}^{\varnothing \varnothing}:=\overline{\left\{x \in Z_{i}^{*}: W^{s}\left(x, Z_{i}\right) \cap Z_{j}=\varnothing, W^{u}\left(x, Z_{i}\right) \cap Z_{j}=\varnothing\right\} .} .
\end{aligned}
$$

Hence $\mathscr{R}:=$ cover defined by $\left\{\mathscr{E}_{i j}: Z_{i} \in \mathscr{Z}, j \in I_{i}\right\}$ is a Markov partition for $f$, and

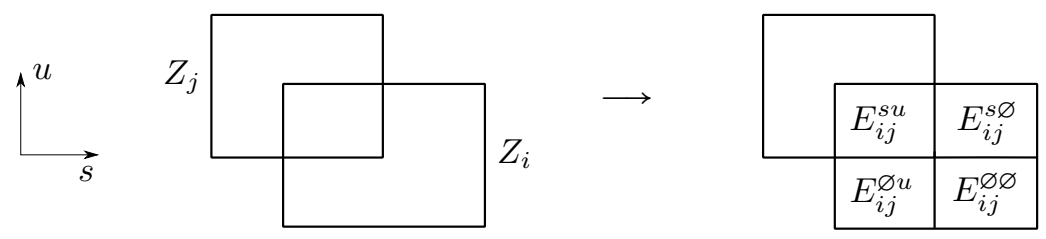

FiguRE 15. $\mathscr{E}_{i j}=\left\{E_{i j}^{s u}, E_{i j}^{s \varnothing}, E_{i j}^{\varnothing u}, E_{i j}^{\varnothing \varnothing}\right\}$ is a cover of $Z_{i}$ by rectangles.

the induced $\pi: \Sigma \rightarrow M$ is a finite-to-one continuous surjection that is one-to-one on $\left\{x \in M: f^{n}(x) \in \cup_{R \in \mathscr{R}} R^{*}, \forall n \in \mathbb{Z}\right\}$.

5.5. The method of successive approximations for flows. Ratner applied the method of successive approximations for three dimensional Anosov flows Rat69. Later she extended it for higher dimensional Anosov flows [Rat73], and Bowen used it for Axiom A flows Bow73]. Below, we follow Bowen's construction. As usual, the main difficulty when dealing with flows is the presence of discontinuities for the Poincaré return map.

Consider a proper section $\mathscr{C}$. Since the stable/unstable directions of $\varphi$ project to stable/unstable directions of the Poincaré map $f$, it is easy to construct rectangles inside $\mathscr{C}$. Let $\mathscr{R}$ be a cover of $\mathscr{C} \cap \Omega(\varphi)$ by rectangles. To apply successive approximations (Step 2 of the last subsection), proceed as follows:

○ Take $L>0$ large s.t. for every $x \in R \in \mathscr{R}$ there are $C^{+}, C^{-} \in \mathscr{C}$ s.t. $\varphi^{L}\left(W_{\text {loc }}^{s}(x)\right) \subset$ $\varphi^{[-\alpha, \alpha]}\left(C^{+}\right)$and $\varphi^{-L}\left(W_{\mathrm{loc}}^{u}(x)\right) \subset \varphi^{[-\alpha, \alpha]}\left(C^{-}\right)$. The existence of $L$ follows from the uniform hyperbolicity of $\varphi$.

- For each such $x$, take a neighborhood $V \ni x$ small enough s.t. $\varphi^{L}(V) \subset \varphi^{[-\alpha, \alpha]}\left(C^{+}\right)$ and $\varphi^{-L}(V) \subset \varphi^{[-\alpha, \alpha]}\left(C^{-}\right)$, and define $f_{V}^{+}: V \rightarrow C^{+}$and $f_{V}^{-}: V \rightarrow C^{-}$by:

$$
f_{V}^{+}:=\left(\text {projection to } C^{+}\right) \circ \varphi^{L}, f_{V}^{-}:=\left(\text {projection to } C^{-}\right) \circ \varphi^{-L} \text {. }
$$

- Pass to a finite collection of neighborhoods $V$ as above, and apply the method of successive approximations to the maps $f_{V}^{+}, f_{V}^{-}$. The resulting cover by rectangles has a Markov property: for each $x \in \mathscr{R}$ there are $k, \ell>0$ s.t. $x$ satisfies a stable Markov property at $f^{k}(x)$ and an unstable Markov property at $f^{-\ell}(x)$. 
- The values of $k, \ell$ are uniformly bounded by some $N>0$.

- To get the Markov property for $f$, apply a refinement procedure along the iterates $-N, \ldots, N$ of $f$. The resulting partition $\mathscr{M}$ is a Markov section for $\varphi$.

See the details in Bow73.

5.6. The method of pseudo-orbits. Bowen provided an alternative method to construct Markov partitions for Axiom A diffeomorphisms Bow08. His idea was to use the theory of pseudo-orbits and shadowing, which explores the expected richness on the orbit structure of uniformly hyperbolic systems. These notions appeared in the qualitative theory of structural stability for uniformly hyperbolic systems. Indeed, Anosov considered a version of pseudo-orbits for flows, which he called $\varepsilon$-trajectories, and used them to prove that Anosov flows are structurally stable, see Ano70, Thm. 1].

Let $f: M \rightarrow M$ be an invertible map. An orbit of $f$ is a sequence $\left\{x_{n}\right\}_{n \in \mathbb{Z}}$ s.t. $f\left(x_{n}\right)=x_{n+1}$ for all $n \in \mathbb{Z}$, while a pseudo-orbit is a sequence $\left\{x_{n}\right\}_{n \in \mathbb{Z}}$ s.t. $f\left(x_{n}\right) \approx x_{n+1}$ for all $n \in \mathbb{Z}$. In other words, a pseudo-orbit is an orbit up to small errors at each iteration. This is exactly what a computer returns when we try to iterate a map: due to roundoff errors, the sequence is not a real orbit but just a pseudo-orbit. Since a hyperbolic matrix remains hyperbolic after a small perturbation, Theorem 1.2 holds for pseudo-orbits, as we will see below: changing $f(x)$ to some nearby $y$, we can represent $f$ in the Lyapunov charts $\Psi_{x}$ and $\Psi_{y}$ and still obtain a small perturbation of a hyperbolic matrix. This is the main tool to introduce the symbolic model. To maintain consistency with Part 1 , we continue assuming that $M$ is a surface.

5.6.1. Pseudo-orbits. Recall from Section 1.5 the definition of Lyapunov charts: for $\varepsilon>0$ is small enough we let $Q=\varepsilon^{3 / \beta}$ and define, for each $x \in M$, its Lyapunov chart $\Psi_{x}:[-Q, Q]^{2} \rightarrow M$. Recall that $\Psi_{x}$ is 2 -Lipschitz and its inverse is $2 \mathscr{L}$-Lipschitz. The splitting $E^{s} \oplus E^{u}$ is continuous, so there is $\delta=\delta(\varepsilon)>0$ s.t. if $d(x, y)<\delta$ then $\left\|\Psi_{y}^{-1} \circ \Psi_{x}-\mathrm{Id}\right\|_{1+\beta / 2}<\varepsilon^{3}$, where the norm is taken in $[-Q, Q]^{2}{ }^{8}$

$\varepsilon$-OVERlaP: Two Lyapunov charts $\Psi_{x}, \Psi_{y}$ are said to $\varepsilon$-overlap if $d(x, y)<\delta$. When this happens, we write $\Psi_{x} \stackrel{\varepsilon}{\approx} \Psi_{y}$.

Hence, if two points are close enough, the charts they define are essentially the same. This notation is somewhat redundant for uniformly hyperbolic systems, but we prefer to state it as above because it helps understanding the symbolic model and the difficulties when we consider nonuniformly hyperbolic systems.

If $\Psi_{f(x)} \stackrel{\varepsilon}{\approx} \Psi_{y}$, then we can write $f$ in the Lyapunov charts $\Psi_{x}$ and $\Psi_{y}$ as $f_{x, y}=\Psi_{y}^{-1} \circ f \circ \Psi_{x}$. Since $f_{x, y}=\Psi_{y}^{-1} \circ \Psi_{f(x)} \circ f_{x}=: g \circ f_{x}$, where $g:=\Psi_{y}^{-1} \circ \Psi_{f(x)}$ is a small perturbation of the identity, the map $f_{x, y}$ is again a small perturbation of a hyperbolic matrix. The same reasoning happens if $\Psi_{f^{-1}(y)} \underset{\approx}{\approx} \Psi_{x}$, in which case $f_{x, y}^{-1}=\Psi_{x}^{-1} \circ f^{-1} \circ \Psi_{y}$, the representation of $f^{-1}$ in the Lyapunov charts $\Psi_{x}$ and $\Psi_{y}$, is also a small perturbation of a hyperbolic matrix. This is summarized in the next theorem, which is the version of Theorem 1.2 in the present context.

\footnotetext{
${ }^{8}$ The composition $\Psi_{y}^{-1} \circ \Psi_{x}$ is well-defined in $[-Q, Q]^{2}$. To see this, fix $\varepsilon>0$ small enough so that each $\Psi_{x}$ is well-defined in the larger domain $[-10 \mathscr{L} Q, 10 \mathscr{L} Q]^{2}$. Taking $\delta=\delta(\varepsilon)>0$ small enough, if $d(x, y)<\delta$ then $\Psi_{x}\left([-Q, Q]^{2}\right) \subset B(x, 4 Q) \subset B(y, 5 Q) \subset \Psi_{y}\left([-10 \mathscr{L} Q, 10 \mathscr{L} Q]^{2}\right)$.
} 
Theorem 5.1. The following hold for all $\varepsilon>0$ small enough. If $\Psi_{f(x)} \stackrel{\varepsilon}{\approx} \Psi_{y}$, then $f_{x, y}$ is well-defined in $[-Q, Q]^{2}$ and can be written as $f_{x, y}\left(v_{1}, v_{2}\right)=\left(A v_{1}+\right.$ $\left.h_{1}\left(v_{1}, v_{2}\right), B v_{2}+h_{2}\left(v_{1}, v_{2}\right)\right)$ where:

(1) $|A|,\left|B^{-1}\right|<\lambda$, cf. Lemma 1.1 .

(2) $\left\|h_{i}\right\|_{1+\beta / 3}<\varepsilon$ for $i=1,2$.

If $\Psi_{f^{-1}(y)} \stackrel{\varepsilon}{\approx} \Psi_{x}$ then a similar statement holds for $f_{x, y}^{-1}$.

Observe that, in contrast to Theorem 1.2 above we can only control the $C^{1+\beta / 3}$ norm. This slight decrease is necessary to keep the estimates of size $\varepsilon$.

EDGE: We write $\Psi_{x} \stackrel{\varepsilon}{\rightarrow} \Psi_{y}$ if $\Psi_{f(x)} \stackrel{\varepsilon}{\approx} \Psi_{y}$ and $\Psi_{f^{-1}(y)} \stackrel{\varepsilon}{\approx} \Psi_{x}$.

Conditions $\Psi_{f(x)} \stackrel{\varepsilon}{\approx} \Psi_{y}$ and $\Psi_{f^{-1}(y)} \stackrel{\varepsilon}{\approx} \Psi_{x}$ are called nearest neighbor conditions.

PSEUdo-ORBIT: A sequence of Lyapunov charts $\left\{\Psi_{x_{n}}\right\}_{n \in \mathbb{Z}}$ is called a pseudo-orbit if $\Psi_{x_{n}} \stackrel{\varepsilon}{\rightarrow} \Psi_{x_{n+1}}$ for all $n \in \mathbb{Z}$.

We point out that classically a pseudo-orbit is a sequence of points instead of Lyapunov charts, but so far these notions coincide, since $\Psi_{x_{n}} \stackrel{\varepsilon}{\rightarrow} \Psi_{x_{n+1}}$ is equivalent to $d\left(f\left(x_{n}\right), x_{n+1}\right)<\delta$ and $d\left(f^{-1}\left(x_{n+1}\right), x_{n}\right)<\delta$.

5.6.2. Graph transforms. Assume that $\Psi_{x} \stackrel{\varepsilon}{\rightarrow} \Psi_{y}$. Since $f_{x y}^{ \pm 1}$ are perturbations of hyperbolic matrices, we can proceed as in Subsection 1.6 and define graph transforms between admissible manifolds at $\Psi_{x}$ and $\Psi_{y}$. First, we need to redefine admissibility. For instance, we can no longer require $F(0)=0$, since this property is not preserved by the maps $f_{x, y}^{ \pm 1}$ (unless $f(x)=y$ ). For ease of exposition, we continue not prescribing the precision in this definition.

Admissible MANIfolds: An $s$-admissible manifold at $\Psi_{x}$ is a set of the form $V^{s}=\Psi_{x}\{(t, F(t)):|t| \leq Q\}$, where $F:[-Q, Q] \rightarrow \mathbb{R}$ is a $C^{1}$ function s.t. $F(0) \approx 0$ and $\left\|F^{\prime}\right\|_{C^{0}} \approx 0$. Similarly, a $u$-admissible manifold at $\Psi_{x}$ is a set of the form $V^{u}=\Psi_{x}\{(G(t), t):|t| \leq Q\}$, where $G:[-Q, Q] \rightarrow \mathbb{R}$ is a $C^{1}$ function s.t. $G(0) \approx 0$ and $\left\|G^{\prime}\right\|_{C^{0}} \approx 0$.

Let $\mathscr{M}_{x}^{s}, \mathscr{M}_{x}^{u}$ be the space of all $s, u$-admissible manifolds at $\Psi_{x}$ respectively, and introduce metrics on $\mathscr{M}_{x}^{s / u}$ as before. Assume that $\Psi_{x} \stackrel{\varepsilon}{\rightarrow} \Psi_{y}$.

GRAPH TRANSFORMS $\mathscr{F}_{x, y}^{s}, \mathscr{F}_{x, y}^{u}$ : The stable graph transform $\mathscr{F}_{x, y}^{s}: \mathscr{M}_{y}^{s} \rightarrow \mathscr{M}_{x}^{s}$ is the map that sends $V^{s} \in \mathscr{M}_{y}^{s}$ to the unique $\mathscr{F}_{x, y}^{s}\left[V^{s}\right] \in \mathscr{M}_{x}^{s}$ with representing function $F$ s.t. $\Psi_{x}\{(t, F(t)):|t| \leq Q\} \subset f^{-1}\left(V^{s}\right)$. Similarly, the unstable graph transform $\mathscr{F}_{x, y}^{u}: \mathscr{M}_{x}^{u} \rightarrow \mathscr{M}_{y}^{u}$ is the map that sends $V^{u} \in \mathscr{M}_{x}^{u}$ to the unique $\mathscr{F}_{x, y}^{u}\left[V^{u}\right] \in \mathscr{M}_{y}^{u}$ with representing function $G$ s.t. $\Psi_{y}\{(G(t), t):|t| \leq Q\} \subset f\left(V^{u}\right)$.

Again, the hyperbolicity of $f_{x, y}^{ \pm 1}$ implies the following result.

Theorem 5.2. If $\Psi_{x} \stackrel{\varepsilon}{\rightarrow} \Psi_{y}$, then $\mathscr{F}_{x, y}^{s}$ and $\mathscr{F}_{x, y}^{u}$ are well-defined contractions.

Consequently, each pseudo-orbit has local stable and unstable manifolds.

Stable/Unstable Manifolds: The stable manifold of $\underline{v}=\left\{\Psi_{x_{n}}\right\}_{n \in \mathbb{Z}}$ is the unique $s$-admissible manifold $V^{s}[\underline{v}] \in \mathscr{M}_{x_{0}}^{s}$ defined by

$$
V^{s}[\underline{v}]:=\lim _{n \rightarrow \infty}\left(\mathscr{F}_{x_{0}, x_{1}}^{s} \circ \cdots \circ \mathscr{F}_{x_{n-1}, x_{n}}^{s}\right)\left[V_{n}\right]
$$


for some (any) sequence $\left\{V_{n}\right\}_{n \geq 0}$ with $V_{n} \in \mathscr{M}_{x_{n}}^{s}$. The unstable manifold of $\underline{v}$ is the unique $u$-admissible manifold $V^{u}[\underline{v}] \in \mathscr{M}_{x_{0}}^{u}$ defined by

$$
V^{u}[\underline{v}]:=\lim _{n \rightarrow-\infty}\left(\mathscr{F}_{x_{-1}, x_{0}}^{u} \circ \cdots \circ \mathscr{F}_{x_{n}, x_{n+1}}^{u}\right)\left[V_{n}\right]
$$

for some (any) sequence $\left\{V_{n}\right\}_{n \leq 0}$ with $V_{n} \in \mathscr{M}_{x_{n}}^{u}$.

The observations at the end of Subsection 1.6 can be repeated ipsis literis. In particular, $V^{s}[\underline{v}]$ only depends on the future $\left\{\Psi_{x_{n}}\right\}_{n \geq 0}$, while $V^{u}[\underline{v}]$ only depends on the past $\left\{\Psi_{x_{n}}\right\}_{n \leq 0}$.

SHADOWING: $\underline{v}=\left\{\Psi_{x_{n}}\right\}_{n \in \mathbb{Z}}$ is said to shadow $x$ if $f^{n}(x) \in \Psi_{x_{n}}\left([-Q, Q]^{2}\right), \forall n \in \mathbb{Z}$.

Theorem 5.3 (Shadowing Lemma). Every pseudo-orbit $\underline{v}$ shadows a unique point $\{x\}=V^{s}[\underline{v}] \cap V^{u}[\underline{v}]$.

This follows from the hyperbolicity of each $f_{x_{n}, x_{n+1}}^{ \pm 1}$.

5.6.3. Construction of a Markov partition. Now we explain how Bowen used the above tools to construct a Markov partition for $f$. The construction involves two codings, the first being usually infinite-to-one and the second finite-to-one. We divide the construction into three steps. Let $f: M \rightarrow M$ be Axiom A, and let $L>1$ be a Lipschitz constant for $f^{ \pm 1}$.

Step 1 (CoArse Graining). Fix a finite subset of $X \subset \Omega(f)$ that is $\frac{\delta}{2 L}$-dense in $\Omega(f)$, and let $\mathscr{A}=\left\{\Psi_{x}: x \in X\right\}$. Let $\mathscr{G}=(V, E)$ be the oriented graph with vertex set $V=\mathscr{A}$ and edge set $E=\left\{\Psi_{x} \stackrel{\mathcal{\varepsilon}}{\rightarrow} \Psi_{y}\right\}$, and let $(\Sigma, \sigma)$ be the TMS defined by $\mathscr{G}$. Observe that an element of $\Sigma$ is a pseudo-orbit.

Step 2 (Infinite-to-One extension). Using the Shadowing Lemma, define a map $\pi: \Sigma \rightarrow \Omega(f)$ by

$$
\{\pi(\underline{v})\}:=V^{s}[\underline{v}] \cap V^{u}[\underline{v}] .
$$

The map $\pi$ has the following properties:

○ $\pi$ is surjective: for every $x \in \Omega(f)$, choose $\left\{x_{n}\right\}_{n \in \mathbb{Z}} \subset X$ s.t. $d\left(f^{n}(x), x_{n}\right)<\frac{\delta}{2 L}$.

Then $\underline{v}=\left\{\Psi_{x_{n}}\right\}_{n \in \mathbb{Z}}$ is a pseudo-orbit, since

$$
\begin{aligned}
& d\left(f\left(x_{n}\right), x_{n+1}\right) \leq d\left(f\left(x_{n}\right), f^{n+1}(x)\right)+d\left(f^{n+1}(x), x_{n+1}\right) \\
& \leq L d\left(x_{n}, f^{n}(x)\right)+d\left(f^{n+1}(x), x_{n+1}\right)<\frac{\delta}{2}+\frac{\delta}{2 L}<\delta,
\end{aligned}
$$

and similarly $d\left(f^{-1}\left(x_{n+1}\right), x_{n}\right)<\delta$. Clearly, $\pi(\underline{v})=x$.

$\circ \pi \circ \sigma=f \circ \pi$ : this follows from the Shadowing Lemma, since if $\underline{v}$ shadows $x$ then $\sigma(\underline{v})$ shadows $f(x)$.

- $\pi$ is usually infinite-to-one: imagine, for example, that for some $x \in \Omega(f)$ there are $x_{n}, y_{n} \in X$ s.t. $d\left(f^{n}(x), x_{n}\right)<\frac{\delta}{2 L}$ and $d\left(f^{n}(x), y_{n}\right)<\frac{\delta}{2 L}$. Any choice of $z_{n} \in\left\{x_{n}, y_{n}\right\}$ defines a pseudo-orbit $\left\{\Psi_{z_{n}}\right\}_{n \in \mathbb{Z}}$ that shadows $x$, hence $\pi^{-1}(x)$ has cardinality at least $2^{\mathbb{Z}}$, which is uncountable.

The third property above is, in general, unavoidable. Hence, $(\Sigma, \sigma, \pi)$ is not a symbolic model for $f$. But the Markov structure of $\Sigma$ induces, via $\pi$, a cover of $\Omega(f)$ satisfying a (symbolic) Markov property.

The Markov cover $\mathscr{Z}:$ Let $\mathscr{Z}:=\{Z(v): v \in \mathscr{A}\}$, where

$$
Z(v):=\left\{\pi(\underline{v}): \underline{v} \in \Sigma \text { and } v_{0}=v\right\} .
$$


In other words, $\mathscr{Z}$ is the family defined by the natural partition of $\Sigma$ into cylinder at the zeroth position. In general, each $Z(v)$ is fractal. Admissible manifolds allow us to define invariant fibres inside each $Z \in \mathscr{Z}$. Let $Z=Z(v)$.

$s / u$-FIBRES IN $\mathscr{Z}$ : Given $x \in Z$, let $W^{s}(x, Z):=V^{s}[\underline{v}] \cap Z$ be the $s$-fibre of $x$ in $Z$ for some (any) $\underline{v}=\left\{v_{n}\right\}_{n \in \mathbb{Z}} \in \Sigma$ s.t. $\pi(\underline{v})=x$ and $v_{0}=v$. Similarly, let $W^{u}(x, Z):=V^{u}[\underline{v}] \cap Z$ be the $u$-fibre of $x$ in $Z$.

Observe that, while $V^{s / u}[\underline{v}]$ are smooth manifolds, $W^{s / u}(x, Z)$ is usually fractal. Below we collect the main properties of $\mathscr{Z}$.

Proposition 5.4. The following holds for all $\varepsilon>0$ small enough.

(1) Covering Property: $\mathscr{Z}$ is a cover of $\Omega(f)$.

(2) Product structure: For every $Z \in \mathscr{Z}$ and every $x, y \in Z$, the intersection $W^{s}(x, Z) \cap W^{u}(y, Z)$ consists of a single point, and this point belongs to $Z$.

(3) Symbolic Markov Property: If $x=\pi(\underline{v})$ with $\underline{v}=\left\{v_{n}\right\}_{n \in \mathbb{Z}} \in \Sigma$, then

$f\left(W^{s}\left(x, Z\left(v_{0}\right)\right)\right) \subset W^{s}\left(f(x), Z\left(v_{1}\right)\right)$ and $f^{-1}\left(W^{u}\left(f(x), Z\left(v_{1}\right)\right)\right) \subset W^{u}\left(x, Z\left(v_{0}\right)\right)$.

Part (1) follows from the surjectivity of $\pi$. To prove part (2), we define a Smale bracket $[\cdot, \cdot]_{Z}$ for each $Z \in \mathscr{Z}$ as follows. Write $Z=Z(v)$, and let $x=\pi(\underline{v}), y=\pi(\underline{w})$ where $\underline{v}=\left\{v_{n}\right\}_{n \in \mathbb{Z}}, \underline{w}=\left\{w_{n}\right\}_{n \in \mathbb{Z}} \in \Sigma$ with $v_{0}=w_{0}=v$. Then $W^{s}(x, Z) \cap W^{u}(y, \bar{Z})$ consists of a unique point $z=\pi(\underline{u})$ where $\underline{u}=\left\{u_{n}\right\}_{n \in \mathbb{Z}}$ is defined by:

$$
u_{n}=\left\{\begin{array}{cl}
v_{n} & , \text { if } n \geq 0 \\
w_{n} & , \text { if } n \leq 0 .
\end{array}\right.
$$

The equality $z=\pi(\underline{u})$ follows from the Shadowing Lemma. Observe that $z \in Z$. We write $z=:[x, y]_{Z}$. Finally, part (3) follows from the Markov structure of $\Sigma$. At this point, it is also important to show that the above definitions are compatible among the elements of $\mathscr{Z}$.

Lemma 5.5. The following holds for all $\varepsilon>0$ small enough.

(1) Compatibility: If $x, y \in Z\left(v_{0}\right)$ and $f(x), f(y) \in Z\left(v_{1}\right)$ with $v_{0} \stackrel{\varepsilon}{\rightarrow} v_{1}$ then $f\left([x, y]_{Z\left(v_{0}\right)}\right)=[f(x), f(y)]_{Z\left(v_{1}\right)}$.

(2) OverlapPing CHARTS PROPERTIEs: If $Z=Z\left(\Psi_{x}\right), Z^{\prime}=Z\left(\Psi_{y}\right) \in \mathscr{Z}$ with $Z \cap Z^{\prime} \neq \varnothing$ then:

(a) $Z \subset \Psi_{y}\left([-Q, Q]^{2}\right)$.

(b) If $x \in Z \cap Z^{\prime}$ then $W^{s / u}(x, Z) \subset V^{s / u}\left(x, Z^{\prime}\right)$.

(c) If $x \in Z, y \in Z^{\prime}$ then $V^{s}(x, Z)$ and $V^{u}\left(y, Z^{\prime}\right)$ intersect at a unique point.

Part (1) also follows from the Markov structure of $\Sigma$, while part (2) follows from the fine control we have on the Lyapunov charts inside each rectangle $[-Q, Q]^{2}$. Lemma 5.5 allows us to consider Smale brackets of different intersecting rectangles.

Step 3 (Bowen-SinA I REFInEMENT). We repeat ipsis literis Step 3 performed in Subsection 5.4. The resulting partition $\mathscr{R}$ is a Markov partition. By Subsection 5.2 , we obtain a symbolic model $(\widehat{\Sigma}, \widehat{\sigma}, \widehat{\pi})$, where $(\widehat{\Sigma}, \widehat{\sigma})$ is the TMS defined by the graph $\widehat{\mathscr{G}}=(\widehat{V}, \widehat{E})$ with vertex set $\widehat{V}=\mathscr{R}$ and edge set $\widehat{E}=\left\{R \rightarrow S: f\left(R^{*}\right) \cap S^{*} \neq \varnothing\right\}$. Let $\widehat{\pi}: \Sigma \rightarrow \Omega(f)$ be defined for $\underline{R}=\left\{R_{n}\right\}_{n \in \mathbb{Z}} \in \Sigma$ by

$$
\{\widehat{\pi}(\underline{R})\}:=\bigcap_{n \geq 0} f^{n}\left(R_{-n}\right) \cap \cdots \cap f^{-n}\left(R_{n}\right)=\bigcap_{n \geq 0} \overline{f^{n}\left(R_{-n}\right) \cap \cdots \cap f^{-n}\left(R_{n}\right)} .
$$

The $\widehat{\pi}$ is a finite-to-one surjection that is one-to-one on the set $\left\{x \in \Omega(f): f^{n}(x) \in\right.$ $\left.\cup_{R \in \mathscr{R}} R^{*}, \forall n \in \mathbb{Z}\right\}$. 
Remark 5.6. The method of pseudo-orbits also works for uniformly expanding maps, in which case the TMS is one-sided. To do this, assume for simplicity that $f: M \rightarrow M$ satisfies $d(f(x), f(y)) \geq \kappa^{-1} d(x, y)$. Define Lyapunov charts simply by $\Psi_{x}:=\exp _{x}$, then define an edge $\Psi_{x} \stackrel{\varepsilon}{\rightarrow} \Psi_{y}$ iff $d(f(x), y) \ll 1$, and prove that each pseudo-orbit $\left\{\Psi_{x_{n}}\right\}_{n \geq 0}$ shadows a single point $x \in M$. Now implement Steps 1-3.

5.6.4. Bowen relation. We explain why $\widehat{\pi}$ is finite-to-one. The proof follows Bowen Bow 78b], and is sometimes referred as the diamond argument, as justified by Figure 16. See also Adl98, Lemma 6.7]. Bowen's idea was to investigate the quotient map $\pi: \Sigma \rightarrow M$. For us, this argument will be extremely useful to prove, in Section 6. that the coding obtained for nonuniformly hyperbolic maps is finite-to-one. For uniformly hyperbolic maps, this argument is not essential, but we stress that the method is interesting in its own and introduces a relation, nowadays called Bowen relation, that precisely characterizes the loss of injectivity of $\pi$.

Consider the triple $(\widehat{\Sigma}, \widehat{\sigma}, \widehat{\pi})$ as above. Define a relation in $\mathscr{R}$ by $R \sim S$ iff $R \cap S \neq$ $\varnothing$. Assume that $R \sim S$. If $x \in R$ and $y \in S$, let $[x, y]$ be their Smale bracket, which is well-defined by part (2)(c) of Lemma 5.5. Let $\underline{R}=\left\{R_{n}\right\}_{n \in \mathbb{Z}}, \underline{S}=\left\{S_{n}\right\}_{n \in \mathbb{Z}} \in \Sigma$.

Bowen Relation: We say that $\underline{R} \approx \underline{S}$ if $R_{n} \sim S_{n}$ for all $n \in \mathbb{Z}$.

This clearly defines an equivalence relation on $\Sigma$, with $\widehat{\pi}(\underline{R})=\widehat{\pi}(\underline{S})$ iff $\underline{R} \approx \underline{S}$. Let $N=\# \mathscr{R}$.

Lemma 5.7. The following holds for all $\varepsilon>0$ small enough.

(1) If $R_{n} \rightarrow \cdots \rightarrow R_{m}$ and $S_{n} \rightarrow \cdots \rightarrow S_{m}$ are paths on $\widehat{\mathscr{G}}$ s.t. $R_{n}=S_{n}, R_{m}=S_{m}$ and $R_{k} \sim S_{k}$ for $k=n, \ldots, m$, then $R_{k}=S_{k}$ for $k=n, \ldots, m$.

(2) $\widehat{\pi}$ is everywhere at most $N^{2}$-to-one, i.e. for every $x$ we have $\# \widehat{\pi}^{-1}(x) \leq N^{2}$.

Proof. The original reference is Bow78b, pp. 13-14]. As mentioned by Bowen himself, part (2) was pointed out by Brian Marcus. Write $A=R_{n}=S_{n}$ and $B=R_{m}=S_{m}$. For part (1), choose $x, y$ s.t. $f^{k}(x) \in R_{k}^{*}$ and $f^{k}(y) \in S_{k}^{*}$ for $k=n, \ldots, m$. Define $z$ by the equality $f^{n}(z)=\left[f^{n}(x), f^{n}(y)\right]$. Since $R_{k} \sim S_{k}$, we have $f^{k}(z)=\left[f^{k}(x), f^{k}(y)\right]$ for $k=n, \ldots, m$. Noting that $f^{n}(x), f^{n}(y) \in A^{*}$ and $f^{m}(x), f^{m}(y) \in B^{*}$, we have that $f^{n}(z) \in A^{*}$ and $f^{m}(z) \in B^{*}$. Now we use the Markov property:

- The Markov property for the stable direction at $f^{n}(x) \in A$ implies that $f^{k}(z) \epsilon$ $W^{s}\left(f^{k}(x), R_{k}\right)$ for $k=n, \ldots, m$. Indeed, we can prove inductively that $f^{k}(z) \epsilon$ $\left(W^{s}\left(f^{k}(x), R_{k}\right)\right)^{*}$, the interior of $W^{s}\left(f^{k}(x), R_{k}\right)$ in the relative topology of $R_{k}$. In particular, $f^{k}(z) \in R_{k}^{*}$ for $k=n, \ldots, m$.

- Applying the same argument for the unstable direction of $f^{m}(y) \in B$, we obtain that $f^{k}(z) \in S_{k}^{*}$ for $k=n, \ldots, m$.

Hence $f^{k}(z) \in R_{k}^{*} \cap S_{k}^{*}$ and so $R_{k}=S_{k}$, which proves (1). Now we prove (2). If some $x$ has more than $N^{2}$ pre-images, then there are two of them, say $\underline{R}$ and $\underline{S}$, and two indices $n<m$ s.t. $\left(R_{n}, \ldots, R_{m}\right) \neq\left(S_{n}, \ldots, S_{m}\right)$ with $R_{n}=S_{n}$ and $R_{m}=S_{m}$. Since $f^{k}(x) \in R_{k} \cap S_{k}$, we have $R_{k} \sim S_{k}$ for $k=n, \ldots, m$. This contradicts part (1). 


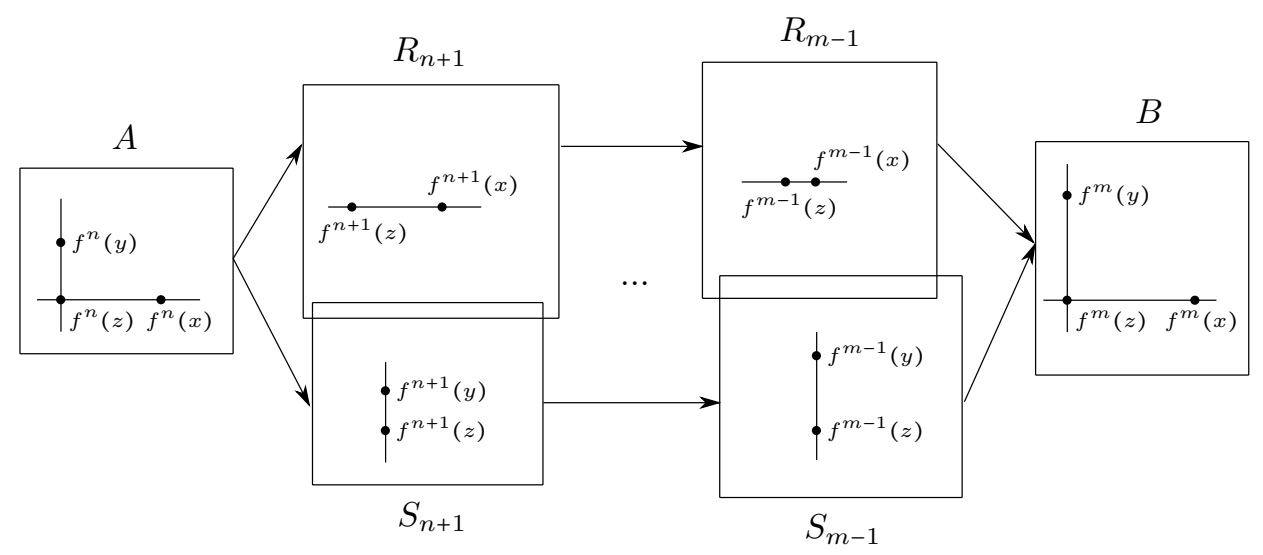

Figure 16. The diamond argument.

\section{SYMBOLIC DYNAMICS FOR NONUNIFORMLY HYPERBOLIC SYSTEMS}

We finally arrive at the core of the discussion, showing how to employ the method of pseudo-orbits for nonuniformly hyperbolic systems. As mentioned in the introduction, Katok was the first to use the theory of pseudo-orbits for hyperbolic measures [Kat80], and constructed the nowadays called Katok horseshoes. Restricted to $C^{1+\beta}$ surface diffeomorphisms, his construction provides finite Markov partitions that approximate the topological entropy. For that, he used Pesin theory on subsets where the parameters vary continuously. This approach is not genuinely nonuniformly hyperbolic, because it discards regions with bad behavior of such parameters. In this section, we explain how this difficulty was solved by Sarig [Sar13]. The starting step is to control the hyperbolicity parameters more effectively, as explained in Section 2. Now, we will use it to develop a finer theory of pseudo-orbits, that in particular provides symbolic models for nonuniformly hyperbolic systems. The idea for construction of the symbolic model is similar to Bowen's method described in Subsection 5.6, but instead of Lyapunov charts we use (double) Pesin charts as vertices of the TMS. In order to code all points with some nonuniform hyperbolicity, invariably we will need countably many such charts. Hence, while for uniformly hyperbolic systems the TMS has finitely many states, now it will have countably many.

In the sequel, we will restrict ourselves to $C^{1+\beta}$ surface diffeomorphisms. Later, we explain how to perform the construction in other settings, which include higher dimensional diffeomorphisms, flows, and billiard maps. We will emphasize five main ingredients in the proof:

o $\varepsilon$-overlap.

- $\varepsilon$-double charts.

- Coarse graining.

- Improvement lemma.

- Inverse theorem.

The first two are discussed in the next subsection, and the others in the subsequent subsections. 
6.1. Preliminaries. Let $M$ be a closed connected smooth Riemannian surface, let $f: M \rightarrow M$ be a $C^{1+\beta}$ diffeomorphism, and fix $\chi>0$. Recall from Subsection 2.2 the definitions of the nonuniformly hyperbolic locus $\mathrm{NUH}_{\chi}$ and Pesin charts $\Psi_{x}$. One important part of the construction will be to restrict the domains of Pesin charts, tuning them properly. Since we want to end up with countably many of them, we choose their sizes from a countable set. Fix $\varepsilon>0$ small enough, and let $I_{\varepsilon}:=\left\{e^{-\frac{1}{3} \varepsilon n}: n \geq 0\right\}$. We redefine $Q(x)$ as below.

Parameter $Q(x)$ : For each $x \in \mathrm{NUH}_{\chi}$, define $Q(x)$ to be the largest element of $I_{\varepsilon}$ that is $\leq \varepsilon^{3 / \beta}\left\|C(f(x))^{-1}\right\|_{\text {Frob }}^{-12 / \beta}$.

In other words, we truncate $Q(x)$ to $I_{\varepsilon}$. Now, define the parameters $q, q^{s}, q^{u}$ and the nonuniformly hyperbolic locus $\mathrm{NUH}_{\chi}^{*}$ as in Subsections 2.5 and 2.6. Observe that $q, q^{s}, q^{u} \in I_{\varepsilon}$. To obtain a finite-to-one coding, we need a recurrence assumption on the parameter $q$, so we define a subset of $\mathrm{NUH}_{\chi}^{*}$ as follows.

The NONUNIFORMLY HYPERBOLIC LOCUS NUH

$$
\mathrm{NUH}_{\chi}^{\#}=\left\{x \in \mathrm{NUH}_{\chi}^{*}: \limsup _{n \rightarrow+\infty} q\left(f^{n}(x)\right)>0 \text { and } \limsup _{n \rightarrow-\infty} q\left(f^{n}(x)\right)>0\right\} .
$$

It is important to notice that this recurrence assumption is harmless for measures, since an analogue of Lemma 2.4 holds: if $\mu$ is an $f$-invariant probability measure supported on $\mathrm{NUH}_{\chi}^{*}$, then it is supported on $\mathrm{NUH}_{\chi}^{\#}$. This follows from the Poincaré recurrence theorem (we leave the details to the reader). The main result we want to discuss is the following.

Theorem 6.1. Let $f: M \rightarrow M$ be as above. For every $\chi>0$, there exists a TMS $(\Sigma, \sigma)$ and a Hölder continuous map $\pi: \Sigma \rightarrow M$ s.t.:

(1) $\pi \circ \sigma=f \circ \pi$.

(2) $\pi\left[\Sigma^{\#}\right]=\mathrm{NUH}_{\chi}^{\#}$.

(3) The restriction $\pi \uparrow_{\Sigma \#}: \Sigma^{\#} \rightarrow \mathrm{NUH}_{\chi}^{\#}$ is finite-to-one.

Remember the definition of the recurrent set $\Sigma^{\#}$ in the beginning of Part 2 . Theorem 6.1 does not rely on any measure, and instead provides a single symbolic model that codes all $\chi$-hyperbolic measures at the same time. Theorem 6.1 is a strengthening of Theorem 1.3 established by Sarig in Sar13. The main difference between Theorem 6.1 and Sar13, Thm. 1.3] is that Sarig's construction relies on Lyapunov regularity, and as a consequence he only obtains an inclusion of the form $\pi\left[\Sigma^{\#}\right] \supset \mathrm{NUH}_{\chi}^{\#}$. But performing the same arguments of his proof inside the nonuniformly hyperbolic loci we have defined here provides the above statement. This observation grew from the ongoing work with Buzzi and Crovisier [BCL], in which we need a more intrinsic construction to make it work for three dimensional flows. The proof of Theorem 6.1 makes essential use of the low dimension of $M$ : since the bundles $E^{s}, E^{u}$ are one-dimensional, we are able to apply arguments of bounded distortion. If $M$ has dimension larger than two, then $E^{s}, E^{u}$ can both have dimension larger than one, and there is no a priori reason for them to satisfy bounded distortion estimates. Nevertheless, building on his previous work BO18, Ben Ovadia was able to obtain a result similar to Theorem 6.1 that works in any dimension BO19, see more in Subsection 6.7. 
6.1.1. E-overlap of Pesin charts. We start establishing an analogue of Theorem 5.1. In the uniformly hyperbolic situation, the map $x \mapsto \Psi_{x}$ is continuous, hence the norm of $\Psi_{y}^{-1} \circ \Psi_{x}$ can be controlled by $d(x, y)$. For nonuniformly hyperbolic systems, the maps $x \in \mathrm{NUH}_{\chi} \mapsto e_{x}^{s}, e_{x}^{u}$ are not necessarily continuous, so even though $d(x, y) \ll 1$ we can still have $\Psi_{y}^{-1} \circ \Psi_{x}$ with large norm, if the behavior of $C(x)$ and $C(y)$ are very different. Therefore, we only allow overlaps when, in addition to taking nearby points, their matrices $C$ are close. For the definition, we allow Pesin charts to have different domains: for each $\eta \in I_{\varepsilon}$, define $\Psi_{x}^{\eta}:=\Psi_{x} \uparrow_{[-\eta, \eta]^{2}}$.

E-OVERLAP: Two Pesin charts $\Psi_{x_{1}}^{\eta_{1}}, \Psi_{x_{2}}^{\eta_{2}}$ are said to $\varepsilon$-overlap if $\frac{\eta_{1}}{\eta_{2}}=e^{ \pm \varepsilon}$ and $d\left(x_{1}, x_{2}\right)+\left\|\overline{C\left(x_{1}\right)}-\overline{C\left(x_{2}\right)}\right\|<\left(\eta_{1} \eta_{2}\right)^{4}$. When this happens, we write $\Psi_{x_{1}}^{\eta_{1}} \stackrel{\varepsilon}{\approx} \Psi_{x_{2}}^{\eta_{2}}$.

This notion was introduced in Sar13. It constitutes the first main ingredient in the proof of Theorem 6.1. The definition is strong enough to guarantee that the hyperbolicity parameters of $x_{1}$ and $x_{2}$ are almost the same, see [Sar13, Lemma 3.3] or [LM18, Prop. 3.4]. Now we are able to recover Theorem 5.1.

Theorem 6.2. The following holds for all $\varepsilon>0$ small enough. If $\Psi_{f(x)}^{\eta} \approx \Psi_{y}^{\eta^{\prime}}$, then $f_{x, y}$ is well-defined on $[-10 Q(x), 10 Q(x)]^{2}$ and can be written as $f_{x, y}\left(v_{1}, v_{2}\right)=$ $\left(A v_{1}+h_{1}\left(v_{1}, v_{2}\right), B v_{2}+h_{2}\left(v_{1}, v_{2}\right)\right)$ where:

(1) $|A|,\left|B^{-1}\right|<e^{-\chi}$, cf. Theorem 2.3 .

(2) $\left\|h_{i}\right\|_{1+\beta / 3}<\varepsilon$ for $i=1,2$.

If $\Psi_{f^{-1}(y)}^{\eta^{\prime}} \stackrel{\varepsilon}{\approx} \Psi_{x}^{\eta}$ then a similar statement holds for $f_{x, y}^{-1}$.

This is [Sar13, Proposition 3.4].

6.1.2. $\varepsilon$-double charts. Having a good notion of overlap between Pesin charts, now we want to define graph transforms. The approach will be similar to Subsection 2.7. when we defined stable and unstable graph transforms using different scales $q^{s}$ and $q^{u}$. In the referred subsection, we also gave a dynamical explanation of the recursive equations in Lemma 2.5(2) that $q^{s}, q^{u}$ satisfy. To extend this to Pesin charts, we consider two scales for each chart, one that controls the stable direction and another that controls the unstable direction. Hence we do not work with Pesin charts alone, but instead consider different objects, called double charts, and use them to define stable and unstable graph transforms. This idea, also introduced in Sar13], is the second main ingredient in the proof of Theorem 6.1.

$\varepsilon$-DOUBle CHART: An $\varepsilon$-double chart is a pair of Pesin charts $\Psi_{x}^{p^{s}, p^{u}}=\left(\Psi_{x}^{p^{s}}, \Psi_{x}^{p^{u}}\right)$ where $p^{s}, p^{u} \in I_{\varepsilon}$ with $0<p^{s}, p^{u} \leq Q(x)$.

Intuitively, just like $q^{s / u}(x)$ are choices for the sizes of local stable/unstable manifolds at $x$, the parameters $p^{s} / p^{u}$ represent candidates for the sizes of local stable/unstable manifolds of pseudo-orbits. To make sense of this, let us first define transitions between $\varepsilon$-double charts.

EDGE $v \stackrel{\varepsilon}{\rightarrow} w$ : Given $\varepsilon$-double charts $v=\Psi_{x}^{p^{s}}, p^{u}$ and $w=\Psi_{y}^{q^{s}}, q^{u}$, we draw an edge from $v$ to $w$ if the following conditions are satisfied:

(GPO1) $\Psi_{f(x)}^{q^{s} \wedge q^{u}} \approx \Psi_{y}^{\varepsilon} \Psi^{q^{s} \wedge q^{u}}$ and $\Psi_{f^{-1}(y)}^{p^{s} \wedge p^{u}} \underset{\approx}{\approx} \Psi_{x}^{p^{s} \wedge p^{u}}$.

(GPO2) $p^{s}=\min \left\{e^{\varepsilon} q^{s}, Q(x)\right\}$ and $q^{u}=\min \left\{e^{\varepsilon} p^{u}, Q(y)\right\}$. 
Condition (GPO1) allows to represent $f$ nearby $x$ by Pesin charts at $x$ and $y$, and similarly for $f^{-1}$. Condition (GPO2) is a greedy algorithm that chooses the local hyperbolicity parameters as largest as possible, and is the counterpart of Lemma 2.5 (2) for pseudo-orbits.

$\varepsilon$-GENERALIZED PSEUDO-ORBIT ( $\varepsilon-$ GPO): An $\varepsilon$-generalized pseudo-orbit is a se-

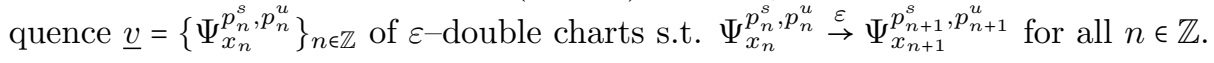

This definition is much stronger than the one given in Subsection 5.6. Observe that if $\underline{v}$ is an $\varepsilon$-gpo then by (GPO2) we have that

$$
p_{0}^{s}=\inf \left\{e^{\varepsilon n} Q\left(x_{n}\right): n \geq 0\right\} \text { and } p_{0}^{u}=\inf \left\{e^{\varepsilon n} Q\left(x_{-n}\right): n \geq 0\right\} .
$$

These equations are very similar to the definitions of $q^{s / u}$, see Subsection 2.6

6.1.3. Graph transforms. To finally define graph transforms, it remains to strengthen the notion of admissibility. Let $v=\Psi_{x}^{p^{s}}, p^{u}$ be an $\varepsilon$-double chart.

ADmissible MANIFOLDS: An $s$-admissible manifold at $v$ is a set of the form $V^{s}=$ $\Psi_{x}\left\{(t, F(t)):|t| \leq p^{s}\right\}$, where $F:\left[-p^{s}, p^{s}\right] \rightarrow \mathbb{R}$ is a $C^{1+\beta / 3}$ function s.t.:

(AM1) $|F(0)| \leq 10^{-3}\left(p^{s} \wedge p^{u}\right)$.

(AM2) $\left|F^{\prime}(0)\right| \leq \frac{1}{2}\left(p^{s} \wedge p^{u}\right)^{\beta / 3}$.

(AM3) $\left\|F^{\prime}\right\|_{C^{0}}+\operatorname{Hol}_{\beta / 3}\left(F^{\prime}\right) \leq \frac{1}{2}$ where the norms are taken in $\left[-p^{s}, p^{s}\right]$.

Similarly, a $u$-admissible manifold at $v$ is a set of the form $V^{u}=\Psi_{x}\{(G(t), t):|t| \leq$ $\left.p^{u}\right\}$ where $G:\left[-p^{u}, p^{u}\right] \rightarrow \mathbb{R}$ is a $C^{1+\beta / 3}$ function satisfying (AM1)-(AM3), where the norms are taken in $\left[-p^{u}, p^{u}\right]$.

Note that $p^{s / u}$ control the domains of the representing functions, and $p^{s} \wedge p^{u}$ controls their behaviour at 0 . Let $\mathscr{M}_{v}^{s}, \mathscr{M}_{v}^{u}$ be the space of all $s, u$-admissible manifolds at $v$, which are metric spaces with the same metrics as before. For each edge $v \stackrel{\varepsilon}{\rightarrow} w$, define the stable graph transform $\mathscr{F}_{v, w}^{s}: \mathscr{M}_{w}^{s} \rightarrow \mathscr{M}_{v}^{s}$ and the unstable graph transform $\mathscr{F}_{v, w}^{u}: \mathscr{M}_{v}^{u} \rightarrow \mathscr{M}_{w}^{u}$ as before. An analogue of Theorem 5.2 holds, and we can similarly define stable and unstable manifolds for every $\varepsilon-$ gpo $\underline{v}$.

Stable/Unstable MANifolds: The stable manifold of an $\varepsilon-$ gpo $\underline{v}=\left\{v_{n}\right\}_{n \in \mathbb{Z}}$ is the unique $s$-admissible manifold $V^{s}[\underline{v}] \in \mathscr{M}_{v_{0}}^{s}$ defined by

$$
V^{s}[\underline{v}]:=\lim _{n \rightarrow \infty}\left(\mathscr{F}_{v_{0}, v_{1}}^{s} \circ \cdots \circ \mathscr{F}_{v_{n-1}, v_{n}}^{s}\right)\left[V_{n}\right]
$$

for some (any) sequence $\left\{V_{n}\right\}_{n \geq 0}$ with $V_{n} \in \mathscr{M}_{v_{n}}^{s}$. The unstable manifold of $\underline{v}$ is the unique $u$-admissible manifold $V^{u}[\underline{v}] \in \mathscr{M}_{v_{0}}^{u}$ defined by

$$
V^{u}[\underline{v}]:=\lim _{n \rightarrow-\infty}\left(\mathscr{F}_{v_{-1}, v_{0}}^{u} \circ \cdots \circ \mathscr{F}_{v_{n}, v_{n+1}}^{u}\right)\left[V_{n}\right]
$$

for some (any) sequence $\left\{V_{n}\right\}_{n \leq 0}$ with $V_{n} \in \mathscr{M}_{v_{n}}^{u}$.

These manifolds are genuine Pesin invariant manifolds, see Sar13, Prop. 6.3]. In particular, if $y, z \in V^{s}[\underline{v}]$ then $\frac{s(y)}{s(z)}=e^{ \pm \text {const }}$, and similarly for $V^{u}[\underline{v}]$. We point out that if $F$ is the representing function of $V^{s}[\underline{v}]$ and $F_{n}$ is the representing function of $\left(\mathscr{F}_{v_{0}, v_{1}}^{s} \circ \cdots \circ \mathscr{F}_{v_{n-1}, v_{n}}^{s}\right)\left[V_{n}\right]$ for $n \geq 0$, then $\left\|F-F_{n}\right\|_{C^{1}} \rightarrow 0$ as $n \rightarrow \infty$, and the same holds for the representing function of $V^{u}[\underline{v}]$. This follows from the Arzelà-Ascoli theorem, since the $C^{1+\beta / 3}$ norm of representing functions is uniformly bounded, see e.g. part (2) in the proof of Sar13, Prop. 4.15]. We also define shadowing. 
SHADOwING: We say that $\underline{v}=\left\{\Psi_{x_{n}}^{p_{n}^{s}, p_{n}^{u}}\right\}_{n \in \mathbb{Z}}$ shadows $x$ if $f^{n}(x) \in \Psi_{x_{n}}\left(\left[-p_{n}^{s} \wedge p_{n}^{u}, p_{n}^{s} \wedge\right.\right.$ $\left.p_{n}^{u}\right]^{2}$ ) for all $n \in \mathbb{Z}$.

The Shadowing Lemma is still valid, again with $\{x\}=V^{s}[\underline{v}] \cap V^{u}[\underline{v}]$, see Sar13, Thm 4.16(1)].

6.2. Coarse graining. The third main ingredient in the proof of Theorem 6.1 consists on choosing countably many charts that shadow all orbits of interest. For uniformly hyperbolic systems, a sufficiently dense set of points is enough. For nonuniformly hyperbolic systems, the construction is more elaborate. Firstly, the definition of $\varepsilon$-overlap depends on $\|\widetilde{C(x)}-\overline{C(y)}\|$, which depends on a comparison between $s(x), u(x), \alpha(x)$ and $s(y), u(y), \alpha(y)$. Secondly, nearest neighbor conditions do not follow from control at $x$ and $y$. Fortunately, all parameters involved in the construction belong to a precompact set, so there are countable dense subsets.

Theorem 6.3. For all $\varepsilon>0$ sufficiently small, there exists a countable set $\mathscr{A}$ of $\varepsilon$-double charts with the following properties:

(1) Discreteness: For all $t>0$, the set $\left\{\Psi_{x}^{p^{s}, p^{u}} \in \mathscr{A}: p^{s}, p^{u}>t\right\}$ is finite.

(2) Sufficiency: If $x \in \mathrm{NUH}_{\chi}^{\#}$ then there is an $\varepsilon-$ gpo $\underline{v} \in \mathscr{A}^{\mathbb{Z}}$ that shadows $x$.

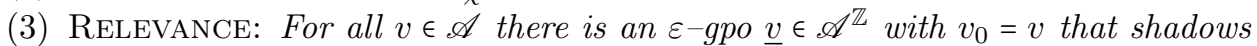
a point in $\mathrm{NUH}_{\chi}^{\#}$.

The original statement is Sar13, Thm 4.16]. The discreteness property (1) says that Pesin blocks require only finitely many $\varepsilon$-double charts, while the relevance property (3) guarantees that none of the $\varepsilon$-double charts is redundant.

Sketch of proof. Define $X:=M^{3} \times \mathrm{GL}(2, \mathbb{R})^{3} \times(0,1]$. For each $x \in \mathrm{NUH}_{\chi}^{*}$, let $\Gamma(x)=(\underline{x}, \underline{C}, \underline{Q}) \in X$ with

$$
\underline{x}=\left(f^{-1}(x), x, f(x)\right), \underline{C}=\left(C\left(f^{-1}(x)\right), C(x), C(f(x))\right), \underline{Q}=Q(x) .
$$

Let $Y=\left\{\Gamma(x): x \in \mathrm{NUH}_{\chi}^{*}\right\}$. For each triple $\underline{\ell}=\left(\ell_{-1}, \ell_{0}, \ell_{1}\right) \in \mathbb{N}_{0}^{3}$, define

$$
Y_{\underline{\ell}}:=\left\{\Gamma(x) \in Y: e^{\ell_{i}} \leq\left\|C\left(f^{i}(x)\right)^{-1}\right\|<e^{\ell_{i}+1},-1 \leq i \leq 1\right\} .
$$

Then $Y=\bigcup_{\underline{\underline{\ell}} \mathbb{N}_{0}^{3}} Y_{\underline{\underline{\ell}}}$, and each $Y_{\underline{\underline{\ell}}}$ is precompact in $X$ by definition. For each $j \geq 0$, choose a finite set $Y_{\underline{\underline{\ell}}}(j) \subset Y_{\underline{\underline{\ell}}}$ s.t. for every $\Gamma(x) \in Y_{\underline{\underline{\ell}}}$ there exists $\Gamma(y) \in Y_{\underline{\underline{\ell}}}(j)$ s.t.:

(a) $\left.\left.d\left(f^{i}(x), f^{i}(y)\right)+\| C \overline{\left(f^{i}(x)\right.}\right)-C \overline{\left(f^{i}(y)\right.}\right) \|<e^{-8(j+2)}$ for $-1 \leq i \leq 1$.

(b) $\frac{Q(x)}{Q(y)}=e^{ \pm \varepsilon / 3}$.

We define the countable set of $\varepsilon$-double charts as follows.

The alphabet $\mathscr{A}$ : Let $\mathscr{A}$ be the countable family of $\Psi_{x}^{p^{s}, p^{u}}$ s.t.:

$(\mathrm{CG} 1) \Gamma(x) \in Y_{\underline{\ell}}(j)$ for some $(\underline{\ell}, j) \in \mathbb{N}_{0}^{3} \times \mathbb{N}_{0}$.

(CG2) $0<p^{s}, p^{\underline{u}} \leq Q(x)$ and $p^{s}, p^{u} \in I_{\varepsilon}$.

(CG3) $e^{-j-2} \leq p^{s} \wedge p^{u} \leq e^{-j+2}$.

This alphabet satisfies (1) and (2) but not necessarily (3). We can easily reduce it to a sub-alphabet $\mathscr{A}^{\prime}$ satisfying (1)-(3) as follows. Call $v \in \mathscr{A}$ relevant if there is $\underline{v} \in \mathscr{A}^{\mathbb{Z}}$ with $v_{0}=v$ s.t. $\underline{v}$ shadows a point in $\mathrm{NUH}_{\chi}^{\#}$. Since $\mathrm{NUH}_{\chi}^{\#}$ is $f$-invariant, every $v_{i}$ is relevant. Then $\mathscr{A}^{\prime}=\{v \in \mathscr{A}: v$ is relevant $\}$ is discrete because $\mathscr{A}^{\prime} \subset \mathscr{A}$, it is sufficient and relevant by definition. 
Referring to the steps of Subsection 5.6.3, we have just completed Step 1. Now let $\mathscr{G}=(V, E)$ be the oriented graph with vertex set $V=\mathscr{A}$ and edge set $E=\{v \stackrel{\varepsilon}{\rightarrow} w\}$, and let $(\Sigma, \sigma)$ be the TMS generated by $\mathscr{G}$. The proof of sufficiency actually gives more: if $x \in \mathrm{NUH}_{\chi}^{\#}$ then there is a recurrent $\varepsilon-$ gpo $\underline{v} \in \Sigma^{\#}$ that shadows $x$. By the Shadowing Lemma, $\pi: \Sigma \rightarrow M$ defined by $\{\pi(\underline{v})\}:=V^{s}[\underline{v}] \cap V^{u}[\underline{v}]$ is an infinite-toone extension of $f$ s.t. $\pi\left[\Sigma^{\#}\right] \supset \mathrm{NUH}_{\chi}^{\#}$.

6.3. Improvement lemma. The fourth main ingredient in the proof of Theorem 6.1 is an important fact, that will imply the reverse inclusion $\pi\left[\Sigma^{\#}\right] \subset \mathrm{NUH}_{\chi}^{\#}$ as well as the Inverse Theorem in the next subsection. We start observing that points of $\pi\left[\Sigma^{\#}\right]$ do have stable and unstable directions. If $\underline{v}$ is an $\varepsilon$-gpo, then for $x \in V^{u}[\underline{v}]$ we can take $e_{x}^{u}$ to be (any of) the tangent vector to $V^{u}[\underline{v}]$ at $x$. This direction indeed contracts in the past and expands in the future, see Sar13, Prop. 6.3(2)] and claim (i) in the proof of $\left[\right.$ Sar13, Proposition 6.5]. Similarly, for $x \in V^{s}[\underline{v}]$ we can take (any of) the tangent vector to $V^{s}[\underline{v}]$ at $x$ to be the stable direction $e_{x}^{s}$.

Fix $\underline{v} \in \Sigma^{\#}$, and let $x=\pi(\underline{v})$. Since $\{x\}=V^{s}[\underline{v}] \cap V^{u}[\underline{v}], e_{x}^{s}, e_{x}^{u}$ are defined. If we prove that $s(x), u(x)<\infty$, then (NUH3) holds and automatically (NUH1)(NUH2) hold as well, implying that $x \in \mathrm{NUH}_{\chi}$. The proof that $s(x), u(x)<\infty$ is very delicate, since a priori there is no reason for $x$ to have a hyperbolic behaviour as good as the behaviour of the centers of the $\varepsilon$-double charts of $\underline{v}$. But the notions of $\varepsilon$-overlap and admissibility are so strong that indeed $s(x), u(\bar{x})<\infty$. The proof of this fact relies on a general philosophy that $f$ improves smoothness along the unstable direction, and $f^{-1}$ improves smoothness along the stable direction. In terms of graph transforms, the ratios of $s, u$ parameters improve. We call this an improvement lemma. The heuristics for such improvement can be explained as follows. Assume that $v \stackrel{\varepsilon}{\rightarrow} w$, where $v=\Psi_{x_{0}}^{p_{0}^{s}, p_{0}^{u}}$ and $w=\Psi_{x_{1}}^{p_{1}^{s}, p_{1}^{u}}$, let $V^{s} \in \mathscr{M}_{w}^{s}$ and $\widetilde{V}^{s}=\mathscr{F}_{v, w}^{s}\left[V^{s}\right]$, and fix a point $y \in \widetilde{V}^{s}$. In particular $f(y) \in V^{s}$, see Figure

17. Assuming that $s(y)<\infty$, we want to compare the ratios $\frac{s(f(y))}{s\left(x_{1}\right)}$ and $\frac{s(y)}{s\left(x_{0}\right)}$.
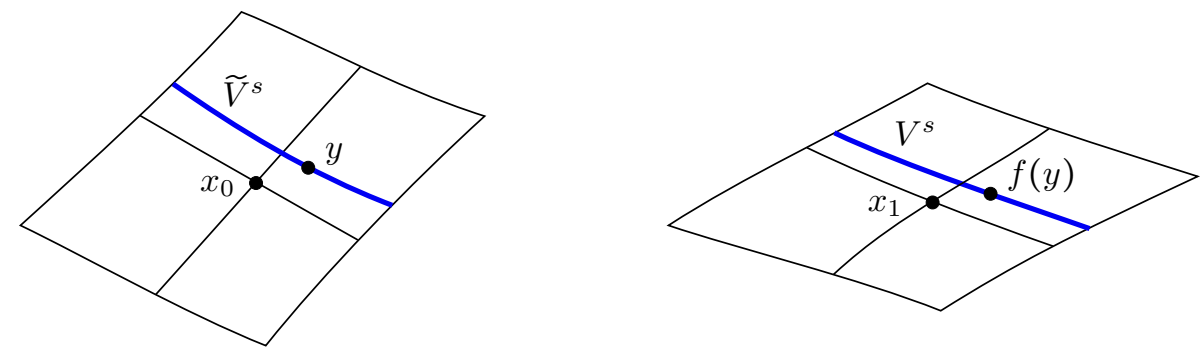

FiguRE 17. Improvement Lemma: the graph transform improves ratios.

Proceeding as in the proof of Lemma 1.1, we have $s(y)^{2}=2+C s(f(y))^{2}$, where $C=\left\|d f e_{y}^{s}\right\|^{2} e^{2 \chi}$. By the $\varepsilon$-overlap, we also have $s\left(x_{0}\right)^{2} \approx 2+C s\left(x_{1}\right)^{2}$, and so $\frac{s(y)^{2}}{s\left(x_{0}\right)^{2}} \approx \frac{2+C s(f(y))^{2}}{2+C s\left(x_{1}\right)^{2}}$. If the initial ratio is $\frac{s(f(y))^{2}}{s\left(x_{1}\right)^{2}}=K \gg 1$, then the new ratio is $\frac{s(y)^{2}}{s\left(x_{0}\right)^{2}} \approx \frac{2+K C s\left(x_{1}\right)^{2}}{2+C s\left(x_{1}\right)^{2}}<K$. The same occurs if $K \ll 1$, in which case the new ratio becomes $>K$. 
Lemma 6.4 (Improvement Lemma). The following holds for $\varepsilon>0$ small enough. For $\xi \geq \sqrt{\varepsilon}$, if $\frac{s(f(y))}{s\left(x_{1}\right)}=e^{ \pm \xi}$, then $\frac{s(y)}{s\left(x_{0}\right)}=e^{ \pm\left(\xi-Q\left(x_{0}\right)^{\beta / 4}\right)}$.

Hence the ratio improves whenever it is outside $\left[e^{-\sqrt{\varepsilon}}, e^{\sqrt{\varepsilon}}\right]$, see Sar13, Lemma 7.2] for a proof. Here is the first main consequence of this important lemma.

Corollary 6.5. $\pi\left[\Sigma^{\#}\right] \subset \mathrm{NUH}_{\chi}$, i.e. if $x=\pi(\underline{v})$ for $\underline{v} \in \Sigma^{\#}$, then $s(x), u(x)<+\infty$.

The proof can be found in Sar13, §7.1]. We summarize it as follows. Let $v_{n_{k}}=v$ for infinitely many $n_{k}>0$. Since $v=\Psi_{z}^{p^{s}, p^{u}}$ is relevant, there is $V^{s} \in \mathscr{M}_{v}^{s}$ s.t. $s(y)<\infty$ for every $y \in V^{s}$. Starting with $V_{n_{k}}=V^{s}$, pull it back through the graph transforms to get $\widetilde{V}_{k}^{s} \in \mathscr{M}_{v_{0}}^{s}$. If $k$ is large enough, then the original ratio $\frac{s(y)}{s(z)}$ passed through sufficiently many improvements $Q(z)$ so that $\frac{s\left(w_{k}\right)}{s\left(x_{0}\right)}=e^{ \pm \xi}$ for all $w_{k} \in \widetilde{V}_{k}^{s}$, for some fixed $\xi \geq \sqrt{\varepsilon}$. Since the representing functions of $\widetilde{V}_{k}^{s}$ converge to the representing function of $V^{s}[\underline{v}]$ in the $C^{1}$ norm, we conclude that $s(x)<\infty$. Similarly, we obtain that $u(x)<\infty$.

6.4. Inverse theorem. The fifth and final main ingredient in the proof of Theorem 6.1 is the Inverse Theorem. To understand its importance, recall that once we have constructed an infinite-to-one coding $\pi$, the next step is to apply a Bowen-Sinar refinement to the Markov cover $\mathscr{Z}$ induced by $\pi$. Since $\Sigma$ has countably many states, the Markov cover is countable, so that after refining the resulting partition could be uncountabl 9 . One condition that guarantees an yet countable refinement is local finiteness: $\mathscr{Z}$ is locally finite if every $Z \in \mathscr{Z}$ only intersects finitely many others $Z^{\prime} \in \mathscr{Z}$. The understanding of intersections $Z \cap Z^{\prime}$ comes from an inverse problem: if $\pi(\underline{v})=x$, how is $\underline{v}$ defined in terms of $x$ ? The next theorem (essentially) answers this question.

Theorem 6.6 (Inverse Theorem). Let $\underline{v}=\left\{\Psi_{x_{n}}^{p_{n}^{s}, p_{n}^{u}}\right\}_{n \in \mathbb{Z}} \in \Sigma^{\#}$, and let $\pi(\underline{v})=x$. Then the following holds for all $n \in \mathbb{Z}$ :

(1) Control of $x$ : $\operatorname{dist}\left(x_{n}, f^{n}(x)\right)<$ const.

(2) Control of $\alpha: \frac{\sin \alpha\left(x_{n}\right)}{\sin \alpha\left(f^{n}(x)\right)}=e^{ \pm \text {const }}$.

(3) Control OF $s, u: \frac{s\left(x_{n}\right)}{s\left(f^{n}(x)\right)}=\frac{u\left(x_{n}\right)}{u\left(f^{n}(x)\right)}=e^{ \pm \mathrm{const}}$.

(4) Control of $p^{s}, p^{u}: \frac{p_{n}^{s}}{q^{s}\left(f^{n}(x)\right)}=\frac{p_{n}^{u}}{q^{u}\left(f^{n}(x)\right)}=e^{ \pm \text {const }}$.

In particular $x \in \mathrm{NUH}_{\chi}^{\#}$, and so $\pi\left[\Sigma^{\#}\right]=\mathrm{NUH}_{\chi}^{\#}$.

The above theorem is not the original reference [Sar13, §6], since it did not make use of $q, q^{s}, q^{u}$. Instead, the original statement considered two $\varepsilon$-gpo's $\underline{v}, \underline{w} \in \Sigma^{\#}$ and compared their parameters directly. The constant appearing in the theorem is of the order of $\sqrt[3]{\varepsilon}$. The assumption $\underline{v} \in \Sigma^{\#}$ is essential to guarantee parts (3) and (4), since the proof uses that the trajectories visit a Pesin block infinitely often. Theorem 6.6 states that each coordinate of $\underline{v}$ is uniquely defined "up to bounded error". Below we explain how to get the estimates for $n=0$.

Control of $x$ And $\alpha$. Let $F, G$ be the representing functions of $V^{s}, V^{u}$. Since $F(0), G(0) \approx 0$, the graphs of $F, G$ intersect close to the origin $(0,0)$. Applying $\Psi_{x_{0}}$, we conclude that $d\left(x_{0}, x\right) \ll 1$. To control the angle, we use that $\left\|F^{\prime}\right\|_{C^{0}},\left\|G^{\prime}\right\|_{C^{0}} \ll 1$ and so the graphs of $F, G$ intersect almost perpendicularly. Applying $\Psi_{x_{0}}$, we get that $\alpha\left(x_{0}\right) \approx \alpha(x)$, see Figure 18 .

\footnotetext{
${ }^{9}$ For example, the refinement of the dyadic intervals in $[0,1]$ is the point partition.
} 


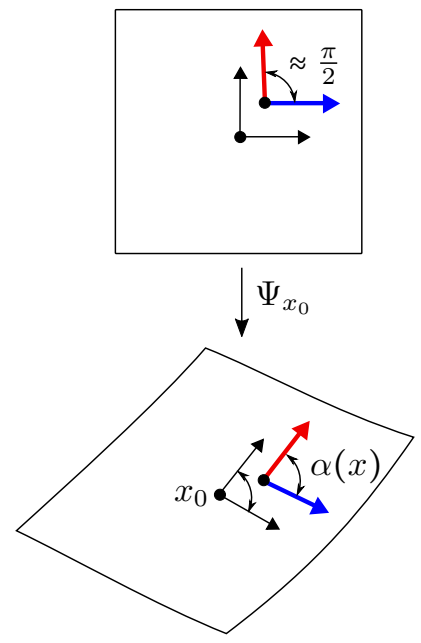

Figure 18. Control of $x$ and $\alpha$.

Control of $s, u$. The proof is identical to the proof of Corollary 6.5 .

Observe that parts (2)-(3) imply that $\frac{Q\left(x_{n}\right)}{Q\left(f^{n}(x)\right)}=e^{ \pm \text {const }}$ for all $n \in \mathbb{Z}$.

Control of $p^{s}, p^{u}$. We prove the first estimate $\frac{p_{0}^{s}}{q^{s}(x)}=e^{ \pm \text {const }}$ (the second is analogous). The idea is to observe that $p_{0}^{s}$ and $q^{s}(x)$ are defined as infima of comparable sequences. Indeed, by definition we have

$$
q^{s}(x)=\inf \left\{e^{\varepsilon n} Q\left(f^{n}(x)\right): n \geq 0\right\}
$$

and by (GPO2) we have

$$
p_{0}^{s}=\inf \left\{e^{\varepsilon n} Q\left(x_{n}\right): n \geq 0\right\} .
$$

Since $\frac{Q\left(x_{n}\right)}{Q\left(f^{n}(x)\right)}=e^{ \pm \text {const }}$ for all $n \geq 0$, it follows that $\frac{p_{0}^{s}}{q^{s}(x)}=e^{ \pm \text {const }}$.

The above argument differs from the original one, and does not require any maximality assumption on $q^{s}$. Indeed, it comes for free from the recurrence of $\underline{v}$.

6.5. Bowen-Sinar̆ refinement. Local finiteness allows us to refine the Markov cover of $\mathrm{NUH}_{\chi}^{\#}$ and obtain a countable Markov partition. As mentioned in Subsection 5.1 the notion of Markov cover/partition for nonuniformly hyperbolic systems will be weaker than the one for uniformly hyperbolic systems, since we are not able to control the topology of the rectangles. Indeed, due to Theorem 6.6. the Markov cover is the projection of the canonical partition of $\Sigma^{\#}$ (instead of $\Sigma$ ) into cylinders at the zeroth position, hence rectangles will not have the regularity property. This loss of topological control compels us to perform the refinement in a more abstract way, and then check that the resulting partition still generates a finite-toone symbolic extension. The reader who is already comfortable with the uniformly hyperbolic situation will not see much difficulty in the adaptations.

The Markov cover $\mathscr{Z}:$ Let $\mathscr{Z}:=\{Z(v): v \in \mathscr{A}\}$, where

$$
Z(v):=\left\{\pi(\underline{v}): \underline{v} \in \Sigma^{\#} \text { and } v_{0}=v\right\} .
$$


Restricted to $\Sigma^{\#}$, cylinders are neither closed nor empty, so the same occurs to $Z(v)$. Nevertheless, $s / u$-fibres are still well-defined in $\mathscr{Z}$.

Proposition 6.7. The following are true.

(1) COVERING PROPERTy: $\mathscr{Z}$ is a cover of $\mathrm{NUH}_{\chi}^{\#}$.

(2) Product structure: For every $Z \in \mathscr{Z}$ and every $x, y \in Z$, the intersection $W^{s}(x, Z) \cap W^{u}(y, Z)$ consists of a single point, and this point belongs to $Z$.

(3) Symbolic Markov Property: If $x=\pi(\underline{v})$ with $\underline{v}=\left\{v_{n}\right\}_{n \in \mathbb{Z}} \in \Sigma^{\#}$, then

$f\left(W^{s}\left(x, Z\left(v_{0}\right)\right)\right) \subset W^{s}\left(f(x), Z\left(v_{1}\right)\right)$ and $f^{-1}\left(W^{u}\left(f(x), Z\left(v_{1}\right)\right)\right) \subset W^{u}\left(x, Z\left(v_{0}\right)\right)$.

(4) Local Finiteness: For every $Z \in \mathscr{Z}$, the set $\left\{Z^{\prime} \in \mathscr{Z}: Z \cap Z^{\prime} \neq \varnothing\right\}$ is finite.

Parts (1)-(3) are analogues of Proposition 5.4. The novelty is part (4), which follows from Theorem 6.3(1) and Theorem 6.6(3): if $v=\Psi_{x}^{p^{s}}, p^{u}$ and $w=\Psi_{y}^{q^{s}}, q^{u}$ satisfy $Z(v) \cap Z(w) \neq \varnothing$ then $\frac{p^{s}}{q^{s}}=\frac{p^{u}}{q^{u}}=e^{ \pm \text {const }}$, hence

$$
\#\{Z(w) \in \mathscr{Z}: Z(v) \cap Z(w) \neq \varnothing\} \leq \#\left\{w \in \mathscr{A}: q^{s}, q^{u} \geq e^{-\mathrm{const}}\left(p^{s} \wedge p^{u}\right)\right\}<\infty .
$$

Inside each $Z \in \mathscr{Z}$, define the Smale bracket $[\cdot, \cdot]_{Z}$ as before. Lemma 5.5 remains valid, see $[$ Sar13, Lemmas $10.7,10.8,10.10]$. We now refine $\mathscr{Z}$. For $Z, Z^{\prime} \in \mathscr{Z}$ s.t. $Z \cap Z^{\prime} \neq \varnothing$, let $\mathscr{E}_{Z Z^{\prime}}=$ cover of $Z$ by rectangles:

$$
\begin{aligned}
& E_{Z, Z^{\prime}}^{s u}=\left\{x \in Z: W^{s}(x, Z) \cap Z^{\prime} \neq \varnothing, W^{u}(x, Z) \cap Z^{\prime} \neq \varnothing\right\} \\
& E_{Z, Z^{\prime}}^{s \varnothing}=\left\{x \in Z: W^{s}(x, Z) \cap Z^{\prime} \neq \varnothing, W^{u}(x, Z) \cap Z^{\prime}=\varnothing\right\} \\
& E_{Z, Z^{\prime}}^{\varnothing u}=\left\{x \in Z: W^{s}(x, Z) \cap Z^{\prime}=\varnothing, W^{u}(x, Z) \cap Z^{\prime} \neq \varnothing\right\} \\
& E_{Z, Z^{\prime}}^{\varnothing \varnothing}=\left\{x \in Z: W^{s}(x, Z) \cap Z^{\prime}=\varnothing, W^{u}(x, Z) \cap Z^{\prime}=\varnothing\right\} .
\end{aligned}
$$

The above definition is simpler than the one for uniformly hyperbolic systems, since we do not take relative interiors nor closures. Let $\mathscr{R}$ be the partition that refines all of $\mathscr{E}_{Z Z^{\prime}}$. Again due to Theorem 6.3(1) and Theorem 6.6(3), $\mathscr{R}$ and $\mathscr{Z}$ satisfy two additional local finiteness properties:

- For all $R \in \mathscr{R}$, the set $\{Z \in \mathscr{Z}: Z \supset R\}$ is finite.

○ For all $Z \in \mathscr{Z}$, the set $\{R \in \mathscr{R}: R \subset Z\}$ is finite.

Inside each $R \in \mathscr{R}$, the Smale brackets $[\cdot, \cdot]_{Z}$ do not depend on $Z$, hence we can define $[\cdot, \cdot]$ on $R$.

Lemma 6.8. $\mathscr{R}$ is a Markov partition:

(1) Product structure: If $x, y \in R \in \mathscr{R}$ then $[x, y] \in R$.

(2) Markov property: if $R, S \in \mathscr{R}$ and if $x \in R, f(x) \in S$ then

$$
f\left(W^{s}(x, R)\right) \subset W^{s}(f(x), S) \text { and } f^{-1}\left(W^{u}(f(x), S)\right) \subset W^{u}(x, R) .
$$

Let $\widehat{\mathscr{G}}=(\widehat{V}, \widehat{E})$ be the graph with $\widehat{V}=\mathscr{R}$ and $\widehat{E}=\{R \rightarrow S: f(R) \cap S \neq \varnothing\}$ (compare this definition with the one given in Step 3 of Subsection 5.6.3). Let $(\widehat{\Sigma}, \widehat{\sigma})$ be the TMS defined by $\widehat{\mathscr{G}}$, and define $\widehat{\pi}: \Sigma \rightarrow M$ by

$$
\{\widehat{\pi}(\underline{R})\}:=\bigcap_{n \geq 0} \overline{f^{n}\left(R_{-n}\right) \cap \cdots \cap f^{-n}\left(R_{n}\right)} .
$$

In comparison to the previous constructions, we take closures because the $R_{n}$ 's are not necessarily closed. A priori, the image of $\widehat{\pi}$ could be much bigger than the image of $\pi$. Fortunately, this is not the case: for each $\underline{R} \in \widehat{\Sigma}^{\#}$, there is an 
$\varepsilon-$ gpo $\underline{v} \in \Sigma^{\#}$ s.t. $\widehat{\pi}(\underline{R})=\pi(\underline{v})$, see the proof of $\operatorname{Sar13}$, Thm 12.5]. Therefore $\widehat{\pi}\left[\widehat{\Sigma}^{\#}\right]=\pi\left[\Sigma^{\#}\right]=\mathrm{NUH}_{\chi}^{\#}$.

We point out that $\widehat{\pi}$ is compatible with the Smale brackets in $\Sigma$ and $\mathscr{R}$. More specifically, for $\underline{R}=\left\{R_{n}\right\}_{n \in \mathbb{Z}}, \underline{S}=\left\{S_{n}\right\}_{n \in \mathbb{Z}} \in \Sigma$ with $R_{0}=S_{0}$, let $\underline{U}=[\underline{R}, \underline{S}]$ where $\underline{U}=\left\{U_{n}\right\}_{n \in \mathbb{Z}}$ is defined by

$$
U_{n}=\left\{\begin{array}{cl}
R_{n} & , \text { if } n \geq 0 \\
S_{n} & , \text { if } n \leq 0
\end{array}\right.
$$

Then $\widehat{\pi}([\underline{R}, \underline{S}])=[\widehat{\pi}(\underline{R}), \widehat{\pi}(\underline{S})]$. This is BPVL19, Lemma 4.4], and it is used to study the simplicity of generic fiber-bunched cocycles over nonuniformly hyperbolic diffeomorphisms.

6.6. Affiliation and Bowen relation. We investigate how $\widehat{\pi}: \widehat{\Sigma}^{\#} \rightarrow \mathrm{NUH}_{\chi}^{\#}$ loses injectivity. In the uniformly hyperbolic situation, we saw in Subsection 5.6.4 that this is characterized by the Bowen relation. Sarig was able to obtain a similar characterization [Sar13], which was further explored by Boyle and Buzzi BB17.

Affiliation: Two rectangles $R, S \in \mathscr{R}$ are called affiliated if there exist $Z, Z^{\prime} \in \mathscr{Z}$ s.t. $Z \supset R, Z^{\prime} \supset S$ and $Z \cap Z^{\prime} \neq \varnothing$. If this occurs, we write $R \sim S$. See Figure 19 .

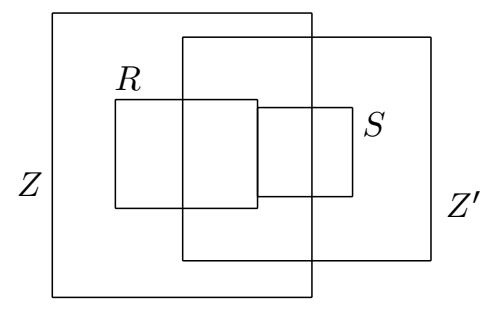

Figure 19. The affiliation property $R \sim S$ : it might occur even when $R \cap S=\varnothing$.

Affiliation is more complicated than mere nonempty intersection, and it arises from the need of taking closures in the definition of $\widehat{\pi}$. If $R \sim S$ as above, then Lemma 5.5 implies that we can take Smale brackets between points of $Z$ and $Z^{\prime}$.

Bowen Relation: We say that $\underline{R} \approx \underline{S}$ iff $R_{n} \sim S_{n}$ for all $n \in \mathbb{Z}$.

The following result was implicit in [Sar13, as explained in [BB17, §8.3].

Lemma 6.9. If $\underline{R}, \underline{S} \in \widehat{\Sigma}^{\#}$, then $\widehat{\pi}(\underline{R})=\widehat{\pi}(\underline{S})$ iff $\underline{R} \approx \underline{S}$.

Now we apply the diamond argument, as in Subsection 5.6.4, by choosing $n<m$ s.t. the rectangle configuration $\left(R, Z, S, Z^{\prime}\right)$ of Figure 19 is that same at positions $n$ and $m$. Introduce

$$
N(R):=\#\left\{\left(S, Z^{\prime}\right) \in \mathscr{R} \times \mathscr{Z}: R \sim S \text { and } Z^{\prime} \supset S\right\} .
$$

The local finiteness of $\mathscr{R}$ and $\mathscr{Z}$ imply that $N(R)<\infty$ for all $R \in \mathscr{R}$. For fixed $R, S \in \mathscr{R}$ and $n<m$, the pigeonhole principle implies that if there are more than $N(R) N(S)$ paths $R_{n} \rightarrow \cdots \rightarrow R_{m}$ s.t. $R_{n} \sim R$ and $R_{m} \sim S$, then two of them have the same rectangle configuration at iterates $n$ and $m$, as expressed below. 
Theorem 6.10. Let $\underline{R} \in \widehat{\Sigma}^{\#}$, with $R_{n}=R$ for infinitely many $n<0$ and $R_{m}=S$ for infinitely many $m>0$, and let $x=\widehat{\pi}(\underline{R})$. Then $\#\left[\widehat{\pi}^{-1}(x) \cap \widehat{\Sigma}^{\#}\right] \leq N(R) N(S)$. In particular, the restriction $\widehat{\pi} \Gamma_{\widehat{\Sigma} \#}: \widehat{\Sigma}^{\#} \rightarrow \mathrm{NUH}_{\chi}^{\#}$ is finite-to-one.

The original proof [Sar13, Thm 12.8] has a small error that was corrected in LS19. This concludes the proof of Theorem 6.1.

6.7. Higher dimensions. Recently, building on his previous work BO18, Ben Ovadia obtained a higher dimensional version of Theorem 6.1 BO19]. Regarding the five main ingredients, the first three can be adapted to higher dimensions, with many extra technical difficulties, see BO18, Sections 2 and 3]. But the improvement lemma and inverse theorem require different approaches. Indeed, each $x=\pi(\underline{v})$ has many parameters $S(x, v)$ and $U(x, w)$, and in principle it is not clear to which reference parameters they should be compared with. Let us explain how to do the estimates for $S(x, v)$. Let $V^{s}[\underline{v}]=\Psi_{x_{0}}\left\{(t, F(t)): t \in\left[-p_{0}^{s}, p_{0}^{s}\right]^{d_{s}}\right\}$ be the stable manifold of $\underline{v}=\left\{\Psi_{x_{0}}^{p_{n}^{s}, p_{n}^{u}}\right\}_{n \in \mathbb{Z}} \in \Sigma^{\#}$. Let $x \in V^{s}[\underline{v}]$, with $x=\Psi_{x_{0}}(\bar{x})=\Psi_{x_{0}}(\bar{t}, F(\bar{t}))$. The candidate for stable subspace at $x$ is $\widetilde{E}_{x}^{s}=\left(d \Psi_{x_{0}}\right)_{\bar{x}}\left[H_{\bar{x}}^{s}\right]$, where $H_{\bar{x}}^{s}$ is the subspace tangent to $\left\{(t, F(t)): t \in\left[-p_{0}^{s}, p_{0}^{s}\right]^{d_{s}}\right\}$ at $\bar{x}$. Observing that the stable subspace at $x_{0}$ is $\left(d \Psi_{x_{0}}\right)_{0}\left[\mathbb{R}^{d_{s}} \times\{0\}\right]$, consider a linear transformation $H_{\bar{x}}^{s} \rightarrow \mathbb{R}^{d_{s}} \times$ $\{0\}$ given by the derivative of the projection $(t, F(t)) \mapsto(t, 0)$ at $\bar{x}=(\bar{t}, F(\bar{t}))$. Applying $d \Psi_{x_{0}}$, we obtain a linear transformation $\Xi_{x}: \widetilde{E}_{x}^{s} \rightarrow E_{x_{0}}^{s}$. Since the graph of $F$ is almost horizontal, $\Xi_{x}$ is almost an isometry. The improvement lemma can be stated as follows: if $v \in \widetilde{E}_{f(y)}^{s}$ satisfies $\frac{S(f(y), v)}{S\left(x_{1}, \Xi_{f(y)}\right)}=\exp [ \pm \xi]$ for some $\xi \geq \sqrt{\varepsilon}$, then $\frac{S(y, w)}{S\left(x_{0}, \Xi_{y} w\right)}=\exp \left[ \pm\left(\xi-\frac{1}{6} Q\left(x_{0}\right)^{\beta / 6}\right)\right]$ for $w=d f_{f(y)}^{-1} v$, see BO18, Lemma 4.6]. This improvement lemma implies two properties:

$\circ \pi\left[\Sigma^{\#}\right] \subset \mathrm{NUH}_{\chi}$, see Claim 1 in the proof of BO18, Lemma 4.7].

$\circ$ Inverse theorem, see [BO18, Section 4].

6.8. Uniform hyperbolicity versus nonuniform hyperbolicity. Below we summarize the main differences between the constructions of symbolic models for uniformly hyperbolic and nonuniformly hyperbolic systems. 


\begin{tabular}{|c|c|c|}
\hline & UNIFORMLY HYPERBOLIC & NON-UNIFORMLY HYPERBOLIC \\
\hline Coding & All points & $\mathrm{NUH}_{\chi}^{\#}$ \\
\hline Chart & Lyapunov chart: uniform size & Pesin chart: size $Q$ with $\frac{1}{n} \log Q\left(f^{n}(x)\right) \rightarrow 0$ \\
\hline Vertices & Finite number of Lyapunov charts & Countable number of $\varepsilon$-double charts \\
\hline Edges & $\Psi_{x} \rightarrow \Psi_{y}: f(x) \approx y$ and $f^{-1}(y) \approx x$ & $\Psi_{x}^{p^{s}, p^{u}} \stackrel{\varepsilon}{\rightarrow} \Psi_{y}^{q^{s}}, q^{u}:(\mathrm{GPO} 1)$ and (GPO2) \\
\hline Rep. function & $F \in C^{1}$ s.t. $F(0) \approx 0$ and $\left\|F^{\prime}\right\|_{C^{0}} \approx 0$ & $F \in C^{1+\beta / 3}$ with (AM1)-(AM3) \\
\hline$\pi: \Sigma \rightarrow M$ & $\{\pi(\underline{v})\}=V^{s}[\underline{v}] \cap V^{u}[\underline{v}]$ & Same \\
\hline Cover $\mathscr{Z}$ & $Z(v)=\left\{\pi(\underline{v}): v_{0}=v\right\}$ closed sets & $Z(v)=\left\{\pi(\underline{v}): \underline{v} \in \Sigma^{\#}, v_{0}=v\right\}$ \\
\hline Refinement & Relative interiors and closures & Set-theoretical refinement \\
\hline Partition $\mathscr{R}$ & Markov with regular rectangles & Markov w/o control of relative interiors \\
\hline $\operatorname{Graph}(\widehat{V}, \widehat{E})$ & $\widehat{V}=\mathscr{R}, \widehat{E}=\left\{R \rightarrow S: f\left(R^{*}\right) \cap S^{*} \neq \varnothing\right\}$ & $\widehat{V}=\mathscr{R}, \widehat{E}=\{R \rightarrow S: f(R) \cap S \neq \varnothing\}$ \\
\hline$\widehat{\pi}: \widehat{\Sigma} \rightarrow M$ & $\{\widehat{\pi}(\underline{R})\}=\cap_{n \geq 0} f^{n}\left(R_{-n}\right) \cap \cdots \cap f^{-n}\left(R_{n}\right)$ & $\{\widehat{\pi}(\underline{R})\}=\cap_{n \geq 0} \overline{f^{n}\left(R_{-n}\right) \cap \cdots \cap f^{-n}\left(R_{n}\right)}$ \\
\hline Finite-to-one & $\# \widehat{\pi}^{-1}(x) \leq(\# \mathscr{R})^{2}, \forall x \in M$ & $\#\left[\widehat{\pi}^{-1}(x) \cap \widehat{\Sigma}^{\#}\right]<\infty, \forall x \in M$ \\
\hline
\end{tabular}

6.9. Surface maps with discontinuities. Now we explain the constructions in the contexts of Sections 3, 4. Let us start with surface maps with discontinuities and bounded derivative. As in the previous section, take $I_{\varepsilon}:=\left\{e^{-\frac{1}{3} \varepsilon n}: n \geq 0\right\}$ and truncate $Q(x)$ to $I_{\varepsilon}$, then consider $\varepsilon$-overlap and $\varepsilon$-gpo as in Subection 6.1. To prove Theorem 6.3, we need to control more parameters: for $\underline{\ell}=\left(\ell_{-1}, \ell_{0}, \ell_{1}\right)$ and $\underline{k}=\left(k_{-1}, k_{0}, k_{1}\right)$, define

$$
Y_{\underline{\ell}, \underline{k}}:=\left\{\Gamma(x) \in Y: \begin{array}{ll}
e^{\ell_{i}} \leq\left\|C\left(f^{i}(x)\right)^{-1}\right\|<e^{\ell_{i}+1}, & -1 \leq i \leq 1 \\
e^{-k_{i}-1} \leq d\left(f^{i}(x), \mathscr{D}\right)<e^{-k_{i}}, & -1 \leq i \leq 1
\end{array}\right\} .
$$

Using these precompact sets, proceed as in the proof of Theorem 6.3. Theorem 6.6 works without modification, as well as Theorem 6.1.

As mentioned in Section 3, the prototypical examples for surface maps with discontinuities and bounded derivative are Poincaré return maps of three dimensional flows with positive speed. Let $N$ be a three dimensional closed Riemannian manifold, let $X$ be a $C^{1+\beta}$ vector field on $N$ s.t. $X(p) \neq 0$ for all $p \in N$, and let $\varphi=\left\{\varphi^{t}\right\}_{t \in \mathbb{R}}$ be the flow generated by $X$. In Section 3 , we constructed a global Poincaré section $M$, equal to the finite union of transverse discs, s.t. the return time function $\mathfrak{t}: M \rightarrow(0, \infty)$ is bounded away from zero and infinity. Then the Poincaré return map $f: M \rightarrow M$ has discontinuities and bounded derivative.

Now we relate the hyperbolic properties of $\varphi$ and $f$. Let $\widetilde{\chi}>0$. It is possible to define a nonuniformly hyperbolic locus $\operatorname{NUH}_{\widetilde{\chi}}(\varphi)$ for $\varphi$, similar to the definition in page 19. This is part of an ongoing project with Buzzi and Crovisier [BCL]. Let $\mathrm{NUH}_{\chi}$ be the nonuniformly hyperbolic locus of $f$. If $x \in M \cap \mathrm{NUH}_{\widetilde{\chi}}(\varphi)$, then 
$x$ satisfies (NUH1)-(NUH3) for $\chi:=\tilde{\chi} \inf (\mathfrak{t})$. Indeed, the flow trajectory of $x$ spends at least time $\inf (\mathfrak{t})$ between visits to $M$, see [S19, Lemma 2.6]. To prove that $x \in \mathrm{NUH}_{\chi}$, it remains to check (NUH4). Here we encounter a problem: the section $M$ could be chosen in such a bad way that every trajectory of $f$ converges exponentially fast to $\mathscr{D}$, and so (NUH4) never holds. To bypass this difficulty, we need to choose $M$ carefully so that most $\varphi$-trajectories define $f$-trajectories satisfying (NUH4). Unfortunately, we do not know how to construct such section. What we know, and this is done in LS19, is to construct one section for each fixed measure (more generally, for a countable set of fixed measures). Fortunately, this is enough for many applications.

Let $\mu$ be a $\widetilde{\chi}$-hyperbolic probability measure for $\varphi$, and let $\nu$ be its projection to $M$, which is $\chi$-hyperbolic for $f$. The goal is to choose $M$ so that $\nu$ is $f$-adapted. Consider a 1-parameter family of global Poincaré sections $\left\{M_{r}\right\}$, by changing the radii of each disc of $M$. More specifically, let $M_{r}=D_{r}\left(p_{1}\right) \cup \cdots \cup D_{r}\left(p_{\ell}\right), r \in[a, b]$, s.t. each $M_{r}$ is a global Poincaré section for $\varphi$ and $\mathfrak{t}_{r}: M_{r} \rightarrow(0, \infty)$ is uniformly bounded away from zero and infinity. Let $f_{r}: M_{r} \rightarrow M_{r}$ be the Poincaré return map, and let $\nu_{r}$ be the projection of $\mu$ to $M_{r}$. The next result is LS19, Thm 2.8].

Theorem 6.11. For Lebesgue-a.e. $r \in[a, b]$, the measure $\nu_{r}$ is $f_{r}$-adapted.

Proof. Let $\mathscr{D}_{r}$ be the discontinuity set of $f_{r}$. It is enough to show that

$$
\nu_{r}\left\{x \in M_{r}: \liminf _{|n| \rightarrow \infty} \frac{1}{|n|} \log d\left(f_{r}^{n}(x), \mathscr{D}_{r}\right)<0\right\}=0 \text { for a.e. } r \in[a, b] .
$$

For $\alpha>0$, let

$$
A_{\alpha}(r):=\left\{x \in M_{b}: \exists \text { infinitely many } n \in \mathbb{Z} \text { s.t. } \frac{1}{|n|} \log d\left(f_{b}^{n}(x), \mathscr{D}_{r}\right)<-\alpha\right\} .
$$

It is enough to prove that $\nu_{b}\left[A_{\alpha}(r)\right]=0$ for Lebesgue-a.e. $r \in[a, b]$. Let $I_{\alpha}(x):=$ $\left\{a \leq r \leq b: x \in A_{\alpha}(r)\right\}$. Since $1_{A_{\alpha}(r)}(x)=1_{I_{\alpha}(x)}(r)$, by Fubini's Theorem we have $\int_{a}^{b} \nu_{b}\left[A_{\alpha}(r)\right] d r=\int_{M_{b}} \operatorname{Leb}\left[I_{\alpha}(x)\right] d \nu_{b}(x)$, so it is enough to prove that

$$
\operatorname{Leb}\left[I_{\alpha}(x)\right]=0 \text { for all } x \in M_{b} \text {. }
$$

The set $I_{\alpha}(x)$ is contained in the limsup of intervals $\left\{I_{n}\right\}_{n \in \mathbb{Z}}$ with $\left|I_{n}\right| \approx e^{-\alpha|n|}$. Since $\sum_{n \in \mathbb{Z}} e^{-\alpha|n|}<\infty$, by the Borel-Cantelli lemma we get that Leb $\left[I_{\alpha}(x)\right]=0$.

Combining Theorems 6.1 and 6.11, we obtain the following result, proved in LS19] (see page 32 for the definition of TMF).

Theorem 6.12. Let $\varphi: N \rightarrow N$ be as above. For each $\chi$-hyperbolic measure $\mu$, there is a TMF $\left(\Sigma_{r}, \sigma_{r}\right)$ and $\pi_{r}: \Sigma_{r} \rightarrow N$ Hölder continuous s.t.:

(1) $\pi_{r} \circ \sigma_{r}^{t}=\varphi^{t} \circ \pi_{r}$ for all $t \in \mathbb{R}$

(2) $\pi_{r}\left[\Sigma_{r}^{\#}\right]$ has full $\mu$-measure.

(3) $\pi_{r}$ is finite-to-one on $\pi_{r}\left[\Sigma_{r}^{\#}\right]$.

Now consider surface maps with discontinuities and unbounded derivative. In some sense, the definition of $Q(x)$ given in page 30 allows to concentrate the difficulty in this single parameter, but the statements need to be reproved using this new definition. Let us see how to get Theorem 6.3. Remember from Subsection 4.1 that $f$ is well-behaved inside each ball $D_{x}=B(x, \mathfrak{r}(x))$. For $t>0$, let $M_{t}=\{x \in M: d(x, \mathscr{D}) \geq t\}$. Since $M$ has finite diameter (we are even assuming it is 
smaller than one), each $M_{t}$ is precompact. Fix a countable open cover $\mathscr{P}=\left\{D_{i}\right\}_{i \in \mathbb{N}_{0}}$ of $M \backslash \mathscr{D}$ s.t.:

○ $D_{i}:=D_{z_{i}}=B\left(z_{i}, \mathfrak{r}\left(z_{i}\right)\right)$ for some $z_{i} \in M$.

○ For every $t>0,\left\{D \in \mathscr{P}: D \cap M_{t} \neq \varnothing\right\}$ is finite.

For $\underline{\ell}=\left(\ell_{-1}, \ell_{0}, \ell_{1}\right), \underline{k}=\left(k_{-1}, k_{0}, k_{1}\right), \underline{a}=\left(a_{-1}, a_{0}, a_{1}\right)$, define

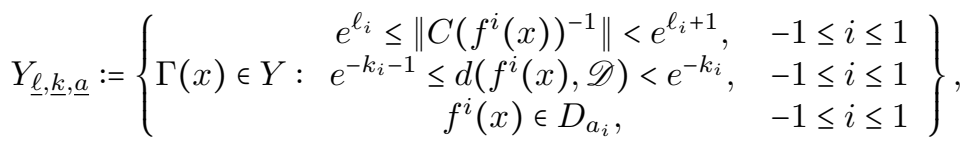

then proceed as in the proof of Theorem 6.3 .

Another feature that requires a better control is bounded distortion inside each $V^{s / u}[\underline{v}]$. This is proved in LM18, Proposition 6.2]. In summary, under finer analysis, it is possible to prove all that is needed to obtain Theorem 6.1. This is LM18, Theorem 1.3], and establishes problem \#17 in Bowen's notebook [Bow17. The proof actually works under greater generality that covers not only billiard maps but also some situations where the derivative of $M$ and the behavior of $\exp _{x}$ is more complicated, for instance when $R, \nabla R, \nabla^{2} R, \nabla^{3} R$ grow at most polynomially fast with respect to the distance to $\mathscr{D}$, e.g. when $M$ is a moduli space of curves equipped with the Weil-Petersson metric, see BMW12.

\section{Part 3. Applications}

There are two canonical applications of Markov partitions:

- Estimating the number of closed orbits.

- Establishing ergodic properties of equilibrium measures.

Indeed, the Markov partition generates a finite-to-one extension of $\mathrm{NUH}_{\chi}^{\#}$, and it is possible to lift measures without increasing their entropy.

Let $X$ be a set, $\mathscr{A}$ a sigma-algebra, and $T: X \rightarrow X$ a measurable $\mathbb{Z}$ or $\mathbb{R}$-action. Given a $T$-invariant probability measure $\mu$, let $h_{\mu}(T)$ denote its Kolmogorov-Sina $\breve{~}$ entropy. Given two such systems $(X, \mathscr{A}, T)$ and $(Y, \mathscr{B}, S)$, let $\pi:(X, \mathscr{A}) \rightarrow(Y, \mathscr{B})$ be a surjective measurable extension map, i.e. $\pi \circ T=S \circ \pi$. Assume that $\pi^{-1}(y)$ is finite for all $y \in Y$.

Projection of measure: If $\widehat{\mu}$ is a $T$-invariant probability measure on $(X, \mathscr{A})$, then its projection $\mu=\widehat{\mu} \circ \pi^{-1}$ is an $S$-invariant probability measure on $(Y, \mathscr{B})$ s.t. $h_{\mu}(S)=h_{\widehat{\mu}}(T)$.

Indeed, by the Abramov-Rokhlin formula, $h_{\widehat{\mu}}(T)-h_{\mu}(S)$ is equal to the average entropy on the fibers. Since each of them is finite, they do no carry entropy. See ELW11 for a proof of the Abramov-Rokhlin formula. In general, it is much harder to lift measures without increasing the entropy. In our setting, we can do this.

LIFT OF MEASURE: If $\mu$ is an $S$-invariant probability measure on $(Y, \mathscr{B})$, then

$$
\widehat{\mu}=\int_{Y} \frac{1}{\left|\pi^{-1}(y)\right|}\left(\sum_{x \in \pi^{-1}(y)} \delta_{x}\right) d \mu(y)
$$

is a $T$-invariant probability measure on $(X, \mathscr{A})$ s.t. $h_{\mu}(S)=h_{\widehat{\mu}}(T)$.

Firstly, one has to check that $\widehat{\mu}$ is well-defined, see e.g. Sar13, Prop. 13.2]. The preservation of entropy follows again from the Abramov-Rokhlin formula. In 
particular, if the variational principle holds in $(X, \mathscr{A}, T)$ and $(Y, \mathscr{B}, S)$, then their topological entropies coincide.

The above conclusions also hold if there are sets $X^{\#} \subset X$ and $Y^{\#} \subset Y$ s.t.:

- The restriction $\pi \uparrow_{X \#}: X^{\#} \rightarrow Y^{\#}$ is finite-to-one.

- Every $T$-invariant probability measure is supported on $X^{\#}$, and every $S$-invariant probability measure is supported on $Y^{\#}$.

It is in this way that invariant measures for nouniformly hyperbolic systems relate with invariant measures for symbolic spaces. In the next two section we explain some of the applications.

\section{Estimates ON THE NUMBeR OF CLOSED ORBITS}

Given two sequences $\left\{a_{n}\right\}_{n \geq 1},\left\{b_{n}\right\}_{n \geq 1}$, let us write $a_{n} \asymp b_{n}$ if there are constants $C, n_{0}>1$ s.t. $C^{-1} \leq \frac{a_{n}}{b_{n}} \leq C$ for all $n \geq n_{0}$, and $a_{n} \sim b_{n}$ if $\lim _{n \rightarrow \infty} \frac{a_{n}}{b_{n}}=1$. Assume that $f: M \rightarrow M$ is a transitive uniformly hyperbolic diffeomorphism, and let $(\Sigma, \sigma, \pi)$ be a symbolic model. Given $n \geq 1$, let $\operatorname{Per}_{n}(f), \operatorname{Per}_{n}(\sigma)$ denote the number of periodic orbits of period $n$ for $f, \sigma$ respectively, and write $h=h_{\text {top }}(f)=h_{\text {top }}(\sigma)$. Recall that $\Sigma$ has finitely many states. Also $(\Sigma, \sigma)$ is transitive, and if $f$ is topologically mixing then $(\Sigma, \sigma)$ is as well Bow70a, Proposition 30]. Since $\pi$ is finite-to-one (indeed, bounded-to-one), $\operatorname{Per}_{n}(f) \asymp \operatorname{Per}_{n}(\sigma)$. If $p \geq 1$ is the period of the TMS, then $\operatorname{Per}_{p n}(\sigma) \sim p e^{p n h}$, see e.g. Kit98, Observation 1.4.3]. Hence $\operatorname{Per}_{p n}(f) \asymp e^{p n h}$. If $f$ is topologically mixing, then $\operatorname{Per}_{n}(f) \asymp e^{n h}$.

Now let $f: M \rightarrow M$ be nonuniformly hyperbolic, where $M$ can have any dimension. Since $\pi$ is finite-to-one, there is a constant $C>0$ s.t. $\operatorname{Per}_{n}(f) \geq C \times \operatorname{Per}_{n}(\sigma)$ for all $n \geq 1$. The reverse inequality might not hold, because $\pi$ only codes trajectories inside $\mathrm{NUH}_{\chi}$, and $f$ can have many more (even uncountably many) periodic orbits outside of $\mathrm{NUH}_{\chi}$. Even if we only count isolated periodic orbits, the growth rate of $\operatorname{Per}_{n}(f)$ can be superexponential Kal00.

For a TMS with countably many states, good estimates on $\operatorname{Per}_{n}(\sigma)$ are related to the existence of measures of maximal entropy, as observed by Gurevic Gur69, Gur70. He showed that every transitive TMS admits at most one measure of maximal entropy, and such measure exists iff there is $p \geq 1$ s.t. for every vertex $v$ it holds

$$
\#\left\{\underline{v} \in \Sigma: \sigma^{p n}(\underline{v})=\underline{v}, v_{0}=v\right\} \asymp e^{p n h_{\max }},
$$

where $h_{\max }=h_{\max }(\sigma)=\sup \left\{h_{\nu}(\sigma): \nu\right.$ is $\sigma$-invariant probability measure on $\left.\Sigma\right\}$. We add the assumption that $f$ has a $\chi$-hyperbolic measure of maximal entropy, in which case $h=h_{\text {top }}(f)=h_{\max }(\sigma)$. Measures of maximal entropy exist for $C^{\infty}$ diffeomorphisms New89, although they might not be hyperbolic (e.g. the product of an Anosov diffeomorphism and an irrational rotation). For surface diffeomorphism with positive topological entropy, ergodic measures of maximal entropy are $\chi$-hyperbolic for every $\chi<h_{\text {top }}(f)$, by the Ruelle inequality.

Assuming that $f$ possesses a $\chi$-hyperbolic measure of maximal entropy, repeat the arguments used for uniformly hyperbolic systems inside a transitive component of $(\Sigma, \sigma)$. If $p \geq 1$ is the period of this component, then $\operatorname{Per}_{p n}(\sigma) \asymp e^{p n h}$, and so $\operatorname{Per}_{p n}(f) \geq C \times e^{p n h}$ for all $n \geq n_{0}$. This was proved by Sarig in dimension two Sar13, Theorem 1.1] and by Ben Ovadia in higher dimension [BO18, Theorem 1.4]. 
Let us make some comments on the period $p$. Assume that $f$ is topologically mixing. Recently, Buzzi was able to use the finite-to-one coding to define a oneto-one coding. Together with a precise counting on the TMS, he concluded that $\operatorname{Per}_{n}(f) \geq C \times e^{n h}$ for all $n \geq n_{0}$ Buz20. His proof works whenever the smooth system has a finite-to-one coding with some extra properties, which are true for diffeomorphisms [Sar13, BO18], and billiard maps LM18.

Now let $\varphi: M \rightarrow M$ be a flow and $\left(\Sigma_{r}, \sigma_{r}\right)$ be a TMF. Given $T>0$, let $\operatorname{Per}_{T}(\varphi)$ and $\operatorname{Per}_{T}\left(\sigma_{r}\right)$ denote the number of closed trajectories of $\varphi$ and $\sigma_{r}$, respectively, with minimal period $\leq T$. Note that $(\underline{v}, t) \in \operatorname{Per}_{T}\left(\sigma_{r}\right)$ iff there is a minimal period $n \geq 1$ s.t. $\underline{v} \in \operatorname{Per}_{n}(\sigma)$ and $r_{n}(\underline{v}) \leq T$, hence estimating $\operatorname{Per}_{T}\left(\sigma_{r}\right)$ is more complicated. For uniformly hyperbolic flows, there are precise estimates:

- Geodesic flows on closed hyperbolic surfaces: in constant curvature, Huber proved that $\operatorname{Per}_{T}(\varphi) \sim e^{T} / T$ Hub59. In variable curvature, Sinal gave the first estimates [Sin66], which were later significantly sharpened by Margulis [Mar69], who proved that $\operatorname{Per}_{T}(\varphi) \sim C e^{T h} / T$ where $C=1 / h$ (C. Toll, unpublished).

- Axiom A flows: Bowen proved $\operatorname{Per}_{T}(\varphi) \asymp e^{T h} / T$ Bow72a]. If the flow is topologically weak mixing, Parry and Pollicott proved that $\operatorname{Per}_{T}(\varphi) \sim e^{T h} / T h$ PP83, and Pollicott and Sharp found an estimate for the error term PS01.

We also mention a result for manifolds with Gromov hyperbolic fundamental group (e.g. manifolds that admit a metric with Anosov geodesic flow). Knieper and Coornaert counted free homotopy classes of closed geodesics estimating the growth rate of conjugacy classes in the fundamental group Kni83. CK02.

Now consider nonuniformly hyperbolic flows. For geodesic flows in nonpositively curved rank one manifolds, the following are known:

- Knieper showed that $\pi_{0}(T) \asymp e^{T h} / T$, where $\pi_{0}(T)$ counts the homotopy classes of simple closed geodesics with length less than $T$ Kni97, Kni02.

- For certain metrics constructed by Donnay Don88 and Burns and Gerber [BG89], $\operatorname{Per}_{T}(\varphi) \sim e^{T h} / T h$ Wea.

For the flows in Theorem 6.12 if there exists a measure of maximal entropy then there is $T_{0}>0$ s.t. $\operatorname{Per}_{T}(\varphi) \geq C \times e^{T h} / T$ for all $T \geq T_{0}$ LS19, Thm 8.1]. This estimate strengthens Katok's bound $\lim _{T \rightarrow \infty} \frac{1}{T} \log \operatorname{Per}_{T}(\varphi) \geq h$, see $\operatorname{Kat} 80$ Kat82. The proof in LS19 uses a dichotomy for TMF, see [LLS16, Theorem 4.6].

We end this section mentioning some results for two-dimensional billiard maps. As seen in Subsection 4.1. every billiard map preserves an invariant Liouville measure $\mu_{\mathrm{SRB}}$. Using the countable Markov partition constructed in [BSC90], Chernov proved that $\liminf \frac{1}{n} \log \operatorname{Per}_{n}(f) \geq h_{\mu_{\mathrm{SRB}}}(f)$ Che91, Corollary 2.4]. Better estimates can be obtained using measures of maximal entropy. Recently, Baladi and Demers gave sufficient conditions for periodic Lorentz gases (Sinaŭ billiards with non-intersecting scatterers) to have measures of maximal entropy BD20]. This occurs when the billiard map satisfies two properties:

(1) Finite horizon: there is no trajectory that makes only tangential collisions.

(2) $h_{*}>s_{0} \log 2$.

The second assumption requires some explanation. Part of their work consists on defining a topological entropy $h_{*}$ for finite horizon Lorentz gases, which is an upper bound for all metric entropies BD20, Thm 2.3(4)]. Fixing an angle $\theta_{0} \approx \frac{\pi}{2}$ and $n_{0}>0$, let $s_{0} \in(0,1)$ be the smallest number s.t. any orbit of length $n_{0}$ has at 
most $s_{0} n_{0}$ collisions with $|\theta|>\theta_{0}$. Under conditions (1)-(2), there is an $f$-adapted measure $\mu_{*}$ s.t. $h_{\mu_{*}}(f)=h_{*}$ BD20, Thm 2.4]. Using [LM18] and Buz20, it follows that $\operatorname{Per}_{n}(f) \geq C \times e^{n h_{*}}$ for $n$ sufficiently large.

Here are some examples of billiards satisfying the conditions (1)-(2) of BD20]. If $\mathcal{K}_{\text {min }}$ be the minimum curvature of the scatterer boundaries and $\tau_{\min }$ be the minimum free flight time, then $h_{*}>\log \left(1+2 \mathcal{K}_{\min } \tau_{\min }\right)$. Consider the two-parameter family $(r, R)$ of Lorentz gases in $\mathbb{T}^{2}$ with two discs as scatterers, one centered at the origin $(0,0)$ with radius $R$ and the other at $\left(\frac{1}{2}, \frac{1}{2}\right)$ with radius $r$, see figure 20. Baladi and Demers found a domain in the parameter space for which

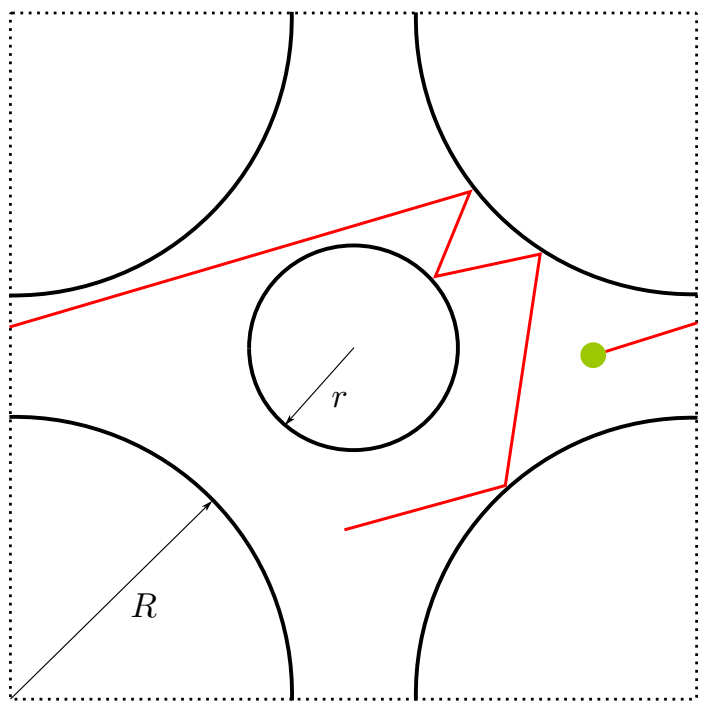

Figure 20. If $r, R$ are chosen inside a specific polygon in the parameter space then there is an $f$-adapted measure of maximal entropy, and $\operatorname{Per}_{n}(f) \geq C \times e^{n h_{*}}$ for all large $n$.

$\log \left(1+2 \mathcal{K}_{\min } \tau_{\min }\right) \geq \frac{1}{2} \log 2 \geq s_{0} \log 2$, hence $\mathrm{BD} 20$. Thm 2.4] applies. There are also numerical experiments dealing with scatterers located in a triangular lattice indicating that $h_{*}>s_{0} \log 2$ whenever the scatterers do not intersect and the billiard has finite horizon GB95.

\section{Equilibrium MEASURES}

Let $(Y, S)$, where $Y$ is a complete metric separable space and $S: Y \rightarrow Y$ is continuous, and let $\psi: Y \rightarrow \mathbb{R}$ be a continuous potential. The following definitions are standard.

Topological Pressure: The topological pressure of $\psi$ is $P_{\text {top }}(\psi):=\sup \left\{h_{\mu}(S)+\right.$ $\left.\int \psi d \mu\right\}$, where the supremum ranges over all $S$-invariant probability measures for which $\int \psi d \mu$ makes sense and $h_{\mu}(S)+\int \psi d \mu \neq \infty-\infty$.

EqUiLIBRIUM MEASURE: An equilibrium measure for $\psi$ is an $S$-invariant probability measure $\mu$ s.t. $P_{\text {top }}(\psi)=h_{\mu}(S)+\int \psi d \mu$.

A special case occurs when $\psi \equiv 0$ : equilibrium measures are measures of maximal entropy. If $\pi:(X, T) \rightarrow(Y, S)$ is finite-to-one, then equilibrium measures for $\psi$ lift 
to equilibrium measures for $\widehat{\psi}=\psi \circ \pi$. If $\pi$ is Hölder continuous, then $\widehat{\psi}$ is Hölder whenever $\psi$ is. In our context, we can apply the thermodynamical formalism for Hölder continuous potentials in TMS to obtain ergodic properties of equilibrium measures of Hölder continuous potential in uniformly and nonuniformly hyperbolic systems.

Since a transitive TMS with finitely many states has a unique measure of maximal entropy [Par64], then every uniformly hyperbolic transitive diffeomorphism has a unique measure of maximal entropy Bow70a, equal to the projection of the measure of maximal entropy in $(\Sigma, \sigma)$. Prior to this, Gurevič obtained some partial results, using the work of Sinar, and of Berg 10 Bowen also showed that every Hölder continuous potential has a unique equilibrium measure Bow74b, and it is either Bernoulli or Bernoulli times a period Bow74a.

Using the same analogy, Bowen and Ruelle proved that Hölder continuous potentials on uniformly hyperbolic flows have unique equilibrium measures BR75. In this case, equilibrium measures of $\left(\Sigma_{r}, \sigma_{r}\right)$ are related to equilibrium measures of $(\Sigma, \sigma)$, see BR75, Prop. 3.1].

For nonuniformly hyperbolic $C^{1+\beta}$ surface diffeomorphisms, Sarig proved that each Hölder continuous potential has at most countably many ergodic hyperbolic equilibrium measures Sar13, Thm 1.2], and each of them is either Bernoulli or Bernoulli times a period [Sar11]. The proof uses that for topologically transitive TMS each Hölder continuous potential has at most one equilibrium measure BS03], and different topologically transitive subgraphs of a TMS have disjoint vertex sets. The same holds for higher dimensional diffeomorphisms BO18, and for three dimensional flows LS19. In the flow case, each such equilibrium measure is either Bernoulli or Bernoulli times a rotation [LLS16]. Since geodesic flows cannot have rotational components (they are a particular case of Reeb flows), the following corollary holds: if $S$ is a closed smooth orientable Riemannian surface with nonpositive and non-identically zero curvature, then the geodesic flow of $S$ is Bernoulli with respect to its (unique) measure of maximal entropy, see [LLS16, Corollary 1.3].

Let us mention some results on the uniqueness of measures of maximal entropy for nonuniformly hyperbolic geodesic flows. The uniqueness referred in the previous paragraph follows from the work of Knieper, who proved it for geodesic flows on closed rank one manifolds Kni98, and also for geodesic flows on symmetric spaces of higher rank Kni05]. Gelfert and Ruggiero proved the uniqueness for geodesic flows on surfaces without focal points and genus greater than one GR19. Burns, Climenhaga, Fisher, and Thompson proved the uniqueness of many equilibrium states (including some multiples of the geometric potential and the zero potential) of geodesic flows on rank one manifolds [BCFT18], and there is a recent preprint that obtains similar results for geodesic flows on surfaces without focal points [CKP20]. There is also a recent preprint that proves the uniqueness of the measure of maximal entropy for geodesic flows on surfaces without conjugate points [CKW19].

Uniqueness of measures of maximal entropy for $C^{\infty}$ transitive surface diffeomorphisms with positive topological entropy has recently been obtained. Essentially, the results of Sar13 were not able to give uniqueness because it was not clear

\footnotetext{
${ }^{10}$ Berg proved that for hyperbolic toral automorphisms the Haar measure is the only measure of maximal entropy Ber69.
} 
how to overrule the possibility that two measures in the surface lift to two different transitive components of the TMS. This difficulty was solved by Buzzi, Crovisier, and Sarig BCS18], who showed that if two measures have positive entropy and are homoclinically related (a notion introduced in RHRHTU11) then they can be lifted to a same transitive component of the TMS. This can be regarded as a version of [Bow70a, Proposition 30]. They also prove that if the diffeomorphism is $C^{\infty}$, then all measures of maximal entropy are homoclinically related (this uses Yomdin's theory), hence there is a unique measure of maximal entropy. It would be interesting to obtain similar results for three dimensional flows and billiard maps.

\section{REFERENCES}

[Ad198] Roy L. Adler, Symbolic dynamics and Markov partitions, Bull. Amer. Math. Soc. (N.S.) 35 (1998), no. 1, 1-56.

[Ano67] D. V. Anosov, Tangential fields of transversal foliations in U-systems, Mat. Zametki 2 (1967), 539-548.

[Ano69] _ Geodesic flows on closed Riemann manifolds with negative curvature., Proceedings of the Steklov Institute of Mathematics, No. 90 (1967). Translated from the Russian by S. Feder, American Mathematical Society, Providence, R.I., 1969. MR0242194 (39 \#3527)

[Ano70] - On certain class of invariant sets of smooth dynamical systems (in Russian), Proc. 5th International Conference on Non-Linear Oscillations 2 (1970), 3945.

[AW67] R. L. Adler and B. Weiss, Entropy, a complete metric invariant for automorphisms of the torus, Proc. Nat. Acad. Sci. U.S.A. 57 (1967), 1573-1576. MR0212156 (35 \#3031)

[AW70] Roy L. Adler and Benjamin Weiss, Similarity of automorphisms of the torus, Memoirs of the American Mathematical Society, No. 98, American Mathematical Societ y, Providence, R.I., 1970. MR0257315 (41 \#1966)

[Bal95] Werner Ballmann, Lectures on spaces of nonpositive curvature, DMV Seminar, vol. 25, Birkhäuser Verlag, Basel, 1995. With an appendix by Misha Brin.

[BB17] Mike Boyle and Jérôme Buzzi, The almost Borel structure of surface diffeomorphisms, Markov shifts and their factors, J. Eur. Math. Soc. (JEMS) 19 (2017), no. 9, 2739-2782. MR3692886

[BCFT18] K. Burns, V. Climenhaga, T. Fisher, and D. J. Thompson, Unique equilibrium states for geodesic flows in nonpositive curvature, Geom. Funct. Anal. 28 (2018), no. 5, 1209-1259.

[BCL] J. Buzzi, S. Crovisier, and Y. Lima, $\chi$-universal Markov partitions for three dimensional flows. In preparation (2019).

[BCS18] Jérôme Buzzi, Sylvain Crovisier, and Omri Sarig, Measures of maximal entropy for surface diffeomorphisms, 2018. Preprint arXiv https://arxiv.org/abs/1811.02240

[BD04] Mike Boyle and Tomasz Downarowicz, The entropy theory of symbolic extensions, Invent. Math. 156 (2004), no. 1, 119-161.

[BD20] Viviane Baladi and Mark F. Demers, On the measure of maximal entropy for finite horizon Sinai Billiard maps, J. Amer. Math. Soc. 33 (2020), no. 2, 381-449.

[Ber68] Kenneth R. Berg, Entropy of torus automorphisms, Topological Dynamics (Symposium, Colorado State Univ., Ft. Collins, Colo., 1967), 1968, pp. 67-79.

[Ber69] _ Convolution of invariant measures, maximal entropy, Math. Systems Theory 3 (1969), 146-150.

[BG89] Keith Burns and Marlies Gerber, Real analytic Bernoulli geodesic flows on $S^{2}$, Ergodic Theory Dynam. Systems 9 (1989), no. 1, 27-45. MR991488 (90e:58126)

[BH11] Keith Burns and Boris Hasselblatt, The Sharkovsky theorem: a natural direct proof, Amer. Math. Monthly 118 (2011), no. 3, 229-244.

[BMW12] K. Burns, H. Masur, and A. Wilkinson, The Weil-Petersson geodesic flow is ergodic, Ann. of Math. (2) 175 (2012), no. 2, 835-908.

[BO18] Snir Ben Ovadia, Symbolic dynamics for non-uniformly hyperbolic diffeomorphisms of compact smooth manifolds, Journal of Modern Dynamics 13 (2018), 43-113. 
[BO19] _ Canonically codable points and irreducible codings, 2019. preprint arXiv:1904.01391.

[Bow08] Rufus Bowen, Equilibrium states and the ergodic theory of Anosov diffeomorphisms, revised, Lecture Notes in Mathematics, vol. 470, Springer-Verlag, Berlin, 2008. With a preface by David Ruelle, Edited by Jean-René Chazottes. MR2423393

[Bow17] _ Rufus Bowen's Notebook, 2017. Available at https://bowen.pims.math.ca/.

[Bow70a] , Markov partitions for Axiom A diffeomorphisms, Amer. J. Math. 92 (1970), 725-747. MR0277003 (43 \#2740)

[Bow70b] , Topological entropy and axiom A, Global Analysis (Proc. Sympos. Pure Math., Vol. XIV, Berkeley, Calif., 1968), 1970, pp. 23-41.

[Bow71] _ Periodic points and measures for Axiom A diffeomorphisms, Trans. Amer. Math. Soc. 154 (1971), 377-397.

[Bow72a] _ The equidistribution of closed geodesics, Amer. J. Math. 94 (1972), 413423. MR0315742 (47 \#4291)

[Bow72b] _ Periodic orbits for hyperbolic flows, Amer. J. Math. 94 (1972), 1-30.

[Bow73] _ Symbolic dynamics for hyperbolic flows, Amer. J. Math. 95 (1973), 429460. MR0339281 (49 \#4041)

[Bow74a] _ Bernoulli equilibrium states for Axiom A diffeomorphisms, Math. Systems Theory 8 (1974/75), no. 4, 289-294. MR0387539 (52 \#8379)

[Bow74b] Some systems with unique equilibrium states, Math. Systems Theory 8 (1974/75), no. 3, 193-202.

[Bow78a] _ Markov partitions are not smooth, Proc. Amer. Math. Soc. 71 (1978), no. 1, 130-132. MR0474415

[Bow78b] _ On Axiom A diffeomorphisms, American Mathematical Society, Providence, R.I., 1978. Regional Conference Series in Mathematics, No. 35 . MR0482842 (58 \#2888)

[BP07] Luis Barreira and Yakov Pesin, Nonuniform hyperbolicity, Encyclopedia of Mathematics and its Applications, vol. 115, Cambridge University Press, Cambridge, 2007. Dynamics of systems with nonzero Lyapunov exponents. MR2348606 (2010c:37067)

[BP13] _ Introduction to smooth ergodic theory, Graduate Studies in Mathematics, vol. 148, American Mathematical Society, Providence, RI, 2013.

[BPVL19] L. Backes, M. Poletti, P. Varandas, and Y. Lima, Simplicity of Lyapunov spectrum for linear cocycles over non-uniformly hyperbolic systems, Ergodic Theory Dynam. Systems (2019). Available online.

[BR75] Rufus Bowen and David Ruelle, The ergodic theory of Axiom A flows, Invent. Math. 29 (1975), no. 3, 181-202. MR0380889 (52 \#1786)

[BS02] Michael Brin and Garrett Stuck, Introduction to dynamical systems, Cambridge University Press, Cambridge, 2002.

[BS03] Jérôme Buzzi and Omri Sarig, Uniqueness of equilibrium measures for countable Markov shifts and multidimensional piecewise expanding maps, Ergodic Theory Dynam. Systems 23 (2003), no. 5, 1383-1400. MR2018604 (2004k:37053)

[BSC90] L. A. Bunimovich, Ya. G. Sină, and N. I. Chernov, Markov partitions for twodimensional hyperbolic billiards, Uspekhi Mat. Nauk 45 (1990), no. 3(273), 97134, 221.

[Bun74a] L. A. Bunimovič, Billiards that are close to scattering billiards, Mat. Sb. (N.S.) 94(136) (1974), 49-73, 159.

[Bun74b] _ The ergodic properties of certain billiards, Funkcional. Anal. i Priložen. 8 (1974), no. 3, 73-74.

[Bun79] L. A. Bunimovich, On the ergodic properties of nowhere dispersing billiards, Comm. Math. Phys. 65 (1979), no. 3, 295-312.

[Bur11] David Burguet, $C^{2}$ surface diffeomorphisms have symbolic extensions, Invent. Math. 186 (2011), no. 1, 191-236.

[Buz20] J. Buzzi, The degree of bowen factors and injective codings of diffeomorphisms, Journal of Modern Dynamics 16 (2020), 1-36.

[Che91] N. I. Chernov, Topological entropy and periodic points of two-dimensional hyperbolic billiards, Funktsional. Anal. i Prilozhen. 25 (1991), no. 1, 50-57. 
[CK02] M. Coornaert and G. Knieper, Growth of conjugacy classes in Gromov hyperbolic groups, Geom. Funct. Anal. 12 (2002), no. 3, 464-478.

[CKP20] D. Chen, L.-Y. Kao, and K. Park, Unique equilibrium states for geodesic flows over surfaces without focal points, Nonlinearity 33 (2020), no. 3, 1118-1155.

[CKW19] V. Climenhaga, G. Knieper, and K. War, Uniqueness of the measure of maximal entropy for geodesic flows on certain manifolds without conjugate points, 2019. arXiv preprint https://arxiv.org/abs/1903.09831.

[CM06] Nikolai Chernov and Roberto Markarian, Chaotic billiards, Mathematical Surveys and Monographs, vol. 127, American Mathematical Society, Providence, RI, 2006.

[Don88] Victor J. Donnay, Geodesic flow on the two-sphere. I. Positive measure entropy, Ergodic Theory Dynam. Systems 8 (1988), no. 4, 531-553. MR980796 (90e:58127a)

[Dow11] Tomasz Downarowicz, Entropy in dynamical systems, New Mathematical Monographs, vol. 18, Cambridge University Press, Cambridge, 2011.

[Dua94] Pedro Duarte, Plenty of elliptic islands for the standard family of area preserving maps, Ann. Inst. H. Poincaré Anal. Non Linéaire 11 (1994), no. 4, 359-409.

[Ebe01] Patrick Eberlein, Geodesic flows in manifolds of nonpositive curvature, Smooth ergodic theory and its applications (Seattle, WA, 1999), 2001, pp. 525-571.

[ELW11] M. Einsiedler, E. Lindenstrauss, and T. Ward, Entropy in dynamics, 2011. Available at http://maths.dur.ac.uk/ tpcc68/entropy/welcome.html.

[Fer99] Sébastien Ferenczi, Complexity of sequences and dynamical systems, Discrete Math. 206 (1999), no. 1-3, 145-154. Combinatorics and number theory (Tiruchirappalli, 1996).

[FHY83] A. Fathi, M.-R. Herman, and J.-C. Yoccoz, A proof of Pesin's stable manifold theorem, Geometric dynamics (Rio de Janeiro, 1981), 1983, pp. 177-215.

[Fil19] Simion Filip, Notes on the multiplicative ergodic theorem, Ergodic Theory Dynam. Systems 39 (2019), no. 5, 1153-1189.

[GB95] P. Gaspard and F. Baras, Chaotic scattering and diffusion in the Lorentz gas, Phys. Rev. E (3) 51 (1995), no. 6, part A, 5332-5352.

[GR19] Katrin Gelfert and Rafael O. Ruggiero, Geodesic flows modelled by expansive flows, Proc. Edinb. Math. Soc. (2) 62 (2019), no. 1, 61-95.

[Gur69] B. M. Gurevič, Topological entropy of a countable Markov chain, Dokl. Akad. Nauk SSSR 187 (1969), 715-718. MR0263162 (41 \#7767)

[Gur70] _ Shift entropy and Markov measures in the space of paths of a countable graph, Dokl. Akad. Nauk SSSR 192 (1970), 963-965. MR0268356 (42 \#3254)

[Had98] J. Hadamard, Sur la forme des lignes géodésiques à l'infini et sur les géodésiques des surfaces réglées du second ordre, Bull. Soc. Math. France 26 (1898), 195-216.

[Hed39] Gustav A. Hedlund, The dynamics of geodesic flows, Bull. Amer. Math. Soc. 45 (1939), no. 4, 241-260.

[Hof78] Franz Hofbauer, $\beta$-shifts have unique maximal measure, Monatsh. Math. 85 (1978), no. 3, 189-198. MR0492180

[Hof79] _ On intrinsic ergodicity of piecewise monotonic transformations with positive entropy, Israel J. Math. 34 (1979), no. 3, 213-237 (1980).

[Hof81] _ On intrinsic ergodicity of piecewise monotonic transformations with positive entropy. II., Israel J. Math. 38 (1981), no. 1-2, 107-115.

[Hop39] Eberhard Hopf, Statistik der geodätischen Linien in Mannigfaltigkeiten negativer Krümmung, Ber. Verh. Sächs. Akad. Wiss. Leipzig 91 (1939), 261-304.

[Hop40] - Statistik der Lösungen geodätischer Probleme vom unstabilen Typus. II, Math. Ann. 117 (1940), 590-608.

[Hub59] Heinz Huber, Zur analytischen Theorie hyperbolischen Raumformen und Bewegungsgruppen, Math. Ann. 138 (1959), 1-26. MR0109212 (22 \#99)

[Kal00] Vadim Yu. Kaloshin, Generic diffeomorphisms with superexponential growth of number of periodic orbits, Comm. Math. Phys. 211 (2000), no. 1, 253-271. MR1757015 (2001e:37035)

[Kat79] A. Katok, Bernoulli diffeomorphisms on surfaces, Ann. of Math. (2) 110 (1979), no. 3, 529-547. MR554383

[Kat80] _ Lyapunov exponents, entropy and periodic orbits for diffeomorphisms, Inst. Hautes Études Sci. Publ. Math. 51 (1980), 137-173. MR573822 (81i:28022) 
[Kat82] — Entropy and closed geodesics, Ergodic Theory Dynam. Systems 2 (1982), no. 3-4, 339-365 (1983). MR721728 (85b:53047)

[KH95] Anatole Katok and Boris Hasselblatt, Introduction to the modern theory of dynamical systems, Encyclopedia of Mathematics and its Applications, vol. 54, Cambridge University Press, Cambridge, 1995. With a supplementary chapter by Katok and Leonardo Mendoza. MR1326374 (96c:58055)

[Kit98] Bruce P. Kitchens, Symbolic dynamics, Universitext, Springer-Verlag, Berlin, 1998. One-sided, two-sided and countable state Markov shifts. MR1484730 (98k:58079)

[KM95] Anatole Katok and Leonardo Mendoza, Dynamical systems with non-uniformly hyperbolic behavior, Cambridge University Press, 1995. Supplement to "Introduction to the modern theory of dynamical systems".

[Kni02] Gerhard Knieper, Hyperbolic dynamics and Riemannian geometry, Handbook of dynamical systems, Vol. 1A, 2002, pp. 453-545. MR1928523 (2003i:37023)

[Kni05] _ The uniqueness of the maximal measure for geodesic flows on symmetric spaces of higher rank, Israel J. Math. 149 (2005), 171-183. Probability in mathematics.

[Kni83]__ Das Wachstum der Äquivalenzklassen geschlossener Geodätischer in kompakten Mannigfaltigkeiten, Arch. Math. (Basel) 40 (1983), no. 6, 559-568.

[Kni97] G. Knieper, On the asymptotic geometry of nonpositively curved manifolds, Geom. Funct. Anal. 7 (1997), no. 4, 755-782. MR1465601 (98h:53055)

[Kni98] Gerhard Knieper, The uniqueness of the measure of maximal entropy for geodesic flows on rank 1 manifolds, Ann. of Math. (2) 148 (1998), no. 1, 291-314. MR1652924 (2000b:37016)

[KSLP86] Anatole Katok, Jean-Marie Strelcyn, F. Ledrappier, and F. Przytycki, Invariant manifolds, entropy and billiards; smooth maps with singularities, Lecture Notes in Mathematics, vol. 1222, Springer-Verlag, Berlin, 1986.

[KU07] Svetlana Katok and Ilie Ugarcovici, Symbolic dynamics for the modular surface and beyond, Bull. Amer. Math. Soc. (N.S.) 44 (2007), no. 1, 87-132. MR2265011 (2007j:37050)

[LLS16] François Ledrappier, Yuri Lima, and Omri Sarig, Ergodic properties of equilibrium measures for smooth three dimensional flows, Comment. Math. Helv. 91 (2016), no. 1, 65-106. MR3471937

[LM18] Yuri Lima and Carlos Matheus, Symbolic dynamics for non-uniformly hyperbolic surface maps with discontinuities, Ann. Sci. Éc. Norm. Supér. (4) 51 (2018), no. 1, 1-38. MR3764037

[LS19] Yuri Lima and Omri M. Sarig, Symbolic dynamics for three-dimensional flows with positive topological entropy, J. Eur. Math. Soc. (JEMS) 21 (2019), no. 1, 199-256.

[Mar69] G. A. Margulis, Certain applications of ergodic theory to the investigation of manifolds of negative curvature, Funkcional. Anal. i Priložen. 3 (1969), no. 4, 89-90. MR0257933 (41 \#2582)

[Mor34] Marston Morse, Does instability imply transitivity?, Proceedings of the National Academy of Sciences of the United States of America 20 (1934), no. 1, 46-50.

[MT88] John Milnor and William Thurston, On iterated maps of the interval, Dynamical systems (College Park, MD, 1986-87), 1988, pp. 465-563. MR970571

[New89] Sheldon E. Newhouse, Continuity properties of entropy, Ann. of Math. (2) 129 (1989), no. 2, 215-235. MR986792 (90f:58108)

[Ose68] V. I. Oseledec, A multiplicative ergodic theorem. Characteristic Ljapunov, exponents of dynamical systems, Trudy Moskov. Mat. Obšč. 19 (1968), 179-210.

[Par64] William Parry, Intrinsic Markov chains, Trans. Amer. Math. Soc. 112 (1964), 5566. MR161372

[Pes76] Ja. B. Pesin, Families of invariant manifolds that correspond to nonzero characteristic exponents, Izv. Akad. Nauk SSSR Ser. Mat. 40 (1976), no. 6, 1332-1379, 1440. MR0458490 (56 \#16690)

[Pes77a] _ Characteristic Ljapunov exponents, and smooth ergodic theory, Uspehi Mat. Nauk 32 (1977), no. 4 (196), 55-112, 287. MR0466791 (57 \#6667)

[Pes77b] _ Geodesic flows in closed Riemannian manifolds without focal points, Izv. Akad. Nauk SSSR Ser. Mat. 41 (1977), no. 6, 1252-1288, 1447. MR0488169 
[PP83] William Parry and Mark Pollicott, An analogue of the prime number theorem for closed orbits of Axiom A flows, Ann. of Math. (2) 118 (1983), no. 3, 573-591. MR727704 (85i:58105)

[PS01] Mark Pollicott and Richard Sharp, Error terms for closed orbits of hyperbolic flows, Ergodic Theory Dynam. Systems 21 (2001), no. 2, 545-562. MR1827118 (2002f:37051)

[PT72] J. F. Plante and W. P. Thurston, Anosov flows and the fundamental group, Topology 11 (1972), 147-150.

[Rat69] M. E. Ratner, Markov decomposition for an U-flow on a three-dimensional manifold, Mat. Zametki 6 (1969), 693-704. MR0260977 (41 \#5597)

[Rat73] M. Ratner, Markov partitions for Anosov flows on n-dimensional manifolds, Israel J. Math. 15 (1973), 92-114. MR0339282 (49 \#4042)

[RHRHTU11] F. Rodriguez Hertz, M. A. Rodriguez Hertz, A. Tahzibi, and R. Ures, Uniqueness of SRB measures for transitive diffeomorphisms on surfaces, Comm. Math. Phys. 306 (2011), no. 1, 35-49.

[Sar11] Omri M. Sarig, Bernoulli equilibrium states for surface diffeomorphisms, J. Mod. Dyn. 5 (2011), no. 3, 593-608. MR2854097 (2012k:37071)

[Sar13] _ Symbolic dynamics for surface diffeomorphisms with positive entropy, J. Amer. Math. Soc. 26 (2013), no. 2, 341-426.

[Shu87] Michael Shub, Global stability of dynamical systems, Springer-Verlag, New York, 1987. With the collaboration of Albert Fathi and Rémi Langevin, Translated from the French by Joseph Christy. MR869255

[Sin66] Ja. G. Sină̌, Asymptotic behavior of closed geodesics on compact manifolds with negative curvature, Izv. Akad. Nauk SSSR Ser. Mat. 30 (1966), 1275-1296. MR0229178 (37 \#4752)

[Sin68a] — Construction of Markov partitionings, Funkcional. Anal. i Priložen. 2 (1968), no. 3, 70-80 (Loose errata).

[Sin68b] Markov partitions and U-diffeomorphisms, Funkcional. Anal. i Priložen 2 (1968), no. 1, 64-89.

[Sin70] Dynamical systems with elastic reflections. Ergodic properties of dispersing billiards, Uspehi Mat. Nauk 25 (1970), no. 2 (152), 141-192.

[Sma98] Steve Smale, Finding a horseshoe on the beaches of Rio, Math. Intelligencer 20 (1998), no. 1, 39-44.

[Tak73] Yōichirō Takahashi, Isomorphisms of $\beta$-automorphisms to Markov automorphisms, Osaka J. Math. 10 (1973), 175-184. MR0340552

[Wea] Bryce A. Weaver, Precise asymptotic growth rate of periodic orbits for a class of non-uniformly hyperbolic geodesic flows. preprint (2011).

[Yoc15] J.-C. Yoccoz, A proof of Jakobson's theorem, 2015. 34 pages, available at https://www.college-de-france.fr/media/jean-christopheyoccoz/UPL74162544747766 98194_Jakobson_jcy.pdf

[You98] Lai-Sang Young, Statistical properties of dynamical systems with some hyperbolicity, Ann. of Math. (2) 147 (1998), no. 3, 585-650.

Yuri Lima, Departamento de Matemática, Universidade Federal do Ceará (UfC), Campus do Pici, Bloco 914, CEP 60440-900. Fortaleza - CE, Brasil

E-mail address: yurilima@gmail.com 